\title{
INTEGRABILIDADE DE G-ESTRUTURAS
}

\author{
Gustavo Ignácio Duarte \\ DissERTAÇÃO APRESENTADA \\ $\mathrm{AO}$ \\ Instituto DE MATEMÁtica e Estatística \\ DA \\ UNIVERSIDADE DE SÃO PAULO \\ PARA \\ OBTENÇÃO DO TÍTULO \\ $\mathrm{DE}$ \\ Mestre eM CiênCIAS
}

Programa de Pós-Graduação em Matemática

Orientador: Prof. Dr. Ivan Struchiner

São Paulo, junho de 2018 


\section{INTEGRABILIDADE DE G-ESTRUTURAS}

Esta versão da dissertação contém as correções e alterações sugeridas pela Comissão Julgadora durante a defesa da versão original do trabalho, realizada em 28/05/2018. Uma cópia da versão original está disponível no

Instituto de Matemática e Estatística da Universidade de São Paulo.

Comissão Julgadora:

- Prof. Dr. Ivan Struchiner (orientador) - IME-USP

- Prof $^{\mathrm{a}}$. Dra ${ }^{\mathrm{a}}$.Maria Amelia Salazer Pinzón - UFF

- Prof. Dr. Lino Anderson da Silva Gama - Unicamp 


\section{Agradecimentos}

Meus agradecimentos vão para meus pais Teresa e Lourival, que me apoiam e me apoiarão por toda a vida, meu amigo Marcelo cuja amizade deu-me ânimo pra ir até o fim e ao amigo e orientador Ivan, que me deu a força necessária para concluir este trabalho. 


\section{Resumo}

DUARTE, G. I. Integrabilidade de G-estruturas. 2018. 102 f. Dissertação (Mestrado) - Instituto de Matemática e Estatística, Universidade de São Paulo, São Paulo, 2018.

Esta dissertação tem como objetivo discutir sob quais condições uma Gestrutura é integrável. Primeiro apresentam-se fibrados principais, vetoriais e outras estruturas a elas associados como torção, espaços verticais, espaços horizontais e conexões. Depois apresentam-se a definição de G-estrutura, de integrabilidade de G-estruturas, com exemplos e as respectivas versões de integrabilidade e equivalência de G-estruturas. Finalmente, são descritas condições mais gerais que garantem a integrabilidade de G-estruturas.

Palavras-chave: fibrados principais, fibrados vetoriais, fibrados associados, conexões, transporte paralelo, curvatura, torção, G-estruturas, integrabilidade, equivalência de G-estruturas, cohomologia de Spencer. 


\begin{abstract}
DUARTE, G.I. Integrability of G-structures. 2018. 102 f. Dissertation (Master) - Instituto de Matemática e Estatística, Universidade de São Paulo, São Paulo, 2018.

This dissertation aims to discuss what are the conditions for the integrability of a G-structure. We begin presenting principal bundles, vectoer bundles, associated bundles and other structures related to them like torsion, vertical spaces, horizontal spaces and connections. After this, we present the definition of G-structure, integrability os G-structures with examples ans respectives versions of integrabilities and the equivalence of G-estructures. Finally, we describe more general conditions that ensure the integrability of G-estrutures.
\end{abstract}

Keywords: principal bundles, vector bundles, associated bundles, connections, parallel transport, curvature, torsion, G-structures, integrability, equivalence of G-structures, Spencer cohomology. 


\section{Sumário}

1 Introdução 1

2 Fibrados Principais, Vetoriais e Associados. 3

2.1 Fibrados principais . . . . . . . . . . . . . . . 3

2.1 .1 Sobre as fibras . . . . . . . . . . . 5

2.1.2 Morfismos de fibrados principais . . . . . . . . 6

2.1 .3 Seções . . . . . . . . . . . . . . . . . . . 7

2.1.4 Exemplos . . . . . . . . . . . . . . 8

2.2 Fibrado associado . . . . . . . . . . . . . . . . 9

2.3 Fibrados vetoriais. . . . . . . . . . . . . . . . 13

2.3.1 Seções em fibrados associados. . . . . . . . . . . . 14

2.3.2 Pullback de fibrados vetoriais. . . . . . . . . . . . 16

2.3.3 Fibrados vetoriais como fibrados associados. . . . . . . 17

2.4 Conexões em fibrados principais. . . . . . . . . . . . . 18

2.4 .1 Espaço vertical. . . . . . . . . . . . . . . . 18

2.4.2 Conexões em fibrados principais e espaços horizontais. 20

2.4 .3 Formas de conexão. . . . . . . . . . . . . . . . . . . 21

2.4.4 Trasporte paralelo em fibrados principais . . . . . . 24

2.4.5 Curvatura. . . . . . . . . . . . . . 25

2.5 Conexões em fibrados vetoriais. . . . . . . . . . . . . . 28

2.5.1 Transporte paralelo em fibrados vetoriais. . . . . . . . 31

2.6 Curvatura e torção. . . . . . . . . . . . . . . . . . . . . 33

2.6 .1 Curvatura. . . . . . . . . . . . . 33

2.6 .2 Torção . . . . . . . . . . . . . . . . . . . 34

2.6.3 Quando a curvatura é zero. . . . . . . . . . . 35

2.6.4 Formas em fibrados associados . . . . . . . . . . 37

2.7 Conexões induzidas em fibrados associados . . . . . . . . 38 
2.8 Redução do grupo estrutural . . . . . . . . . . . . . . . 39

3 G-estruturas $\quad 41$

3.1 G-estruturas em variedades . . . . . . . . . . . . . . . . 41

3.1.1 Problema da equivalência de G-estruturas . . . . . . . 42

3.1 .2 Exemplos . . . . . . . . . . . . . . . 42

3.2 Conexões compatíveis . . . . . . . . . . . . . 55

3.2.1 Compatibilidade entre conexões e $G$-estruturas . . . . 55

3.2 .2 espaço das conexões compatíveis . . . . . . . . . 56

3.3 Curvatura e torção de G-estruturas . . . . . . . . . . . . . 57

3.3 .1 Torção intrínseca . . . . . . . . . . . . . . . 57

3.4 Equações estruturais, curvatura e torção . . . . . . . . . . . . 59

3.4.1 Forma tautológica . . . . . . . . . . . . . . . 59

3.4.2 Torção e equações estruturais. . . . . . . . . . . . . . 61

4 Integração de G-estruturas $\quad 69$

4.1 Prolongamentos e Cohomologia de Spencer . . . . . . . . . 69

4.2 Contato de k-ésima ordem e jatos. . . . . . . . . . . . 71

4.3 Fibrados referenciais e forma tautológica . . . . . . . 72

4.4 Funções estruturais . . . . . . . . . . . . . . . 75

4.5 G-estruturas uniformemente $k$-integráveis $\ldots \ldots \ldots \ldots$

4.6 G-estruturas de tipo finita . . . . . . . . . . . . 78

$\begin{array}{ll}\text { A Apêndice } & 79\end{array}$

A.1 Variedades: quocientes . . . . . . . . . . . . . . . . 79

A.2 Cálculos de Cartan . . . . . . . . . . . . . . . . . 80

A.2.1 Derivada de Lie de um campo vetorial . . . . . . 80

A.2.2 Contração ou produto interior . . . . . . . . . . . . . 81

A.2.3 Derivadas de Lie em formas diferenciais . . . . . . . . 82

A.3 Conexão de Levi-Civita . . . . . . . . . . . . . . . . . . . . 84

A.4 Lema ou Truque de Moser Local . . . . . . . . . . . . 86

A.5 Propriedade Universal de Maurer-Cartan . . . . . . . . . 88

A.6 Exemplo não-integrável: variedades nearly Kähler . . . . . 89

A.7 $G_{2}$-estruturas . . . . . . . . . . . . . . 90

$\begin{array}{ll}\text { Referências Bibliográficas } & 91\end{array}$

Índice Remissivo $\quad 93$ 


\section{Capítulo 1}

\section{Introdução}

As variedades são objetos centrais no estudo da Geometria. Muitas de suas propriedades podem ser estudadas a partir de um espaço vetorial muito importante: os espaços tangentes.

E podemos definir estruturas nos espaços tangentes que nos dão muitas informações importantes sobre a geometria da variedade (comprimento, volume etc.): produtos internos , orientações, folheações, estruturas simpléticas etc.

Existem certos referenciais (bases) dos espaços tangentes que são compatíveis com a estrutura em questão. Por exemplo, os referenciais ortonormais mantém a a informação produto interno, ou equivalentemente o conjunto das bases ortonormais para o produto interno.

A ação de certas transformações lineares sobre esses referenciais, que são elementos de subgrupos de Lie de $G L_{n}\left(\mathbb{R}^{n}\right)$ mantém invariante as propriedades geométricas em relação à estrutura. No mesmo exemplo, as transformações ortogonais, que pertencem ao grupo $O(n)$ representado pelas matrizes ortogonais, conservam informação do o produto interno, ou de forma equivalente o conjunto de bases ortonormais para o produto interno.

Dessa forma, obtemos um conjunto de referenciais especiais que tem uma compatibilidade com a estrutura em questão codificada pelo subgrupo de Lie que o mantém invariante. As G-estruturas são exatamente tais conjuntos de referenciais que através da escolha de $G \subseteq G L_{n}\left(\mathbb{R}^{n}\right)$, subgrupo de Lie, que determina um tipo de geometria.

Assim, essa forma de enxergar a Geometria, através do estudo das Gestruturas, permite um estudo dela mais global e com uma sistematização mais geral.

Uma G-estrutura é integrável se em torno de cada ponto da variedade existem cartas para os quais o referencial induzido funciona como acima descrito. Com isso, estudaremos teoremas clássicos de integrabilidade como os caso Riemanniano, em que a integrabilidade está relaciona com a curvatura e torção, o teorema de Frobenius, em que a integrabilidade está relacionada 
com a involutividade de uma distribuição, teorema de Darboux que trata da integrabilidade de formas simpléticas.

Uma grande questão é a equivalência de G-estruturas: em que condições localmente existe um isomorfismo entre entre duas G-estruturas, cujo caso particular é a equivalência a chamada G-estrutura canônica ou standard, de $\mathbb{R}^{n}$.

O objetivo desse trabalho é encontrar tais condições e isso é desenvolvido no artigo The Integrability Problem for G-Structures, de Victor Guillemin ([Gui65]), com uma linguagem mais moderna que terá como base a linguagem de fibrados e trará uma série de ferramentas que serão muito úteis ao nosso propósito. 


\section{Capítulo 2}

\section{Fibrados Principais, Vetoriais e Associados.}

Começaremos apresentando espaços de projeção: fibrados.

Iniciamos com o fibrado principal que é a estrutura básica na construção de G-estruturas. Em seguida estudaremos os fibrados associados que são o elo entre os fibrados principais e os fibrados vetoriais, última estrutura estudada nesse capítulo.

Em seguida veremos os conceitos de conexão e curvatura primeiro em fibrados principais e depois em fibrados vetoriais. Na primeira situação, serão apesentados os conceitos de espaços verticais e horizontais e suas propriedades geométricas. Também veremos as 1-formas de conexão e sua relação com espaços horizontais. Depois estudaremos conexões e curvatura em fibrados vetoriais,e como elas se correlacionam. Além disso, estudaremos o transporte paralelo em ambos fibrados.

Esse capítulo tem como forte suporte [Cra15]

\subsection{Fibrados principais}

Definição 2.1.1. Sejam $M$ uma variedade diferenciável e $G$ um grupo de Lie. Um G-fibrado principal sobre $M$ consiste de

(i) uma variedade $P$ e uma ação à direita $P \times G \rightarrow P,(p, g) \mapsto p g$ de $G$ sobre P.

(ii) uma submersão sobrejetora $\pi: P \rightarrow M G$-invariante, isto é, $\pi(p g)=$ 
$\pi(p)$, denominada projeção, que satisfaz a propriedade de trivialidade local: dado $x_{0} \in M$ existem uma vizinhança aberta desse ponto $U$ e um difeomorfismo

$$
\Psi_{U}: \pi^{-1}(U) \rightarrow U \times G
$$

que leva cada fibra $\pi^{-1}(x)$ nas fibras $\{x\} \times G$ e é $G$-equivariante: $\Psi_{U}(p g)=$ $\Psi_{U}(p) g$, pela ação $G$ dada por: $(x, g) h=(x, g h), h \in G$.

$P$ é chamado de espaço total, $M$ é de espaço base. Usaremos as notações $P_{x}$ para a fibra $\pi^{-1}(x)$ e $\left.P\right|_{U}$ para $\pi^{-1}(U)$.

Exemplo 2.1.1. $O$ G-fibrado principais trivial sobre $M$ é $M \times G$, munido com a ação descrita acima e a projeção como sendo a projeção $p_{1}: M \times G \rightarrow$ $M, p_{1}(x, g)=x$.

Proposição 2.1.1. A ação de $G$ sobre Pé livre, própria e transitiva nas fibras.

Demonstração. Primeiro vamos provar que ação á direita de $G$ sobre $U \times G$ é livre: $(x, a) g=(x, a) \Rightarrow(x, a g)=(x, a) \Rightarrow a g=a$ e multiplicando ambos os membros da ultima igualdade por $a^{-1}$, obtemos $g=e$.

Agora, suponhamos que $p \cdot g=p$, com $p \in P, g \in G$. Aplicando uma trivialização local $\Psi$ nesta igualdade, tem-se

$$
\Psi(p g)=\Psi(g) \Rightarrow \Psi(p) g=\Psi(p) \Rightarrow g=e
$$

Portanto, a ação de $G$ sobre $P$ é livre.

Para provar que a ação é própria, temos que provar que a função

$$
P \times G \rightarrow P \times P,(p, g) \mapsto(p, p g)
$$

é própria.

Uma aplicação é própria se a pré-imagem de um compacto no contradomínio é um compacto. O que equivale a provar que essa pré-imagem é sequencialmente compacta em nosso caso.

Seja $K \subseteq P \times P$, compacto, e $\left(\left(p_{i}, g_{i}\right)\right)_{i}$ uma sequência em $P \times G$, tal que $\left(\left(p_{i}, p_{i} g_{i}\right)\right) \subseteq K$, para todo $i$. Nesse caso, com esta sequência está num compacto, ela admite uma subsequência convergente $\left(\left(p_{i}, p_{i} g_{i}\right)\right)_{i}$, cujo limite será $(p, q) \in K$. Consideremos a subsequência $\left(\left(p_{i}, g_{i}\right)\right)_{i}$ que é levada na subsequência convergente. 
Seja $x=\pi(P)$ e $U \subseteq M$ um abeto trivializante em torno de M, cuja trivialização local é $\psi: E_{x} \longrightarrow U \times G$. Sabemos que:

$$
p_{i} \rightarrow p \Rightarrow \pi\left(p_{i}\right) \rightarrow \pi(p)
$$

e por isso, exceto por um número finito de índices, $\pi\left(p_{i}\right) \in U$.

Além disso,

$$
\begin{aligned}
p_{i} g_{i} \rightarrow q & \Rightarrow \pi\left(p_{i} g_{i}\right)=\pi\left(p_{i}\right) \rightarrow \pi(q) \\
& \Rightarrow \pi(p)=\pi(p) \\
& \therefore q \in E_{x}
\end{aligned}
$$

Sejam $\psi(p)=(x, a), \psi(q)=(x, b)$ e $\psi\left(p_{i}\right)=\left(x_{i}, a_{i}\right)$. Então $x_{\rightarrow} x, a_{i} \rightarrow a \mathrm{e}$ como $p_{i} g_{i} \rightarrow q$, então

$$
\begin{aligned}
\psi\left(p_{i} g_{i}\right) \rightarrow \psi(q) & \Rightarrow \psi\left(p_{i}\right) g_{i} \rightarrow(x, b) \\
& \left(x_{i}, a_{i} g_{i}\right) \rightarrow(x, b) \\
& \Rightarrow a_{i} g_{i} \rightarrow b
\end{aligned}
$$

Logo,

$$
a_{i} g_{i} \rightarrow b \Rightarrow g_{i} \rightarrow a_{i}^{-1} b
$$

Finalmente, $\left(p_{i}, g_{i}\right) \rightarrow\left(p, a_{i}^{-1} b\right)$

\subsubsection{Sobre as fibras}

Como $\pi$ é uma função G-invariante, as fibras $\pi^{-1}(x)$ são G-espaços difeomorfos ao próprio grupo $\mathrm{G}$ (como G-espaços): fixado $x \in M$ todo $p \in \pi^{-1}(x)$ induz um difeomorfismo

$$
\begin{gathered}
\phi: G \rightarrow P_{x} \\
\phi_{p}(g)=p g, \quad p \in P_{x}
\end{gathered}
$$

compatível coma a ação à direita de de G: $\phi(g h)=\phi(g) h, h \in G$

Denotando por $g_{p \rightarrow q}$ o único elemento em $G$ tal que $q=p g$ temos a inversa da aplicação acima:

$$
\phi_{p}^{-1}: P_{x} \rightarrow G, \phi_{p}^{-1}(q)=g_{p \rightarrow q}
$$


Observação 1. Consideremos $P \times{ }_{M} P=\{(p, q): \pi(p)=\pi(q)\}$. Dessa forma,conseguimos um difeomorfismo

$$
\begin{gathered}
\delta: P \times G \rightarrow P \times_{M} P \\
(p, g) \mapsto(p, p g)
\end{gathered}
$$

cuja inversa é

$$
\begin{gathered}
\Delta: P \times_{M} P \rightarrow P \times G \\
(p, q) \mapsto\left(p, g_{p \rightarrow q}\right)
\end{gathered}
$$

\subsubsection{Morfismos de fibrados principais}

Definição 2.1.2. Se $\pi_{i}: P_{i} \rightarrow M, i=1,2$ são G-fibrados principais, um morfismo entre G-fibrados principais é um uma função suave $F: P_{1} \rightarrow$ $P_{2}$ que comuta com as projeções: $\pi_{1}=\pi_{2} \circ F$ e é G-equivariante: $F(p g)=$ $F(p) g, p \in P_{1}, g \in G$.

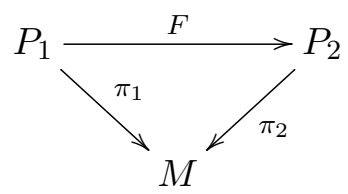

Um G-fibrado principal é dito trivializável se é isomorfo ao fibrado principal trivial.

A condição de trivialidade local afirma que, $\pi^{-1}(U)$ é isomorfo ao fibrado principal trivial.

Proposição 2.1.2. Todo morfismo entre fibrados principais é isomorfismo.

Demonstração. Sejam $\pi_{1}: P_{1} \rightarrow M$ e $\pi_{2}: P_{2} \rightarrow M$ dois G-fibrados principais e $f: P_{1} \rightarrow P_{2}$ um morfismo entre eles. Ou seja, $\pi_{2} \circ f=\pi_{1}$.

Sejam $p, q \in P_{1}$ tais que $f(p)=f(q)$. Então

$$
\pi_{2}(f(p))=\pi_{2}(f(q)) \Rightarrow \pi_{1}(p)=\pi_{1}(q)
$$

Desse modo, $p$ e $q$ pertencem à mesma fibra $\pi^{-1}(x)$, com $x \in M$. Como a ação de $G$ é transitiva, existe $g \in G$ tal que $q=p g$. Daí

$$
f(q)=f(p g)=f(p) g=f(q) g
$$


Como a ação é livre, então $g=e$. Portanto, $q=p$. E assim, $f$ é injetora.

Agora, consideremos $\bar{p} \in P_{2}$. Tomemos $b \in \pi_{2}(\bar{p})$. Vamos tomar agora $p \in \pi_{1}^{-1}(b)$. Sabemos que $\bar{p} \in \pi_{2}^{-1}(b)$ também.

Então, existe $g \in G$ tal que

$$
\bar{p}=f(p) g \Rightarrow \bar{p}=f(p g)
$$

Logo, $f$ é sobrejetora.

Seja $h: P_{2} \rightarrow P_{1}$ a inversa de $f$. Vamos provar que $g$ é G-equivariante. Seja $q \in P_{2}$ e $g \in G$. Então $q=f(p), p \in P_{1}$ de modo que $q=g(p)$. Assim:

$$
h(q g)=h(f(p) g)=h(f(p g))=p g=h(q) g
$$

A função $g$ também é continua: consideremos as respectivas trivializações locais $\Psi: P_{i}: \pi_{i}^{-1}(U) \rightarrow U \times G, i=1,2$, para $U \subseteq M$ aberto. Então, podemos considerar $\left.f\right|_{U}: U \times G \rightarrow U \times G$ com

$$
\left.f\right|_{U}(x, g)=(x, \rho(x, g))
$$

com $\rho: U \times G \rightarrow G$, tal que $\rho(x, h g)=\rho(x, h) \cdot g$. Como $f$ é contínua, então $\rho$ também o é. Além disso, como $\rho(x, g) \in G$, existe $\rho^{-1}(x, g)$ e a aplicação $g \mapsto g^{-1}$ é contínua em grupos topológicos, que é o caso de $G$ um grupo de Lie.

Assim, $\left.f\right|_{U}$ é localmente expressa por $(x, g) \mapsto\left(x, \rho^{-1}(g)\right)$.

\subsubsection{Seções}

Definição 2.1.3. Se $\pi: P \rightarrow M$ é um G-fibrado principal, uma seção desse fibrado será um função $\sigma: M \rightarrow P$ em que $\sigma(x) \in \pi^{-1}(x)$, ou seja, $\pi_{P} \circ \sigma=I d_{M}$.

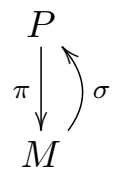

O espaço da seções de um fibrado principal é denotado por por $\Gamma_{M}(P)$ ou simplesmente $\Gamma(P)$ se não houver dúvidas quanto ao espaço base. 
Proposição 2.1.3. Um G-fibrado principal admite seção global se, e somente se,é trivializável.

Demonstração. Se o fibrado principal é trivializável, então existe um isomorfismo $f: M \times G \rightarrow P$. Vamos definir, para $g \in G$ fixado, a seguinte função:

$$
\sigma_{g}: M \rightarrow P, \sigma_{g}(x)=f(x, g)
$$

Isto define uma seção pois $f$ leva fibra em fibra de modo que $f(x, g) \in$ $\pi^{-1}(x)$.

Se $\sigma$ é uma seção global do G-fibrado principal $\pi: P \rightarrow M$ então

$$
F_{\sigma}: M \times G \rightarrow P, F_{\sigma}(x, g)=\sigma(x) g
$$

é um isomorfismo de G-fibrados principais. Considerando as projeções $p_{1}: M \times G \rightarrow M$ a projeção na primeira coordenada e $\pi: P \rightarrow M$ então:

$$
\begin{gathered}
\pi \circ F_{\sigma}(x, g)=\pi(\sigma(x) g)=\pi(\sigma(x))=x \\
p_{1}(x, g)=x
\end{gathered}
$$

Portanto, $p_{1}=\pi \circ F_{\sigma}$. Assim, $F_{\sigma}$ é um isomorfismo de G-fibrados principais, de modo que $P$ é trivializável.

\subsubsection{Exemplos}

Alguns exemplos de G-fibrados principais:

1. Seja $\mathbb{Z}_{2}=\{e, a\}$ com $a^{2}=e$ e definamos a ação desse grupo sobre $S^{n}$ da seguinte maneira:

$$
z e=z, z a=-z, \quad z \in S^{n}
$$

O quociente $S^{n} / \mathbb{Z}_{2}$ é difeomorfo ao espaço projetivo $\mathbb{R} P^{n}$, de modo a obtermos o $\mathbb{Z}_{2}$-fibrado principal $\pi: S^{n} \rightarrow \mathbb{R} P^{n}$.

2. Seja $G=S^{1}$, identificado com $\{z \in \mathbb{C}:|z|=1\}$. Definindo a ação de 
$S^{1}$ sobre $S O(3)$ como sendo

$$
M z=M\left[\begin{array}{ccc}
1 & 0 & 0 \\
0 & \cos \theta & \sin \theta \\
0 & -\sin \theta & \cos \theta
\end{array}\right], M \in S O(3), \quad z=\cos \theta+i \sin \theta
$$

e a projeção como sendo $\pi: S O(3) \rightarrow S^{2}$ que associa $M \in S O(3)$ à sua primeira coluna.

Então $\pi: S O(3) \rightarrow S^{2}$ é um $S^{1}$ - fibrado principal.

3. Considere $S^{3}=\left\{\left(z_{1}, z_{2}\right) \in \mathbb{C}^{2}:\left|z_{1}\right|^{2}+\left|z_{2}\right|^{2}=1\right\}$ e a ação de $S^{1}$ sobre $S^{3}$ dada por

$$
\left(z_{1}, z_{2}\right) \cdot z=\left(z_{1} z, z_{2} z\right), \quad z \in S^{1}
$$

e a projeção

$$
\pi: S^{3} \rightarrow S^{2}, \pi\left(z_{1}, z_{2}\right)=\left(2 z_{1} \bar{z}_{2},\left|z_{1}\right|^{2}-\left|z_{2}\right|^{2}\right)
$$

$\pi: S^{3} \rightarrow S^{2}$ é um $S^{1}$ - fibrado principal. Este é a chamada fibração de Hopf.

4. Sejam $G$ um grupo de Lie e $H$ um subgrupo de Lie fechado e do primeiro.

A ação de $H$ sobre $G$ é dada por multiplicação. Então $\pi: G \rightarrow$ $G / H, \pi(g)=[g]$ é um $H$-fibrado principal, cujas fibras são exatamente as co-classes à esquerda de $G / H$.

\section{$2.2 \quad$ Fibrado associado}

Sejam $F$ uma variedade, $G \times F \rightarrow F$ uma ação de $G$ à esquerda sobre $F$ e $\pi: P \rightarrow M$ um G-fibrado principal.

Definição 2.2.1. O fibrado associado ao fibrado principal é definido como

$$
E(P, F)=(P \times F) / G
$$

onde $(p, v) \sim\left(p g, g^{-1} v\right)$. 
Definimos a seguinte projeção

$$
\begin{gathered}
\tilde{\pi}: E(P, F) \rightarrow M \\
\tilde{\pi}([p, v])=\pi(p)
\end{gathered}
$$

Denotando por $[p, v]$ os elementos induzidos por $(p, v)$, observemos que

$$
[p g, v]=\left[p g, g^{-1} g v\right]=[p, g v]
$$

Quanto às fibras,

$$
\tilde{\pi}^{-1}(x)=E(P, V)_{x}=\left\{[p, v] \in E(P, V): p \in \pi^{-1}(x), v \in F\right\}
$$

Dado $p_{0} \in \pi^{-1}(x)$, temos

$$
\begin{aligned}
p_{0} \in \pi^{-1}(x) & \Rightarrow p_{0}=p g, g \in G \\
& \Rightarrow\left[p_{0}, v\right]=[p g, v]=[p, g v]=[p, w]
\end{aligned}
$$

Assim, $\pi^{-1}(x)=\left\{\left[p_{0}, v\right]: v \in F\right\}$

Proposição 2.2.1. Seja $E(P, F)$ um fibrado associado. Então:

1. $E(P, F)$ é uma variedade.

2. $\tilde{\pi}: E(P, F) \rightarrow M$ é uma submersão sobrejetora.

3. Em torno de cada $x \in M$ existe um aberto $U \subseteq M$ tal que existe um difeomorfismo

$$
\tilde{\Psi}_{U}:\left.E(P, F)\right|_{U} \rightarrow E \times F
$$

tal que o diagrama

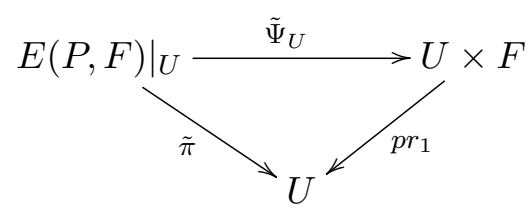

comuta 
4. Para todos abertos $U$ e $V$ trivializantes, com $U \cap V \neq \varnothing$ e $\left.\tilde{\Psi}\right|_{U}$ e $\left.\tilde{\Psi}\right|_{V}$ as respectivas trivializações, então

$$
\begin{gathered}
\left.\left.\tilde{\Psi}\right|_{V} \circ \tilde{\Psi}\right|_{U} ^{-1}:(U \cap V) \times F \rightarrow(U \cap V) \times F \\
\left.\left.\tilde{\Psi}\right|_{V} \circ \tilde{\Psi}\right|_{U} ^{-1}(x, v)=\left(x, g_{U V}(\sigma(x)) v\right)
\end{gathered}
$$

com

$$
g_{U V}: U \cap V \rightarrow G
$$

difeomorfismo. Além disso, se $U \cap V \cap W \neq \varnothing$, tem-se

$$
g_{V W} \circ g_{U V}=g_{U W}
$$

Demonstração. 1. Vamos considerar $\left\{U_{\alpha}\right\}$ uma cobertura de abertos trivializantes de $M$.

Consideremos uma seção local $\sigma_{\alpha}:\left.U_{\alpha} \longrightarrow P\right|_{U_{\alpha}}$.

Para cada $p \in P \mid U_{\alpha}$, existe um único elemento $g_{\alpha}(p) \in G$ de modo que $p=\sigma_{\alpha}(\pi(p)) g_{\alpha}(p)$. Isso define uma aplicação suave $g_{\alpha}: P \mid U_{\alpha} \longrightarrow G$.

A partir de $g_{\alpha}$, vamos definir a seguinte trivialização local

$$
\begin{gathered}
\tilde{\Psi}_{\alpha}:\left.E(P, V)\right|_{U_{\alpha}} \longrightarrow U_{\alpha} \times F \\
{[p, v] \mapsto\left(\pi(p), g_{\alpha}(p) v\right) .}
\end{gathered}
$$

A sua inversa é dada por $(x, v)=\left[\sigma_{\alpha}(x), v\right]$.

2. O diagrama a seguir

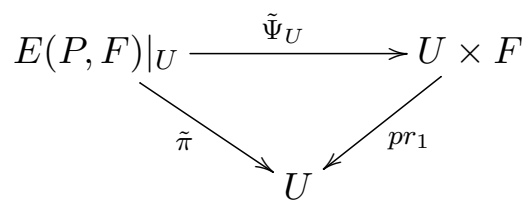

comuta por construção. Desse modo,

$$
p r_{1} \circ \tilde{\psi}=\tilde{\pi}
$$

de forma que a ultima função é, localmente, a composição de duas funções sobrejetora, e portanto, sobrejetora. Além disso, novamente 
localmente, $\tilde{\pi}$ expressa-se como uma projeção, de modo a ser uma submersão.

3. Provado em (1).

4. Seja $x \in U \cap V \neq \varnothing, U$ e $V$ abertos trivializantes de $M$, cujas respectivas trivializações associadas são $\tilde{\Psi}_{U}$ e $\tilde{\Psi}_{V}$ e as respectivas trivializações locais e $\sigma_{U V}: U \cap V \longrightarrow P_{U \cap V}$ uma seção. Além disso, seja $g_{U V}: U \cap V \rightarrow G$ como em (1). Se $\left.p \in P\right|_{U \cap V}$, então

$$
\begin{aligned}
\tilde{\Psi}_{U}^{-1}(x, v) & =\left[\sigma_{U}(x), v\right] \Rightarrow \\
\tilde{\Psi}_{V}\left(\tilde{\Psi}_{U}^{-1}(x, v)\right) & =\left(\pi\left(\sigma_{U}(x)\right), g_{U V}\left(\sigma_{U V}(x)\right) v\right) \\
& \left.=\left(x, g_{U V}\left(\sigma_{U V}(x)\right)\right) v\right)
\end{aligned}
$$

Sejam agora $U, V$ e $W$ abertos trivializantes de $\pi: E \rightarrow P$, tais que $U \cap$ $V \cap W \neq \varnothing$, com respectivas trivializações $\left.\tilde{\Psi}\right|_{U},\left.\tilde{\Psi}\right|_{V}$ e $\left.\tilde{\Psi}\right|_{W}$. Ademais, sejam $g_{U V}: U \cap V \rightarrow G, g_{V W}: V \cap W \rightarrow G, g_{U W}: U \cap W \rightarrow G$ como em (1) e $\sigma_{U V W}:\left.U \cap V \cap W \longrightarrow P\right|_{U \cap V \cap W}$. Então:

$$
\left.\left.\tilde{\Psi}\right|_{W} \circ \tilde{\Psi}\right|_{U} ^{-1}=\left.\left.\left.\left.\tilde{\Psi}\right|_{W} \circ \tilde{\Psi}\right|_{V} ^{-1} \circ \tilde{\Psi}\right|_{V} \circ \tilde{\Psi}\right|_{U} ^{-1}
$$

Se $x \in U \cap V \cap W$ e $v \in F$, então

$$
\left.\left.\left.\tilde{\Psi}\right|_{W} \circ \tilde{\Psi}\right|_{U} ^{-1}(x, v)=\left(x, g_{U W}\left(\sigma_{U V W}(x)\right)\right) v\right)
$$

e

$$
\begin{aligned}
\left.\left.\left.\left.\tilde{\Psi}\right|_{W} \circ \tilde{\Psi}\right|_{V} ^{-1} \circ \tilde{\Psi}\right|_{V} \circ \tilde{\Psi}\right|_{U} ^{-1}(x, v) & \left.=\left.\left.\tilde{\Psi}\right|_{W} \circ \tilde{\Psi}\right|_{V} ^{-1}\left(x, g_{U V}\left(\sigma_{U V W}(x)\right)\right) v\right) \\
& =\left.\tilde{\Psi}\right|_{W}\left(\left[\sigma_{U V W}(x), g_{U V}\left(\sigma_{U V W}(x) v\right)\right]\right) \\
& =\left(x, g_{V W} g_{U V}\left(\sigma_{U V W}(x) v\right)\right)
\end{aligned}
$$

de modo que $g_{U W}=g_{V W} g_{U V}$

Exemplo 2.2.1. Quando $F=V$ é um espaço vetorial e $\rho: G \rightarrow G L(V)$ é 
uma representação de $G$ em $V$, temos o seguinte fibrado associado:

$$
E(P, V)=(P \times V) / G
$$

onde ação de $G$ sobre $P \times V$ é dada por $(p, v) g=\left(p g, \rho^{-1}(g) v\right)$, em que denotaremos $\rho^{-1}(g) v$ simplesmente por $g^{-1} v$. Neste caso o fibrado associado é um fibrado vetorial.

Observação 2. Um espaço $E$ com projeção $\pi: E \longrightarrow M$ satisfazendo 1-4 é chamado fibrado com fibra $\boldsymbol{F}$ e grupo de estrutura $\boldsymbol{G}$.

\subsection{Fibrados vetoriais.}

Podemos assim definir as operações de espaço vetorial para o fibrado associado:

$$
[p, u]+[p, v]=[p, u+v] \text { e } \alpha[p, v]=[p, \alpha v], \alpha \in \mathbb{R}
$$

Observação 3. Considerando elementos arbitrários $[p, u]$ e $[q, v]$, existe $g \in G$ tal que $p=q \cdot g$, de modo que

$$
[q, v]=[p, g v]
$$

e assim

$$
[p, u]+[q, v]=[p, u]+[p, g v]=[p, u+g v] .
$$

Proposição 2.3.1. A operação de soma acima definida está bem definida.

Demonstração. Sejam $p$ e $q$ elementos da mesma fibra $\pi^{-1}(x)$. Então $p=$ $q \cdot g . g \in G$. Sejam também $(p, u) \sim\left(p^{\prime}, u^{\prime}\right)$ e $(q, v) \sim\left(q^{\prime}, v^{\prime}\right)$. Assim,

$$
(p, u) \cdot g_{1}=\left(p^{\prime}, u^{\prime}\right),(q, v) \cdot g_{2}=\left(q^{\prime}, v^{\prime}\right), g_{1}, g_{2} \in G
$$

Portanto,

$$
\begin{aligned}
& \left(p^{\prime}, u^{\prime}\right)=\left(p g_{1}, g_{1} u\right) \\
& \left(q^{\prime}, v^{\prime}\right)=\left(q g_{2}, g_{2} v\right)
\end{aligned}
$$


Desse modo,

$$
\begin{aligned}
{\left[p^{\prime}, u^{\prime}\right]+\left[q^{\prime}, v^{\prime}\right] } & =\left[p g_{1}, g_{1}^{-1} u\right]+\left[q g_{2}, g_{2}^{-1} v\right] \\
& =\left[p, g_{1} g_{1}^{-1} u\right]+\left[q, g_{2} g_{2}^{-1} u\right] \\
& =[p, u]+[q, v]
\end{aligned}
$$

Seja $x \in M$ e escolhamos um elemento $p \in P$.

Proposição 2.3.2. A função

$$
\begin{gathered}
\varphi_{p}: V \rightarrow E(P, V)_{x} \\
\varphi_{p}(v)=[p, v]
\end{gathered}
$$

é um isomorfismo de espaços vetoriais:

Demonstração. Decorre diretamente da proposição anterior.

\subsubsection{Seções em fibrados associados.}

Seja $E(P, F)$ um fibrado associado.

Consideremos o espaço das funções suaves equivariantes $C^{\infty}(P, F)^{G}=$ $\left\{f \in C^{\infty}(P, F): f(p \cdot g)=g^{-1} \cdot f(p)\right\}$.

Proposição 2.3.3. A função: $\gamma: C^{\infty}(P, F)^{G} \rightarrow \Gamma(E(P, F)), \gamma(f)(x)=$ $[p, f(p)]$, onde $p \in P x$, é uma bijeção.

Demonstração. A função $\gamma$ está bem definida: seja $q \in P_{x}$. Então $q=p g$. Assim

$$
\begin{aligned}
{[q, f(q)] } & =[p g, f(p g)] \\
& =\left[p g, g^{-1} f(p)\right] \\
& =[p, f(g)]
\end{aligned}
$$

Provemos que $\gamma$ é injetora:

$\gamma(f)(p)=\gamma\left(f^{\prime}\right)(p) \Rightarrow[p, f(p)]=\left[p, f^{\prime}(p)\right] \Rightarrow f(p)=f^{\prime}(p), \forall p \in P \Rightarrow f^{\prime}=f$ 
Provemos que $\gamma$ é sobrejetora: seja $\sigma \in \Gamma(E(P, F))$. Então $\sigma(x)=\left[p_{0}, v_{0}\right]$, $p_{0} \in P_{x}$.

Seja $q \in P_{x}$, de modo que $q=p_{0} g$. Assim

$$
\begin{aligned}
{\left[p_{0}, v_{0}\right] } & =\left[q g^{-1}, v_{0}\right] \\
& =\left[q, g^{-1} v_{0}\right]
\end{aligned}
$$

Definindo $f_{\sigma}(q)=g^{-1} v_{0}$, obtemos $\sigma(x)=\left[q, f_{\sigma}(v)\right]=\gamma(f)(x)$.

Definição 2.3.1. Seja Muma variedade. Um fibrado vetorial de posto $k$ sobre $M$ consiste de de uma sobrejeção $\pi: E \rightarrow M$, em que E é uma variedade e em torno de cada $x_{0} \in M$ pode-se encontrar um aberto $U$ e um isomorfismo para o qual seguinte diagrama comuta

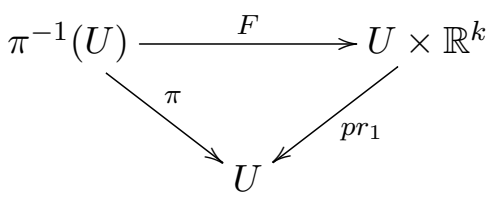

o que induz estrutura de espaço vetorial nas fibras.

Exemplo 2.3.1. O fibrado vetorial $p_{1}: M \times \mathbb{R}^{k} \rightarrow M, p_{1}(x, v)=x$ é o chamado fibrado trivial.

Definição 2.3.2. Dado um fibrado vetorial $\pi: E \rightarrow M$ uma seção é uma função suave $s: M \rightarrow E$ tal que $\pi \circ s=I d_{M}$, ou seja, $s(x) \in E_{x}$. O conjunto das seções de um fibrado vetorial é denotado por $\Gamma(E)$.

Exemplo 2.3.2. O fibrado tangente $\pi: T M \rightarrow M$, onde $T M=\bigcup_{x \in M} T_{p} M$ $e \pi(x, v)=x \in M$ é um fibrado vetorial. As fibras $\pi^{-1}(x)$ são exatamente os espaços tangentes $T_{p} M$. Se $(U, \phi)=\left(U,\left(x_{1}, \ldots, x_{n}\right)\right)$ é uma carta local para $M$,com $\phi(U) \subseteq \mathbb{R}^{n}$ então definimos a seguinte função:

$$
\begin{aligned}
\Phi_{U}: \pi^{-1}(U) & \rightarrow U \times \mathbb{R}^{n} \\
\Phi_{U}\left(x, \sum_{i=1}^{n} v_{1} \frac{\partial}{\partial x_{i}}\right) & =\left(x,\left(v_{1}, \ldots, v_{n}\right)\right)
\end{aligned}
$$

esta função é um difeomorfismo e, portanto, uma trivialização local de TM. 
Se restringirmos $\Phi_{U}$ ao espaço tangente $T_{p} M$, obtemos:

$$
\begin{aligned}
\left.\Phi_{U}\right|_{T_{x} M}: T_{p} M & \rightarrow\{x\} \times \mathbb{R}^{n} \\
\Phi_{U}\left(\sum_{i=1}^{n} v_{1} \frac{\partial}{\partial x_{i}}\right) & =\left(v_{1}, \ldots, v_{n}\right)
\end{aligned}
$$

é um isomorfismo linear, de modo que cumprem-se as condições de fibrado vetorial para o fibrado tangente.

Definição 2.3.3. Um referencial em $x \in M$ é uma coleção de seções $\left\{s_{1}, \ldots, s_{k}\right\}$ de $E$ tais que $\left\{s_{1}(x), \ldots, s_{k}(x)\right\}$ é base de $E_{x}$.

Dada $s \in \Gamma(E)$ e $\left\{s_{1}, \ldots, s_{k}\right\}$ um referencial em $x \in U$ aberto em $M$, então podemos expressá-la como:

$$
s(x)=f_{1}(x) s_{1}(x)+\ldots+f_{k}(x) s_{k}(x)
$$

em que $f_{i}$ são funções suaves em $U$.

\subsubsection{Pullback de fibrados vetoriais.}

Sejam $\pi: E \rightarrow N$ um fibrados vetorial de posto $k$ e $f \in C^{\infty}(M, N)$.

Definição 2.3.4. O fibrado pullback de $\pi: E \rightarrow N$ por $f$, denotado por $f^{*} E$, é definido como:

$$
\left(f^{*} E\right)=\{(x, p) \in M \times E: f(x)=\pi(p)\}
$$

com a projeção $\operatorname{pr}_{1}: f^{*} E \rightarrow M$.

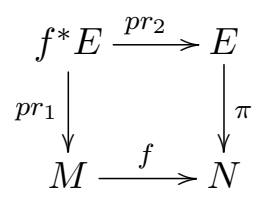

Seja $V \subseteq N$ um aberto em torno de $y=f(x)$, trivializante. Então existe $\psi: E_{V} \rightarrow V \times \mathbb{R}^{k}$ uma trivialização local do fibrado $\pi: E \rightarrow N$, com $\psi(p)=(\pi(p), \eta(p)), \eta: E_{\pi(p)} \rightarrow \mathbb{R}^{k}$, difeomorfismo nas fibras.

Consideremos

$$
\bar{\psi}: p r_{2}^{-1}\left(f^{-1}(V)\right) \rightarrow f^{-1}(V), \bar{\psi}(x, p)=(x, \eta(p))
$$


Estas são trivializações locais do fibrado pullback.

Observação 4. Em termos de fibras, $\left(f^{*} E\right)_{x} \simeq E_{f}(x)$.

Observação 5. As seções em $E$ induzem seções em $f^{*} E$ da seguinte maneira:

$$
\left(f^{*} s\right)(x)=s(f(x))
$$

Se $s \in \Gamma(E)$ então $f^{*} s=s \circ f: M \rightarrow E$ é de fato uma seção pois $\left(f^{*} s\right)(x)=s(f(x)) \in E_{f(x)}=\left(f^{*} E\right)_{x}$.

Lema 2.3.1. Seja $\alpha \in \Gamma\left(f^{*} E\right)$ e $U$ um aberto em $M$ em torno de $x$. Então $\alpha(x)=\sum_{i=1}^{k} u_{i}(x)\left(f^{*} s_{i}\right)(x)$, onde $\left\{s_{i}\right\}_{i=1}^{k}$ é um referencial de $x$ e $u_{i} \in$ $C^{\infty}(U)$.

Demonstração. Seja $\alpha \in \Gamma\left(f^{*} E\right)$, Então $\alpha(x) \in\left(f^{*} E\right)_{x}=E_{f(x)} \operatorname{com} x \in M$. Seja também $x \in M$ com $\left\{s_{i}\right\}_{i=1}^{k}$ um referencial em torno de $f(x)$. Dessa forma, existem $u_{1}, \ldots, u_{k} \in C^{\infty}(U)$ tais que

$$
\begin{aligned}
\alpha(x) & =\sum_{i=1}^{k} u_{i} s_{i}(f(x)) \\
& =\sum_{i=1}^{k} u_{i}\left(f^{*} s_{i}(x)\right)
\end{aligned}
$$

\subsubsection{Fibrados vetoriais como fibrados associados.}

\section{Fibrado referencial.}

Seja $\pi: E \rightarrow M$ é um fibrado vetorial de posto $k$. Vimos que um referencial $\left\{s_{i}\right\}_{i=1}^{k}$ em $x \in M$ é uma base de $E_{x}$, que pode ser visto como um isomorfismo

$$
\begin{gathered}
\phi: \mathbb{R}^{k} \rightarrow E_{x} \\
e_{i} \mapsto s_{i}(x)
\end{gathered}
$$

em que $\left\{e_{1}, \ldots, e_{k}\right\}$ é a base de canônica de $\mathbb{R}^{k}$.

Se $g \in G L_{k}(\mathbb{R})$ então $\phi \circ g: \mathbb{R}^{k} \rightarrow E_{x}$ continua sendo um isomorfismo e assim $\phi \circ g$ também é um referencial. 
Seja $\operatorname{Fr}(E)=\left\{(x, \phi): \phi\right.$ é um referencial de $\left.E_{x}\right\}$, o chamado fibrado referencial.

Definindo esta composição como uma ação à direita de $G L_{k}(\mathbb{R})$ sobre $F r(E)$, então $\bar{\pi}: F(E) \rightarrow M$ com $\bar{\pi}=p r_{1}$, é um $G L_{k}(\mathbb{R})$-fibrado principal, cujas fibras sobre $x$ são $F(E)_{x}=\left\{\phi: \mathbb{R}^{n} \rightarrow E_{x}\right.$ referencial $\}$

Seja $x \in M$ e $U$ um aberto em torno de $x$ de modo que existe $\psi_{U}: E_{U} \rightarrow$ $U \times \mathbb{R}^{k}$ uma trivialização local do fibrado. Então $\left.\psi_{U}\right|_{E_{x}}: E_{x} \rightarrow\{x\} \times \mathbb{R}^{k} \cong \mathbb{R}^{k}$ é um isomorfismo de maneira a ser um referencial.

Dessa forma, $\psi_{U} \circ \phi \in G L_{k}(\mathbb{R})$. A função:

$$
\begin{gathered}
\Psi_{U}:\left.\operatorname{Fr}(E)\right|_{U} \rightarrow U \times G L_{k}(\mathbb{R}) \\
(x, \phi) \mapsto\left(x, \psi_{U} \circ \phi\right)
\end{gathered}
$$

Quando $E=T M$, denotamos $\operatorname{Fr}(T M)$ por, simplesmente, $\operatorname{Fr}(M)$.

Proposição 2.3.4. Todo fibrado vetorial é um fibrado associado para algum fibrado principal.

Demonstração. Seja $\pi: E \rightarrow M$ um fibrado vetorial de posto $k$. Então o fibrado referencial $p r_{1}: \operatorname{Fr}(E) \rightarrow M$ é um $G L_{k}(\mathbb{R})$-fibrado principal.

Então $E\left(F r(E), \mathbb{R}^{n}\right)$ é o fibrado associado ao fibrado referencial, aplicação

$$
\begin{gathered}
f: E\left(F r(E), \mathbb{R}^{k}\right) \rightarrow E \\
{[\phi, v] \mapsto \phi(v)}
\end{gathered}
$$

é um isomorfismo entre $E$ e o fibrado associado.

\subsection{Conexões em fibrados principais.}

\subsubsection{Espaço vertical.}

Seja $\pi: P \rightarrow M$ um G-fibrado principal e a sua diferencial em $p \in P$, $(d \pi)_{p}: T_{p} P \rightarrow T_{\pi(p)} M$.

Definição 2.4.1. O espaço vertical em $p$ é definido por

$$
V_{p}=k e r(d \pi)_{p} \subseteq T_{p} P
$$


Observação 6. Se $x=\pi(p)$, os elementos do espaço vertical $V_{p}$ são exatamente os vetores tangentes à fibra $P_{x}$, ou seja $V_{p}=T_{p}\left(P_{x}\right)$.

Proposição 2.4.1. Existe um isomorfismo entre $\mathfrak{g}$, a álgebra de Lie de $G$, $e V_{p}$.

Demonstração. Vamos definir a ação infinitesimal de $\mathfrak{g}$ em $P$ :

$$
\begin{gathered}
\psi: \mathfrak{g} \rightarrow \mathfrak{X}(P) \\
\psi_{p}(\xi)=\left.\frac{d}{d t}\right|_{t=0} p \exp (t \xi)
\end{gathered}
$$

A correspondência $p \mapsto \psi_{p}(\xi)$ é o chamado campo vetorial fundamental gerado por $\xi$

Observemos que $\psi_{P}(\xi)$ é vertical:

$$
\begin{aligned}
\pi_{*}\left(\left.\frac{d}{d t}\right|_{t=0} p \exp (t \xi)\right) & \left.=\left.\frac{d}{d t}\right|_{t=0} \pi(\underbrace{\exp (t \xi)}_{\in G})\right) \\
& =\left.\frac{d}{d t}\right|_{t=0} \pi(p) \\
& =0
\end{aligned}
$$

Então, a imagem da aplicação $\psi$ está contida em $V_{p}$. Seja $p \in \pi^{-1}(x)$.

A aplicação

$$
\begin{gathered}
\phi_{p}: G \rightarrow P_{x} \\
g \mapsto p g
\end{gathered}
$$

é um difeomorfismo tal que $d \phi_{p}=\psi_{p}$. Observando o diagrama a seguir, com $\delta$ e $\Delta$ como em 2.2 e 2.3 , respectivamente.

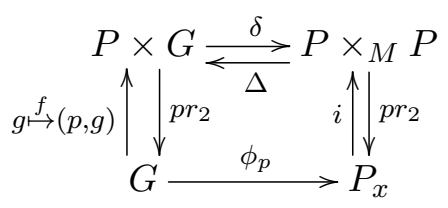

vemos que $\phi_{p}$ é um suave pois

$$
\phi_{p}=p r_{2} \circ \delta \circ f
$$

que é uma composição de funções suaves e sua inversa é $p r_{2} \circ \Delta \circ i$ que é 
suave pelo mesmo motivo. Assim, $\phi_{p}$ é um difeomorfismo. Dese modo, sua diferencial em $p$, que é $\psi_{p}$, é um isomorfismo linear. Desse modo,

$$
\mathfrak{g} \simeq V_{p}
$$

\subsubsection{Conexões em fibrados principais e espaços horizontais.}

Definição 2.4.2. Uma conexão em um G-fibrado principal é uma distribuição em $P$, ou seja, um subfibrado $H \subseteq T P$ em que

(a) $T_{p} P=V_{p} \oplus H_{p}$

(b) $\left(d R_{g}\right)_{p}\left(H_{p}\right)=H_{p g}, g \in G$

O espaço $H_{p}$ é chamado de espaço horizontal.

Os elementos de $V_{p}$ são chamados de vetores verticais e os elementos de $H_{p}$ de vetores horizontais.

Proposição 2.4.2. A restrição $\left.(d \pi)_{p}\right|_{H_{p}}: H_{p} \rightarrow T_{\pi(p)} M$ é um isomorfismo.

Demonstração. Temos que $\left.(d \pi)\right|_{H_{p}}$ é injetora pois $k e r\left(d \pi_{p}\right)=V_{p}$ e $V_{P} \cap H_{p}=\{0\}$. Além disso,

$$
\begin{gathered}
\operatorname{dim} T_{p} P=\operatorname{dim}\left(\operatorname{ker}(d \pi)_{p}\right)+\operatorname{dim}\left(\operatorname{Im}(d \pi)_{p}\right)=\operatorname{dim} V_{p}+\operatorname{dim} T_{\pi(q)} M \\
\operatorname{dim}_{p} P=\operatorname{dim} V_{p}+\operatorname{dim} H_{p}
\end{gathered}
$$

Desse modo

$$
\operatorname{dim} H_{p}=\operatorname{dim} T_{\pi(p)} M
$$

Proposição 2.4.3. Se $H_{p}$ é um espaço horizontal, existe um função

$$
h_{p}: T_{q} M \rightarrow T_{p} P
$$

chamada de levantamento horizontal tal que

$$
(d \pi)_{p} \circ h_{p}=I d
$$

e cuja imagem é $H_{p}$ 
Demonstração. Como $\left.(d \pi)_{p}\right|_{H_{p}}: H_{p} \rightarrow T_{\pi(p)} M$ é um isomorfismo, existe sua inversa $h: T_{\pi(p)} M \rightarrow H_{p}$.

A função $h_{p}$ obtém-se justamente quando se estende o contradomínio de $h$ até $T_{p} P$.

\subsubsection{Formas de conexão.}

Definição 2.4.3. Uma 1-forma de conexão em $P$ é uma 1-forma $\omega \in$ $\Omega^{1}(P, \mathfrak{g})$ tal que:

1. $\omega_{p}\left(\psi_{p}(\xi)\right)=\xi$.

2. $R_{g}^{*}(\omega)=A d_{g^{-1}}(\omega)$

Exemplo 2.4.1 (Retirado de [Dup03]). Considere o G-fibrado principal trivial $\pi: M \times G \rightarrow M, \pi(x, g)=p r_{1}(x, g)=x$.

Vamos definir

$$
\begin{gathered}
\omega_{M C}: T_{(p, g)} M \times G \rightarrow T_{e} G=\mathfrak{g} \\
\omega_{M C}=\left(L_{g^{-1}} \circ p r_{2}\right)_{*}
\end{gathered}
$$

onde $\mathrm{pr}_{2}: M \times G \rightarrow G$, com $\mathrm{pr}_{2}(x, g)=g$ e $L_{g^{-1}}: G \rightarrow G$ em que $L_{g^{-1}}(h)=g^{-1} h$. Esta é a chamada forma de Maurer-Cartan.

Observemos que

$$
\begin{aligned}
\omega_{M C} & =\left(L_{g^{-1}} \circ p r_{2}\right)_{*} \\
& =\left(L_{g^{-1}}\right)_{*} \circ\left(p r_{2}\right)_{*} \\
& =\left(p r_{2}\right)^{*}\left(\left(L_{g^{-1}}\right)_{*}\right) \\
& =\left(p r_{2}\right)^{*} \theta, \theta_{g}=\left(L_{g^{-1}}\right)_{*}
\end{aligned}
$$

Além disso, $R_{g}^{*} \omega_{M C}=R_{g}^{*} \circ p r_{2}^{*} \theta=p r_{2}^{*} \circ R_{g}^{*} \theta$. Vamos provara a segunda condição da definição para $\theta$ :

$$
\begin{aligned}
R_{g}^{*}(\theta)_{h} & =\theta_{h g} \circ\left(R_{g}\right)_{*}=\left(L_{(h g)^{-1}}\right)_{*} \circ\left(R_{g}\right)_{*} \\
& =\left(L_{g^{-1}}\right)_{*}\left(L_{h^{-1}}\right)_{*} \circ\left(R_{g}\right)_{*}=A d g^{-1}\left(L_{h^{-1}}\right)_{*} \\
& =A d g^{-1} \theta_{g}
\end{aligned}
$$


Lema 2.4.1. Sejam $\pi: P \rightarrow M$ e $\pi^{\prime}: P^{\prime} \rightarrow M^{\prime}$ dois G-fibrados principais e $f: P \rightarrow P^{\prime}$ um difeomorfismo. Se $\omega^{\prime}$ é uma 1-forma de conexão em $P^{\prime}$, então $f^{*} \omega^{\prime}$ é uma 1-forma de conexão em $P$.

Demonstração. Vamos mostrar que $f^{*} \omega^{\prime}$ é de fato uma 1-forma de conexão.

1. Como $\omega^{\prime}$ é 1-forma de conexão então: $\omega_{f(p)}^{\prime}\left(\psi_{f(p)}(v)\right)=v$. Dessa forma:

$$
\begin{aligned}
\left(f^{*} \omega^{\prime}\right)_{p}\left(\psi_{p}(v)\right) & =\omega_{f(p)}^{\prime}\left(d f_{p}\left(\psi_{p}(v)\right)\right) \\
& =\omega_{f(p)}^{\prime}\left(\psi_{f(p)}(v)\right) \\
& =v
\end{aligned}
$$

2. $R_{g}^{*}\left(\omega^{\prime}\right)=A d_{g^{-1}}\left(\omega^{\prime}\right)$. Então, se $X_{p} \in T_{p} P$,

$$
\begin{aligned}
R_{g}^{*}\left(\omega_{f(p)}^{\prime}\right)\left(d f_{p}\left(X_{p}\right)\right) & =A d_{g^{-1}}\left(\omega^{\prime}\right)\left(d f_{p}\left(X_{p}\right)\right) \Rightarrow \\
R_{g}^{*}\left(f^{*} \omega^{\prime}\right)_{p}\left(\left(X_{p}\right)\right) & =A d_{g^{-1}}\left(f^{*} \omega^{\prime}\right)_{p}\left(X_{p}\right), \forall X_{p} \in T_{p} P \Rightarrow \\
R_{g}^{*}\left(f^{*} \omega^{\prime}\right) & =A d_{g^{-1}}\left(f^{*} \omega^{\prime}\right)
\end{aligned}
$$

Proposição 2.4.4. Todo G-fibrado principal admite uma 1-forma de conexão.

Demonstração. (Baseado em [Dup03])

Seja $\left\{U_{\lambda}\right\}$ uma cobertura de abertos trivializantes de $M$ e sejam $\Psi_{\lambda}$ as respectivas trivializações. Pelo exemplo 1.4.1, existe uma 1-forma de conexão $\omega_{\lambda}$ em $U_{\lambda} \times G$. Então, pelo lema, $\Psi_{\lambda}^{*} \omega_{\lambda}$ é uma conexão em $\left.E\right|_{U_{\lambda}}$.

Seja $\left\{\rho_{\lambda}\right\}$ uma partição da unidade subordinada à cobertura. Definamos:

$$
\omega=\sum_{\lambda} \rho_{\lambda} \Psi_{\lambda}^{*} \omega_{\lambda}
$$

Provemos que $\omega$ é uma 1-forma de conexão:

1. $\omega_{p}\left(\psi_{p}(v)\right)=\sum_{\lambda} \rho_{\lambda}(p)\left(\Psi_{\lambda}^{*}\left(\omega_{\lambda}\right)_{p}\left(\psi_{p}(v)\right)=\sum_{\lambda} \rho_{\lambda}(p)(v)=v\right.$ 
2.

$$
\begin{aligned}
R_{g}^{*}(\omega) & =R_{g}^{*}\left(\sum_{\lambda} \rho_{\lambda} \Psi_{\lambda}^{*} \omega_{\lambda}\right)=\sum_{\lambda} \rho_{\lambda} \Psi_{\lambda}^{*} \omega_{\lambda}\left(\left(R_{g}\right)_{*}\right) \\
& =\sum_{\lambda} \rho_{\lambda} R_{g}^{*}\left(\Psi_{\lambda}^{*} \omega_{\lambda}\right)=\sum_{\lambda} \rho_{\lambda}\left(A d_{g^{-1}}\left(\Psi_{\lambda}^{*} \omega_{\lambda}\right)\right) \\
& =\left(A d_{g^{-1}}\left(\sum_{\lambda} \rho_{\lambda} \Psi_{\lambda}^{*} \omega_{\lambda}\right)\right)=A d_{g^{-1}}(\omega)
\end{aligned}
$$

Proposição 2.4.5. Existe uma correspondência 1-1 entre conexões $H \subseteq$ $T P$ e 1-formas de conexão $\omega \in \Omega(P,(g))$.

Demonstração. Seja $H$ uma conexão. Se $X \in \mathfrak{X}(P)$ então $X_{p}=X_{p}^{V}+X_{p}^{H}$ $\operatorname{com} X_{P}^{V} \in V_{p}$ e e $X_{P}^{H} \in H_{p}$. Definamos $\omega^{H} \in \Omega^{1}(P, \mathfrak{g})$ tal que

$$
\omega_{p}^{H}\left(X_{P}\right)=\psi_{p}^{-1}\left(X_{p}^{V}\right) \in \mathfrak{g}
$$

Essa é uma forma 1-forma de conexão pois:

1. $\omega_{p}^{H}\left(\psi_{p}(\xi)\right)=\psi_{p}^{-1}\left(\psi_{p}(\xi)\right)=\xi$

2. Se $X$ é horizontal, vale a condição (2) da definição. Se $X$ é vertical, então $X=\psi_{p}(\xi), \xi \in \mathfrak{g} \mathrm{e}$

$$
\begin{aligned}
\left.\left(R_{g}\right)_{p}^{*}\left(\omega_{p}^{H}(X)\right)\right) & =\omega_{p g}^{H}\left(\left(R_{g}\right)_{*}\left(\psi_{p}(\xi)\right)\right) \\
& =\omega_{p g}^{H}\left(\psi_{p g}\left(A d_{g^{-1}} \xi\right)\right) \\
& =A d_{g^{-1}}(\xi) \\
& =A d_{g^{-1}} \omega\left(\psi_{p}(\xi)\right) \\
& =A d_{g^{-1}}(\omega(X))
\end{aligned}
$$

Por outro lado, se $\omega \in \Omega^{1}(P, \mathfrak{g})$ é uma 1-forma de conexão então:

$$
\operatorname{ker}\left(\omega_{p}\right)=\left\{X_{P} \in T_{p} P: \omega\left(X_{p}\right)=0\right\}
$$

é um espaço horizontal.

Sabemos que $\omega_{p}: T_{p} P \rightarrow \mathfrak{g}$ é linear. Além disso, pela condição 1 da definição de 1-forma de conexão, temo que $V_{p} \subseteq \operatorname{Im}(\omega)_{p} \subseteq \mathfrak{g}$. Como $\mathfrak{g} \simeq V_{p}$ 
(Proposição 1.4.1) então $\operatorname{Im}\left(\omega_{p}\right)=\mathfrak{g}$. Desse modo

$$
\begin{aligned}
\operatorname{dim}\left(\operatorname{ker}\left(\omega_{p}\right)\right) & =\operatorname{dim} T_{p} P-\operatorname{dim} \mathfrak{g} \\
& =\operatorname{dim}_{p} P-V_{p}
\end{aligned}
$$

Seja $X_{p} \in k e r\left(\omega_{p}\right)$. Então:

$$
\omega_{p g}\left(\left(R_{g}\right)_{*} \omega\right)\left(X_{p}\right)=\left(\left(R_{g}\right)^{*} \omega_{p}\right)\left(X_{p}\right)=A d_{g^{-1}}\left(\omega\left(X_{p}\right)\right)=0
$$

Portanto $\left(d R_{g}\right)_{p}\left(X_{p}\right) \in H_{p g}=k e r\left(\omega_{p g}\right)$

Partindo de uma conexão $H$ e construindo a correspondente 1-forma de conexão $\omega$ come descrita acima, então $X_{p} \in \operatorname{ker}\left(\omega_{p}\right)$ se $\omega\left(X_{p}\right)=0$. Mas isso ocorre se, e somente se, $\psi_{p}^{-1}\left(X_{p}^{V}\right)=0$. Isso significa que $X_{p}^{V}=0$, de modo que $X_{p}$ é um vetor horizontal, ou seja, $X_{p} \in H_{p}$. Dessa forma, a correspondência entre conexões e 1-forma de conexões é uma bijeção.

\subsubsection{Trasporte paralelo em fibrados principais}

Definição 2.4.4. Seja $\pi: P \rightarrow M$ um G-fibrado principal e $\gamma: I \rightarrow M$ uma curva. Um levantamento de $\gamma$ é uma curva $\tilde{\gamma}: I \rightarrow P$ tal que $\pi(\tilde{\gamma}(t))=$ $\gamma(t)$

Definição 2.4.5. Se $\pi: P \rightarrow M$ é um G-fibrado principal e $H \subseteq T P$ uma conexão, uma curva $\tilde{\gamma}: I \rightarrow P$ é horizontal se $\tilde{\gamma}^{\prime}(t) \in H_{\gamma(t)}$.

Definição 2.4.6. Um levantamento horizontal de $\gamma: I \rightarrow P$ é um levantamento $\tilde{\gamma}: I \rightarrow P$ que é uma curva horizontal em $P$.

Lema 2.4.2. Seja $X:\left[t_{0}, t_{1}\right] \rightarrow \mathfrak{g}$ uma curva. Então existe uma única curva suave $g:\left[t_{0}, t_{1}\right] \rightarrow G$ tal que $g^{\prime}(t) g(t)^{-1}=X(t), \forall t \in\left[t_{0}, t_{1}\right]$.

Demonstração. Ver [KN63], páginas 69 e 70.

Lema 2.4.3. Seja $\gamma:\left[t_{0}, t_{1}\right] \rightarrow M$ uma curva sobre $M$. Então para $p_{0} \in$ $P_{\gamma\left(t_{0}\right)}, t_{0} \in I$, existe um único levantamento horizontal $\tilde{\gamma}: I \rightarrow P$ de $\gamma$ em $P$ tal que $\tilde{\gamma}\left(t_{0}\right)=p_{0}$

Demonstração. (Retirado de [KN63]) 
Seja $\beta:\left[t_{0}, t_{1}\right] \rightarrow M$ uma curva tal tal que $\pi(\beta)=\gamma$ e $\beta\left(t_{0}\right)=p_{0}$. Então $\tilde{\gamma}(t)=\beta(t) g(t)$ é uma levantamento de $\gamma$,com $g:\left[t_{0}, t_{1}\right] \rightarrow G$ uma curva em $G$ tal que $g\left(t_{0}\right)=e$. Então

$$
\tilde{\gamma}^{\prime}(t)=\beta^{\prime}(t) g(t)+\beta(t) g^{\prime}(t)
$$

Seja $\omega$ uma 1-forma de conexão em $P$. Assim

$$
\begin{aligned}
\omega\left(\tilde{\gamma}^{\prime}(t)\right) & =\omega\left(\beta^{\prime}(t) g(t)\right)+\omega\left(\beta(t) g^{\prime}(t)\right) \\
& =\omega\left(R_{g(t)}\left(\beta^{\prime}(t)\right)+\omega\left(\beta(t) g^{\prime}(t)\right)\right. \\
& =A d_{g(t)^{-1}} \omega\left(\beta^{\prime}(t)\right)+g(t)^{-1} g^{\prime}(t)
\end{aligned}
$$

A curva $\tilde{\gamma}$ é horizontal se $\omega\left(\tilde{\gamma}^{\prime}(t)\right)=0$, isto é

$$
A d_{g(t)^{-1}} \omega\left(\beta^{\prime}(t)\right)+g(t)^{-1} g^{\prime}(t)=0
$$

Dessa forma

$$
\begin{aligned}
g(t)^{-1} g^{\prime}(t) & =-A d_{g(t)^{-1}} \omega\left(\beta^{\prime}(t)\right) \Rightarrow \\
A d_{g(t)}\left(g(t)^{-1} g^{\prime}(t)\right) & =A d_{g(t)}\left(-A d_{g(t)^{-1}} \omega\left(\beta^{\prime}(t)\right) \Rightarrow\right. \\
g^{\prime}(t) g(t)^{-1} & =-\omega\left(\beta^{\prime}(t)\right)
\end{aligned}
$$

E a solução existe de acordo com o lema anterior.

Definição 2.4.7. O transporte paralelo ao longo de $\gamma: I \rightarrow M$, do tempo $t_{0} \in I$ ao tempo $t_{1} \in I$ é a função

$$
\begin{gathered}
\mathcal{P}_{\gamma}^{t_{0}, t_{1}}: P_{\gamma\left(t_{1}\right)} \rightarrow P_{\gamma\left(t_{0}\right)} \\
\mathcal{P}_{\gamma}^{t_{0}, t_{1}}\left(p_{0}\right)=\tilde{\gamma}\left(t_{1}\right)
\end{gathered}
$$

com $\tilde{\gamma}$ o único levantamento horizontal de $\gamma$ tal que $\tilde{\gamma}\left(t_{0}\right)=p_{0}$.

\subsubsection{Curvatura.}

Consideremos o colchete de Lie [,] da álgebra e Lie $\mathfrak{g}$. Ele se estende a um colchete

$$
[., .]: \Omega^{1}(P, \mathfrak{g}) \times \Omega^{1}(P, \mathfrak{g}) \rightarrow \Omega^{2}(P, \mathfrak{g})
$$




$$
[u, v](X, Y)=[u(X), v(Y))]-[u(Y), v(X)]
$$

Sejam $X, Y \in T P$ que podemos estender a $\tilde{X}, \tilde{Y} \in \mathfrak{X}(P)$. Podemos considerar a parte horizontal destes campos vetoriais $\tilde{X}^{H}, \tilde{Y}^{H} \in \Gamma(H), H$ uma conexão.

Definição 2.4.8. Se $\omega$ é uma 1-forma de conexão em $P$ então a forma curvatura é $R \omega \in \Omega^{2}(P, \mathfrak{g})$ tal que

$$
R_{\omega}(X, Y)=d \omega\left(X^{H}, Y^{H}\right)
$$

Proposição 2.4.6. $R_{\omega}=0 \Longleftrightarrow\left[\tilde{X}^{H}, \tilde{Y}^{H}\right]$ é horizontal (ou seja, a forma curvatura mede a falha na integrabilidade de distribuições horizontais.)

Demonstração. Temos que $d \omega(X, Y)=X \omega(Y)-Y \omega(X)-\omega[X, Y]$. Então

$$
d \omega\left(X^{H}, Y^{H}\right)=\tilde{X}^{H} \omega\left(\tilde{Y}^{H}\right)-\tilde{Y}^{H} \omega\left(\tilde{X}^{H}\right)-\omega\left[\tilde{X}^{H}, \tilde{Y}^{H}\right]=-\omega\left[\tilde{X}^{H}, \tilde{Y}^{H}\right]
$$

Se $\left[\tilde{X}^{H}, \tilde{Y}^{H}\right]$ é horizontal, então $\omega\left[\tilde{X}^{H}, \tilde{Y}^{H}\right]=0$, de modo que $R_{\omega}=0$.

Se $R_{\omega}=0$, então $\omega\left[\tilde{X}^{H}, \tilde{Y}^{H}\right]=0$, o que significa que $\left[\tilde{X}^{H}, \tilde{Y}^{H}\right]$ é horizontal.

Proposição 2.4.7.

$$
R_{\omega}(X, Y)=d \omega(X, Y)+\frac{1}{2}[\omega, \omega](X, Y)
$$

Demonstração. Primeiramente, observemos que

$$
\begin{aligned}
{[\omega, \omega](X, Y) } & =[\omega(X), \omega(Y)]-[\omega(Y), \omega(X)] \\
& =[\omega(X), \omega(Y)]+[\omega(X), \omega(Y)] \\
& =2[\omega(X), \omega(Y)]
\end{aligned}
$$

Desse modo,

$$
d \omega(X, Y)+\frac{1}{2}[\omega, \omega](X, Y)=d \omega(X, Y)+[\omega(X), \omega(Y)]
$$

Então, devemos provar que

$$
d \omega\left(X^{H}, Y^{H}\right)=d \omega(X, Y)+[\omega(X), \omega(Y)]
$$


Agora, vamos considerar três casos:

- $X$ e $Y$ horizontais $\left(X=X^{H}, Y=Y^{H}\right)$ : então $\omega(X)=\omega(Y)$ de modo que

$$
d \omega(X, Y)+\frac{1}{2}[\omega, \omega](X, Y)=d \omega\left(X^{H}, Y^{H}\right)=R \omega(X, Y)
$$

- $X$ e $Y$ são verticais: então existem $\alpha, \beta \in \mathfrak{g}$ tais que $\psi(\alpha)=X$ e $\psi(\beta)=Y$. Então

$$
\begin{aligned}
d \omega(X, Y)+[\omega(X), \omega(Y)] & =X \omega(Y)-Y \omega(X)-\omega[X, Y]+[\omega(X), \omega(Y)] \\
& =X \omega(\psi(\beta))-Y \omega(\psi(\alpha))-\omega[\psi(\alpha), \psi(\beta)]-[\omega(\psi(\alpha)), \omega(\psi(\beta))] \\
& =-\omega(\psi([\alpha, \beta]))+[\alpha, \beta] \\
& =[\alpha, \beta]+[\alpha, \beta] \\
& =0
\end{aligned}
$$

Além disso, $d \omega\left(X^{H}, Y^{H}\right)=0$ pois $X$ e $Y$ são verticais, de modo que

- $X$ vertical e $Y$ horizontal: novamente, podemos assumir que $X=$ $\psi(\alpha), \alpha \in \mathfrak{g} . \operatorname{Assim} d \omega\left(X^{H}, Y^{H}\right)=0$ pois $X$ é vertical.

Além disso,

$$
\begin{aligned}
d \omega(X, Y)+[\omega(X), \omega(Y)] & =d \omega(\psi(\alpha), Y)+\omega[\psi(\alpha), Y]+[\omega(X), \omega(Y)] \\
& =\psi(\alpha) \omega(Y)-Y \omega(\psi(\alpha))-\omega[\psi(\alpha), Y]+[\omega(\psi(\alpha)), \omega(Y)] \\
& =-\omega[\psi(\alpha), Y]
\end{aligned}
$$

Agora, precisamos provar que se $V_{p}$ é vertical e $H_{p}$ é horizontal, então $\omega[\tilde{V}, \tilde{H}]=0$ Pelas fórmulas de Cartan

$$
\begin{aligned}
\omega[\tilde{V}, \tilde{H}] & =i_{[\tilde{V}, \tilde{H}]} \omega \\
& =\left[\mathcal{L}_{\tilde{V}}, i_{\tilde{V}}\right] \omega \\
& =i_{\tilde{H}} \mathcal{L}_{\tilde{V}} \omega \\
& =0
\end{aligned}
$$




\subsection{Conexões em fibrados vetoriais.}

Definição 2.5.1. Seja $\pi: E \rightarrow M$ um fibrado vetorial. Uma conexão em é uma função $\nabla$

$$
\begin{gathered}
\nabla: \mathfrak{X}(M) \times \Gamma(E) \rightarrow \Gamma(E) \\
\nabla(X, s)=\nabla_{X}(s)
\end{gathered}
$$

com as seguintes propriedades:

1. $\nabla$ é $\mathbb{R}$-bilinear;

2. $\nabla_{f X}(s)=f \nabla_{X}(s)$;

3. $\nabla_{X}(g s)=g \nabla_{X}(s)+X(g) s$

$\operatorname{com} f, g \in C^{\infty}(M), X \in \mathfrak{X}(M), s \in \Gamma(E)$

Lema 2.5.1. O fibrado vetorial trivial admite uma conexão.

Demonstração. Se $E=M \times \mathbb{R}^{k}$ então $\Gamma(E)=C^{\infty}\left(M, \mathbb{R}^{k}\right)$. Assim, podemos definir a seguinte conexão

$$
\nabla_{X}(s)=d s(X)
$$

As propriedades de conexão decorrem todas das propriedades da diferencial $d s$.

Lema 2.5.2. Se $\nabla^{1} e \nabla^{2}$ são ambas conexões sobre fibrado vetorial $\pi: \rightarrow P$ e $\lambda_{1}, \lambda_{2}$ são funções suaves em $M$ tais que $\lambda_{1}+\lambda_{2}=1$. Então $\lambda_{1} \nabla^{1}+\lambda_{2} \nabla^{2}$ é uma conexão sobre os mesmo fibrado vetorial.

Demonstração. A $\mathbb{R}$-bilinearidade de $\nabla:=\lambda_{1} \nabla^{1}+\lambda_{2} \nabla^{2}$ advém do fato de ela ser combinação de duas funções bilineares.

Seja $f \in C^{\infty}(M)$ :

$$
\begin{aligned}
\nabla_{f} X(s) & =\lambda_{1} \nabla_{f}^{1} X(s)+\lambda_{2} \nabla_{f}^{2} X(s) \\
& =\lambda_{1} f \nabla_{X}^{1}(s)+\lambda_{2} f \nabla_{f}^{2} X(s) \\
& =f\left(\lambda_{1} \nabla_{X}^{1}(s)+\lambda_{2} \nabla_{X}^{2}\right) \\
& =f \nabla_{X}(s)
\end{aligned}
$$


Seja $g \in C^{\infty}(M)$. Então:

$$
\begin{aligned}
\nabla_{X}(g s) & =\lambda_{1} \nabla_{X}^{1}(g s)+\lambda_{2} \nabla_{X}^{2}(g s) \\
& =\lambda_{1}\left[X(g) s+g \nabla_{X}^{1}(s)\right]+\lambda_{2}\left[X(g) s+g \nabla_{X}^{2}(s)\right] \\
& =\left(\lambda_{1}+\lambda_{2}\right) X(g) s+g\left[\lambda_{1} \nabla_{X}^{1}(s)+\lambda_{2} \nabla_{X}^{2}(s)\right] \\
& =X(g) s+g\left(\lambda_{1} \nabla_{+}^{1} \lambda_{2} \nabla^{2}\right)_{X}(s) \\
& =X(g) s+g \nabla_{X}(s)
\end{aligned}
$$

Proposição 2.5.1. Todo fibrado vetorial admite conexão.

Demonstração. Consideremos uma cobertura aberta $\left\{U_{\lambda}\right\}$ de $M$ de modo que $E_{U_{\lambda}}$ é trivializável e consideremos $\left\{\rho_{\lambda}\right\}$ uma partição da unidade subordinada a cobertura anterior. Sejam $\nabla$ a conexão sobre $E_{\lambda}$. Então

$$
\nabla_{X}(s):=\left.\sum_{\lambda} \rho_{\lambda} \nabla \lambda_{X}\right|_{U_{\lambda}}\left(\left.s\right|_{U_{\lambda}}\right)
$$

Proposição 2.5.2. Seja $\nabla$ uma conexão sobre um fibrado vetorial $\pi: E \rightarrow$ $P$ e $s, s^{\prime} \in \Gamma(E)$ seções tais que $\left.s\right|_{U},\left.s^{\prime}\right|_{U}$, e $X$ e $X^{\prime}$ campos vetoriais tais que $X\left|U=X^{\prime}\right| U^{\prime}$, em $U$ vizinhança aberta de $p \in M$. Então:

$$
\left.\nabla_{X}(s)\right|_{U}=\left.\nabla_{X^{\prime}}\left(s^{\prime}\right)\right|_{U}
$$

Demonstração. . A bilinearidade da conexão reduz a prova à duas situações:

1. se $\left.s\right|_{U}=0$ então $\nabla_{X}(s)=0$ para toda $X \in \mathfrak{X}(M)$.

2. se $\left.X\right|_{U}=0$ então $\nabla_{X}(s)=0$ para toda $s \in \Gamma(E)$.

Seja $\eta: U \rightarrow[0,1]$ uma "bump function". Então:

1. $\left.s\right|_{U}=\left.0 \Rightarrow \eta s\right|_{U}=0$. Então, para $x_{0} \in U$,

$$
\begin{aligned}
\nabla_{X} \eta s\left(x_{0}\right) & =0 \Rightarrow \\
\left(X(\eta)+\eta \nabla_{X} s\right)\left(x_{0}\right) & =0 \Rightarrow \\
\eta\left(x_{0}\right) \nabla_{X} s\left(x_{0}\right) & =0 \Rightarrow \\
\nabla_{X} s\left(x_{0}\right) & =0
\end{aligned}
$$


2. $\left.s\right|_{U}=\left.0 \Rightarrow \eta s\right|_{U}=0$. Então, para $x_{0} \in U$,

$$
\begin{aligned}
\eta X & =0 \Rightarrow \\
\nabla_{X} \eta s\left(x_{0}\right) & =0 \Rightarrow \\
\left(\eta \nabla_{X} s\right)\left(x_{0}\right) & =0 \Rightarrow \\
\eta\left(x_{0}\right)\left(\nabla_{X} s\right)\left(x_{0}\right) & =0 \Rightarrow \\
\left(\nabla_{X} s\right)\left(x_{0}\right) & =0
\end{aligned}
$$

Definição 2.5.2. Seja $f^{*} E$ um fibrado pullback. Definimos a conexão pullback como

$$
\left(f^{*} \nabla\right)_{Y}\left(f^{*} s\right)=f^{*}\left(\nabla_{d f(Y)} s\right)
$$

para $Y \in \mathfrak{X}(N)$ e $s \in \Gamma(E)$.

Observação 7. Seja $\alpha \in \Gamma\left(f^{*} E\right)$ e $Y \in \mathfrak{X}(N)$. Então $\alpha=\sum u_{i}\left(f^{*} s_{i}\right)$, $s_{i} \in \Gamma(E) e$

$$
\begin{aligned}
\left(f^{*} \nabla\right)_{Y} \alpha & =\left(f^{*} \nabla\right)_{Y} \sum u_{i}\left(f^{*} s_{i}\right) \\
& =\sum\left(Y\left(u_{i}\right)\left(f^{*} s_{i}\right)+u_{i}\left(f^{*} \nabla\right)_{Y}\left(f^{*} s_{i}\right)\right) \\
& =\sum\left(Y\left(u_{i}\right)\left(f^{*} s_{i}\right)+u_{i}\left(f^{*}(\nabla)_{Y} s_{i}\right)\right.
\end{aligned}
$$

Observação 8. Dado um fibrado vetorial $E$ de posto $k$ e $\nabla$ uma conexão em $E$, então se $\left\{\varepsilon_{1}, \ldots, \varepsilon_{k}\right\}$ é um referencial local para um aberto $U \subseteq M$ podemos escrever

$$
\nabla_{X}\left(\varepsilon_{j}\right)=\sum_{i=1}^{k} \omega_{i j} \varepsilon_{j}
$$

de modo que a conexão fica determinada pela matriz

$$
\mu(\nabla, \varepsilon)=\left[\mu_{i j}\right]
$$

onde $\mu_{i j} \in \Omega^{1}(U)$. Esta matriz é chamada de matriz da conexão 


\subsubsection{Transporte paralelo em fibrados vetoriais.}

Seja $\gamma: I \rightarrow M$ uma curva. Se fizermos a construção do fibrado pullback de $E$ por $\gamma$, obtemos $\gamma^{*}(E)=\{(t, p) \in I \times E: \gamma(t)=\pi(p)\}$. Uma seção $\alpha \in \Gamma\left(\gamma^{*}(E)\right)$ é uma curva $\alpha: I \rightarrow \gamma^{*}(E) \operatorname{com} \alpha(t) \in \gamma^{*}(E)_{t}=E_{\gamma(t)}$, ou seja, $\pi(\alpha(t))=\gamma(t)$.

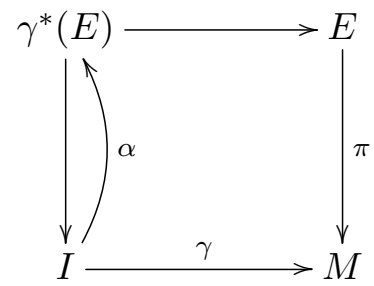

Definição 2.5.3. Uma secção $\alpha \in \Gamma\left(\gamma^{*}(E)\right)$ é chamada de seção ao longo de $\gamma$.

Exemplo 2.5.1. Se $\sigma$ é uma seção de um fibrado vetorial $\pi: E \rightarrow M e$ $\gamma: I \rightarrow M$ é uma curva em $M$, então $\sigma \circ \gamma: I \rightarrow M$ é uma seção de $E$ ao longo de $\gamma$.

Definição 2.5.4. Seja $\alpha \in \Gamma\left(\gamma^{*} E\right)$. A derivada covariante com respeito a conexão $\nabla$ ao longo de $\gamma$ é definida como

$$
\frac{\nabla \alpha}{d t}=\left(\gamma^{*} \nabla\right)_{\frac{d}{d t}} \alpha
$$

Definição 2.5.5. Uma seção $\alpha$ sobre $\gamma$ é paralela com respeito a $\nabla$ se $\frac{\nabla \alpha}{d t}=0, \forall t \in I$.

Lema 2.5.3. Sejm $\pi: E \rightarrow M$ é um fibrado vetorial $e \nabla$ uma conexão sobre ele com $\gamma$ uma curva suave em $M$, definida em I. Então existe uma única seção $\alpha$ sobre $\gamma$ que é paralelo e $\alpha\left(t_{0}\right)=\alpha_{0} \in E_{\gamma\left(t_{0}\right)}, t_{0} \in I$.

Demonstração. Seja $U$ um aberto em $M$ de modo que $E_{U}$ é trivializável. Seja $\left\{s_{1}, \ldots, s_{k}\right\}$ um referencial de $\left.E\right|_{U}$. Então existem funções $f_{i} \in C^{\infty}(I)$ de modo que $\alpha \in \Gamma\left(\gamma^{*}(E)\right)$ expressa-se com

$$
\alpha(t)=\sum_{i=1}^{k}=f_{i}(t) s_{i}(\gamma(t))=\sum_{i=1}^{k}=f_{i}(t)\left(\gamma^{*} s_{i}\right)(t)
$$


Então:

$$
\begin{aligned}
\frac{\nabla \alpha}{d t} & =\left(\gamma^{*} \nabla\right)_{\frac{d}{d t}}\left(\sum_{i=1}^{k} f_{i}(t) s_{i}(\gamma(t))\right) \\
& =\sum_{i=1}^{k} \frac{d f_{i}(t)}{d t}\left(\gamma^{*} s_{i}\right)(t)+\sum_{i=1}^{k} f_{i}(t)\left(\gamma^{*} \nabla\right)_{\frac{d}{d t}}\left(\gamma^{*} s_{i}\right)(t) \\
& =\sum_{i=1}^{k} f_{i}^{\prime}(t) s_{i}(\gamma(t))+\sum_{i=1}^{k} f_{i}(t) \sum_{j=1}^{k} g_{j}^{i} s_{j}(\gamma(t)) \\
& =\sum_{i=1}^{k}\left(f_{i}^{\prime}(t)+\sum_{j=1}^{k} f_{j}(t) g_{i}^{j}\right) s_{i}(\gamma(t))
\end{aligned}
$$

$\operatorname{com}\left(\gamma^{*} \nabla\right)_{\frac{d}{d t}}\left(\gamma^{*} s_{i}\right)(t)=\sum_{j=1}^{k} g_{j}^{i} s_{j}(\gamma(t)), g_{j}^{i} \in C^{\infty}(I)$ já que $\left(\gamma^{*} \nabla\right)_{\frac{d}{d t}}\left(\gamma^{*} s_{i}\right) \in$ $\Gamma\left(\gamma^{*}(E)\right)$.

Se $\alpha$ é paralelo, então $\frac{\nabla \alpha}{d t}=0$ e, consequentemente

$$
f_{i}^{\prime}(t)+\sum_{j=1}^{k} f_{j}(t) g_{i}^{j}=0 \Rightarrow f_{i}^{\prime}(t)=-\sum_{j=1}^{k} f_{j}(t) g_{i}^{j}
$$

para cada $i=1, \ldots, k$ com $\alpha\left(t_{0}\right)=\alpha_{0}$

Este é um sistema de equações diferencias ordinárias de primeira ordem, cuja solução única é garantida pelo Teorema de Existência e Unicidade.

Definição 2.5.6. Seja $\pi: E \rightarrow M$ um fibrado vetorial $e \gamma: I \rightarrow M$ uma curva em M.O transporte paralelo ao longo de $\gamma: I \rightarrow M$, do tempo $t_{0} \in I$ ao tempo $t_{1} \in I$ com respeito a conexão $\nabla$ é a função

$$
\begin{gathered}
\mathcal{P}_{\gamma}^{t_{0}, t_{1}}: \pi^{-1}\left(\gamma\left(t_{0}\right)\right) \rightarrow \pi^{-1}\left(\gamma\left(t_{0}\right)\right) \\
\mathcal{P}_{\gamma}^{t_{0}, t_{1}}\left(\alpha_{0}\right)=\alpha_{1}
\end{gathered}
$$

com $\alpha$ a única seção ao longo de $\gamma$ paralela sobre $\gamma$ tal que $\alpha\left(t_{0}\right)=\alpha_{0}$. 


\subsection{Curvatura e torção.}

\subsubsection{Curvatura.}

Definição 2.6.1. Se $\nabla$ é uma conexão sobre um fibrado vetorial, a função

$$
\begin{gathered}
R: \mathfrak{X}(M) \times \mathfrak{X}(M) \times \Gamma(E) \rightarrow \Gamma(E) \\
R(X, Y)(s)=\nabla_{X} \nabla_{Y}(s)-\nabla_{Y} \nabla_{X}(s)-\nabla_{[X, Y]}(s)
\end{gathered}
$$

é chamada de curvatura de $\nabla$.

Proposição 2.6.1. $R \in \Gamma\left(\Lambda^{2} T^{*} M \otimes E^{*} \otimes E\right)$

Demonstração. Provar que $R \in \Gamma\left(\Lambda^{2} T^{*} M \otimes E^{*} \otimes E\right)$ significa provar que $R$ é tensorial e antissimétrica.

1. A linearidade de cada parcela é consequência da linearidade da conexão e do colchete.

2. Sendo $f \in C^{\infty}(M)$,

$$
\begin{aligned}
R(f X, Y)(s) & =\nabla_{f X} \nabla_{Y}(s)-\nabla_{Y} \nabla_{f X}(s)-\nabla_{[f X, Y]}(s) \\
& =f \nabla_{X} \nabla_{Y}(s)-\nabla_{Y} f \nabla_{X}(s)+\nabla_{[Y, f X]}(s) \\
& =f \nabla_{X} \nabla_{Y}(s)-Y(f) \nabla_{X}(s)-f \nabla_{Y} \nabla_{X}+\nabla_{Y(f) X+f[X, Y]}(s) \\
& =f \nabla_{X} \nabla_{Y}(s)-Y(f) \nabla_{X}(s)-f \nabla_{Y} \nabla_{X}+Y(f) \nabla_{X}(s)+f \nabla_{[X, Y]}(s) \\
& =f\left(\nabla_{X} \nabla_{Y}(s)-\nabla_{Y} \nabla_{X}+\nabla_{[X, Y]}(s)\right) \\
& =f R(X, Y)(s)
\end{aligned}
$$

Analogamente prova-se que $R(X, f Y)=f R(X, Y)$.

3. Sendo $g \in C^{\infty}(M)$,

$$
\begin{aligned}
R(X, Y)(g s) & =\nabla_{X} \nabla_{Y}(g s)-\nabla_{Y} \nabla_{X}(g s)-\nabla_{[X, Y]}(g s) \\
& =\nabla_{X}\left(Y(g) s+g \nabla_{Y}(s)\right)-\nabla_{Y}\left(X(g) s+g \nabla_{X}(s)\right)-[X, Y](g) s-g \nabla_{[X, Y]}(s) \\
& =X Y(g) s+Y(g) \nabla_{X}(s)+X(g) \nabla_{Y}(s)+g \nabla_{X} \nabla_{Y}(s) \\
& -Y X(g) s-X(g) \nabla_{Y}(s)-Y(g) \nabla_{X}(s)-g \nabla_{Y} \nabla_{X}(s) \\
& -X Y(g) s+Y X(g) s-g \nabla_{[X, Y]}(s) \\
& =g \nabla_{X} \nabla_{Y}(s)-g \nabla_{Y} \nabla_{X}(s)-g \nabla_{[X, Y]}(s) \\
& =g R(X, Y)(s)
\end{aligned}
$$


4. Vamos provar a antissimetria:

$$
\begin{aligned}
R(X, Y)(s) & =\nabla_{X} \nabla_{Y}(s)-\nabla_{Y} \nabla_{X}(s)-\nabla_{[X, Y]}(s) \\
& =\nabla_{X} \nabla_{Y}(s)-\nabla_{Y} \nabla_{X}(s)+\nabla_{[Y, X]}(s) \\
& =-\left(\nabla_{Y} \nabla_{X}(s)-\nabla_{X} \nabla_{Y}(s)+\nabla_{[Y, X]}(s)\right) \\
& =-R(Y, X)(s)
\end{aligned}
$$

\subsubsection{Torção}

Ao trabalharmos com fibrados tangentes $\pi: T M \rightarrow M$, que são fibrados vetoriais, temos que $\mathfrak{X}(M)=\Gamma(T M)$, de modo que uma conexão será uma função $\nabla: \mathfrak{X}(M) \times \mathfrak{X}(M) \rightarrow \mathfrak{X}(M)$.

Definição 2.6.2. Dada uma conexão $\nabla$ sobre um fibrado vetorial, a função

$$
\begin{gathered}
T: \mathfrak{X}(M) \times \mathfrak{X}(M) \rightarrow \mathfrak{X}(M) \\
T(X, Y)=\nabla_{X} Y-\nabla_{Y} X-[X, Y]
\end{gathered}
$$

é a torção de uma conexão $\nabla$.

Proposição 2.6.2. $T \in \Gamma\left(\Lambda^{2} T^{*} M \otimes T M\right)$

Demonstração. A linearidade sobre $\mathbb{R}$ em cada coordenada advém da linearidade da conexão e do colchete. Vamos provar que $T$ é tensorial e antissimétrica:

$$
\begin{aligned}
T(f X, Y) & =\nabla_{f X} Y-\nabla_{Y} f X-[f X, Y] \\
& =f \nabla_{X} Y-Y(f) X-f \nabla_{Y} X+[Y, f X] \\
& =f \nabla_{X} Y-Y(f) X-f \nabla_{Y} X+Y(f) X+f[Y, X] \\
& =f \nabla_{X} Y-f \nabla_{Y} X-f[X, Y] \\
& =f T(X, Y)
\end{aligned}
$$

Analogamente prova-se que $T(X, f Y)=f T(X, Y)$. 


$$
\begin{aligned}
T(X, Y) & =\nabla_{X} Y-\nabla_{Y} X-[X, Y] \\
& =-\nabla_{Y} X+\nabla_{X} Y+[Y, X] \\
& =-\left(\nabla_{Y} X-\nabla_{X} Y-[Y, X]\right) \\
& =-T(Y, X)
\end{aligned}
$$

\subsubsection{Quando a curvatura é zero.}

Seja $\pi: E \rightarrow M$ um fibrado vetorial. Seja

$$
\Omega^{k}(M, E)=\Gamma\left(\Lambda^{k} T^{*} M \otimes E\right)
$$

e $\omega \in \Omega^{p}(M), \tau \in \Omega^{q}(M, E)$. Então

$$
(\omega \wedge \tau)\left(X_{1}, \cdots, X_{p+q}\right)=\sum_{\sigma} \operatorname{sgn}(\sigma) \omega\left(X_{\sigma(1)}, \ldots, X_{\sigma(p)}\right) \tau\left(X_{\sigma(p+1), \ldots, X_{\sigma(p+q)}}\right)
$$

com $\sigma(1)<\ldots<\sigma(p)$ e $\sigma(p+1)<\ldots<\sigma(p+q)$. Ou seja, $\Omega^{\bullet}(M, E)$ é um módulo sobre $\Omega^{\bullet}(M)$.

Definição 2.6.3. A derivada exterior é definida como o operador

$$
d_{\nabla}: \Omega^{\bullet}(M, E) \rightarrow \Omega^{\bullet+1}(M, E)
$$

tal que

$$
\begin{aligned}
d_{\nabla}(\tau)\left(X_{0}, \ldots, X_{q}\right) & =\sum_{0}^{q}(-1)^{i} \nabla_{X_{i}} \tau\left(X_{0}, \ldots, \hat{X}_{i}, \ldots, X_{q}\right)+ \\
& +\sum_{0 \leq i<j \leq q}(-1)^{i+j} \tau\left(\left[X_{i}, X_{j},\right] X_{0}, \ldots, \hat{X}_{i}, \ldots, \hat{X}_{j}, \ldots, X_{q}\right)
\end{aligned}
$$

Além disso,

$$
d_{\nabla}(\omega \wedge \tau)=(d \omega) \wedge \tau+(-1)^{|\omega|} \omega \wedge d_{\nabla} \tau
$$


em que $|\omega|$ é o grau de $\omega$. Agora, se é dado $d: \Omega^{\bullet}(M, E) \rightarrow \Omega^{\bullet+1}(M, E)$, como obtemos uma conexão $\nabla$ a ele associado?

Temos que $\Omega^{0}(M, E)=\Gamma(E)$. Desse modo, se $s \in \Gamma(E)$, então $d s \in$ $\Omega^{1}(M, E)$. Definindo-se

$$
\nabla_{X}(s)=d s(X)
$$

temos:

- $\nabla_{f X}(s)=f d s(X)=f \nabla_{X}(s)$

- $\nabla_{X}(g s)=d(g s)(X)=(d g \wedge s)(X)+(-1)^{0} g \wedge d s(X)=d f(X) s+f \nabla_{X}(s)$

para $f, g$ funções suaves em $M$. Assim, tal $\nabla$ é uma conexão.

Exemplo 2.6.1. Se $\nabla$ é uma conexão em $E=T M$, consideremos a 1-forma identidade $\mathbb{1} \in \Omega^{1}(M, T M)$.

Então

$$
d_{\nabla}(\mathbb{1}) \in \Omega^{2}(M, T M)=\Gamma\left(\Lambda^{2} T^{*} M \otimes T M\right)
$$

$e$

$$
\begin{aligned}
d_{\nabla}(\mathbb{1})\left(X_{0}, X_{1}\right) & =(-1)^{0} \nabla_{X_{0}} X_{1}+(-1)^{1} \nabla_{X_{1}} X_{0}+(-1)^{0+1}\left[X_{0}, X_{1}\right] \\
& =\nabla_{X_{0}} X_{1}-\nabla_{X_{1}} X_{0}-\left[X_{0}, X_{1}\right] \\
& =T\left(X_{0}, X_{1}\right)
\end{aligned}
$$

esta, a torção da conexão $\nabla$.

Vamos considerar agora o operador $d_{\nabla}^{2}: \Omega^{\bullet}(M, E) \rightarrow \Omega^{\bullet+2}(M, E)$

Proposição 2.6.3. Seja $\nabla$ uma conexão e $\omega$ uma $k$-forma. Então $d_{\nabla}^{2}(\omega)=$ $R_{\nabla} \wedge \omega$

Demonstração. Se $\omega \in \Omega^{p}(M)$ e $\tau \in \Omega^{k}(M, E)$, então

$$
\begin{aligned}
d_{\nabla}^{2}(\omega \wedge \tau) & =d_{\nabla}\left((d \omega) \wedge \tau+(-1)^{p} \omega \wedge d_{\nabla} \tau\right) \\
& =d_{\nabla}((d \omega) \wedge \tau)(-1)^{p} d_{\nabla}\left(\omega \wedge d_{\nabla} \tau\right) \\
& =\left(d^{2}(\omega) \wedge \tau+(-1)^{p+1} d(\omega) \wedge d_{\nabla}(\tau)\right)+(-1)^{p}\left[d(\omega) \wedge d_{\nabla}(\tau)+(-1)^{p} \omega \wedge d_{\nabla}^{2}(\tau)\right] \\
& =\underbrace{d^{2}(\omega) \wedge \tau+(-1)^{2 p} \omega \wedge d_{\nabla}^{2}(\tau)}_{=0} \\
& =\omega \wedge d_{\nabla}^{2}(\tau)
\end{aligned}
$$


Seja $s \in \Gamma(E)$ uma 0-forma. Então:

$$
\begin{aligned}
d_{\nabla}^{2} s(X, Y) & =d_{\nabla}\left(d_{\nabla} s\right)(X, Y) \\
& =\nabla_{X}\left(d_{\nabla} s(X)\right)-\nabla_{Y}\left(d_{\nabla} s(Y)\right)-\nabla_{[X, Y]} s \\
& =R_{\nabla} s(X, Y)
\end{aligned}
$$

Seja $\omega \in \Omega(M, E)$ e $\left\{s_{1}, \ldots, s_{k}\right\}$ um referencial local de $E$ então cujo domínio é $U$, então:

$$
\left.\omega\right|_{U}=\sum \omega_{i}^{U} s_{i}
$$

$\operatorname{com} \omega_{i}^{U} \in \Omega^{k}(U)$

Então,

$$
\begin{aligned}
\left.d_{\nabla}^{2} \omega\right|_{U} & =\sum \omega_{i}^{U} \wedge d_{\nabla}^{2} s_{i} \\
& =\sum \omega_{i}^{U} \wedge R_{\nabla} s_{i} \\
& =\sum(-1)^{2 k} R_{\nabla} \wedge \omega_{i}^{U} s_{i} \\
& =\left.R_{\nabla} \wedge \omega\right|_{U}
\end{aligned}
$$

Corolário 2.6.1. $d_{\nabla}^{2}=0 \Longleftrightarrow R_{\nabla}=0$

\subsubsection{Formas em fibrados associados}

Consideremos $E(P, V)$ um fibrado associado.

Seja $\omega \in \Omega^{1}(P, V)$ tal que

$$
\left\{\begin{array}{l}
i_{\xi}(\omega)=0, \text { se } \xi \text { é vertical } \\
R_{g}^{*} \omega_{p}=g^{-1} \omega_{p g}
\end{array}\right.
$$

Então $\omega$ induz $\bar{\omega} \in=\Omega(M, E(P, V))$.

Definamos $\bar{\omega}_{x}(X)=\left[p, \omega_{p}\left(h_{p} X\right)\right]$, com $x=\pi(p)$, onde $h_{p}$ é um levantamento horizontal. Vamos mostrar que está bem definida essa forma. 
Primeiro vamos fixar a conexão.Então:

$$
\begin{aligned}
\bar{\omega}_{x}(X) & =\left[p g, \omega_{p g}\left(h_{p g} X\right)\right] \\
& =\left[p, g \omega_{p g}\left(h_{p g} X\right)\right] \\
& \left.=\left[p, g \omega_{p g}\left(d R_{g}\right)_{p}\left(h_{p} X\right)\right)\right] \\
& =\left[p, g R_{g}^{*} \omega_{p}\left(h_{p} X\right)\right] \\
& =\left[p, g g^{-1} \omega_{p}\left(h_{p} X\right)\right] \\
& =\left[p, \omega_{p}\left(h_{p} X\right)\right]
\end{aligned}
$$

de modo que $\omega$ estava bem definida quanto ao representante na fibra.

Agora, mostremos que não há dependência da conexão: seja $\bar{\omega}^{\prime}{ }_{x}(X)=$ $\left[p, \omega_{p}\left(h_{p}^{\prime} X\right)\right] ;$ então:

$$
\left[\bar{\omega}_{x}-\bar{\omega}^{\prime}{ }_{x}\right](X)=[p, \omega \underbrace{\left(h_{p} X-h_{p}^{\prime} X\right)}_{\text {diferença de vetores horizontais é vertical }}]=[p, 0] \Rightarrow \bar{\omega}=\bar{\omega}^{\prime}
$$

\subsection{Conexões induzidas em fibrados associados}

Vamos considerar um $G$-fibrado principal $\pi: P \longrightarrow M$ e $\rho: G \longrightarrow$ $G L(V)$ uma representação de G seja $H$ é uma conexão em $P$.

Proposição 2.7.1. H induz uma conexão no fibrado associado $E(P, V)$.

Demonstração. (Baseado em [Bel]) Se diferenciarmos a ação em $e$, obtemos a aplicação $\bar{\rho}: \mathfrak{g} \rightarrow \operatorname{End}(V)$. Se tomarmos $X, Y \in \mathfrak{g}$ e [, $]_{\operatorname{End}(V)}$ o comutador em $\operatorname{End}(V)$, então

$$
[\bar{\rho}(X), \bar{\rho}(X)]_{\operatorname{End}(V)}=\bar{\rho}[X, Y]
$$

Se $s \in \Gamma(E(P, V))$ e $\sigma \in \Gamma(U, P), U$ aberto em $M$, então vamos definir $u: U \longrightarrow V$ tal que $s(x)=[\sigma(x), u(x)]$.

Definamos

$$
\nabla(s)=\left[\sigma, \bar{\rho}\left(\sigma^{*} \omega\right)(u)+d u\right]
$$

onde $\omega$ a 1-forma de conexão associada a $H$.

Vamos provar que a definição acima independe da escolha da seção em $U$. Se $\sigma_{1}, \sigma_{2} \in \Gamma(U, P)$, então existe $g: U \longrightarrow G$ tal que $\sigma_{1}(x)=\sigma_{2}(x) g(x)$, 
para $x \in U$. Além disso, correspondem, respectivamente a cada seção, $u_{1} \mathrm{e}$ $u_{2}$, de modo que $u_{2}=\rho\left(g^{-1}\right) u_{1}$

$$
\begin{aligned}
{\left[\sigma_{2}, \bar{\rho}\left(\sigma_{2}^{*} \omega\right)\left(u_{2}\right)+d u_{2}\right]=} & {\left[\sigma_{1} g, \bar{\rho}\left(\left(\sigma_{1} g\right)^{*} \omega\right)\left(u_{1}\right)+d\left(\rho\left(g^{-1}\right) u_{2}\right)\right] } \\
= & {\left[\sigma_{1} g, \bar{\rho}\left(A d_{\rho^{-1}}(\omega)\left(\rho\left(g^{-1}\right)\left(u_{1}\right)\right)+\bar{\rho}\left(g^{*} \omega_{M C}\left(u_{1}\right)\right)-\right.\right.} \\
& \left.-\bar{\rho}\left(g^{*} \omega_{M C}\left(u_{1}\right)\right)+\rho\left(g^{-1}\left(d u_{1}\right)\right)\right] \\
= & {\left[\sigma_{1}, \bar{\rho}\left(\sigma_{1}^{*} \omega\right)\left(u_{1}\right)+d u_{1}\right] }
\end{aligned}
$$

A regra de Leibniz é garantida pela presença de $d u$ na segunda coordenada da classe.

\subsection{Redução do grupo estrutural}

Seja $\pi: P \longrightarrow M$ um $G$-fibrado principal e $G^{\prime}$ um subgrupo de Lie $G$. Tal fibrado admite uma redução de seu grupo estrutural a $G^{\prime}$ se existe $P^{\prime} \subseteq P$ tal que $\left.\pi\right|_{P^{\prime \prime}}: P \longrightarrow M$ é um $H$-fibrado principal

Exemplo 2.8.1. $\quad$ 1. Se $G^{\prime}=\{e\}$ então uma redução será equivalente a uma trivialização .

2. Se $G^{\prime}=O(n)$, então uma redução é a escolha de um produto interno.

3. Se $G^{\prime}=G L_{n}^{+}(\mathbb{R})$, uma redução é a escolha de uma orientação. 
40 FIBRADOS PRINCIPAIS, VETORIAIS E ASSOCIADOS. 


\section{Capítulo 3}

\section{G-estruturas}

Nesse capítulo apresentaremos o que é uma G-estrutura e os principais exemplos.

Também discutiremos o conceito de carta adaptada à G-estrutura e de integrabilidade de G-estruturas. Esse é o conceito central que estamos estudando.

A curvatura e troção em G-estruturas são estruturas geométricas que podem nos ajudar na questão da integrabilidade.

No fim, com o auxílio da forma de Maurer-Cartan e a forma tautológica, vamos chegar uma uma condição necessária de integrabilidade.

Esse capítulo também teve base em [Cra15]

\subsection{G-estruturas em variedades}

Definição 3.1.1. Sejam $M$ uma variedade diferenciável de dimensão ne $G \subseteq G L_{n}(\mathbb{R})$ um subgrupo de Lie. Uma $\boldsymbol{G}$-estrutura em $M$ é subvariedade

$$
\mathcal{S} \subseteq \operatorname{Fr}(M)
$$

que é G-invariante e é um G-fibrado principal com relação à projeção $\pi$ : $\mathcal{S} \rightarrow M$ e à ação induzida de $G$.

Um elemnto de $\mathcal{S}$ é denominado G-referencial.

Definição 3.1.2. Dadas uma carta local $(U, \varphi)$ para $M\left(\frac{\partial}{\partial \varphi_{1}}, \ldots, \frac{\partial}{\partial \varphi_{n}}\right) \in$ $\Gamma_{U}(\operatorname{Fr}(M))$ é chamado de referencial induzido. 
Definição 3.1.3. Dada $(U, \varphi)$ uma carta local e $\mathcal{S}$ uma G-estrutura. Se

$$
\left(\frac{\partial}{\partial \varphi_{1}}(x), \ldots, \frac{\partial}{\partial \varphi_{n}}(x)\right) \in \mathcal{S}_{x}, x \in U
$$

o referencial é chamado de adaptado e respectiva carta local também e chamada adaptada. Uma G-estrutura é integrável se em torno de todo ponto $x \in M$ existe uma carta local adaptada.

\subsubsection{Problema da equivalência de G-estruturas}

Definição 3.1.4. Sejam duas G-estruturas $\mathcal{S}$ sobre $M$ e $\overline{\mathcal{S}}$ sobre $\bar{M}$ e $f: M \rightarrow \bar{M}$ um difeomorfismo. A função $f$ é isomorfismo entre $G$ estruturas se

$$
\overline{\mathcal{S}}_{f(x)}=f_{*} \mathcal{S}_{x}
$$

onde $f_{*}$ é a função induzida por $d_{x} f: T_{x} M \longrightarrow T_{f(x)} M$. No caso da existência de um isomorfismo, as $G$-estruturas $\mathcal{S}$ e $\overline{\mathcal{S}}$ são equivalentes.

Uma questão central da teoria de G-estruturas é o problema da equivalência local: quando existe um difeomorfismo local $f: U \rightarrow \bar{U}, U \subseteq M$ e $\bar{U} \subseteq M$ abertos de modo que $f_{*}$ seja um isomorfismo local entre Gestruturas.

A chamada $G$-estrutura padrão é aquela dada pelo referencial

$$
\left(\frac{\partial}{\partial x_{1}}, \ldots, \frac{\partial}{\partial x_{n}}\right)
$$

em $\mathbb{R}^{n}$ e será denotada pro $\mathcal{S}^{\text {stand }}$.

O problema da equivalência tem um caso particular que é a questão da integrabilidade. Esta procura as condições necessárias e suficientes da equivalência local entre $G$-estruturas e a $G$-estrutura padrão (também chamada de flat).

Assim, uma $G$-estrutura é integrável se, e somente se, é localmente equivalente $\mathcal{S}_{x}^{\text {stand }}$.

\subsubsection{Exemplos}

Exemplo 3.1.1 $(G=\{e\})$. O primeiro exemplo aborda o caso em que $G=\{e\}$. Uma G-estrutura, aqui chamada simplesmente e-estrutura, é um 
referencial global $\phi=\left(\phi_{1}, \ldots, \phi_{n}\right)$. Se $(U, \varphi)$ é uma carta local, a integrabilidade da e-estrutura implica que

$$
\phi_{i}=\frac{\partial}{\partial \varphi_{i}}, i=1, \ldots, n
$$

O modelo padrão flat é $\frac{\partial}{\partial x_{1}}, \ldots, \frac{\partial}{\partial x_{n}}$ em $\mathbb{R}^{n}$.

Se $\phi=\left(\phi_{1}, \ldots, \phi_{n}\right)$ é uma e-estrutura em $M$ e $\bar{\phi}=\left(\bar{\phi}_{1}, \ldots, \bar{\phi}_{n}\right)$ é uma e-estrutura em $\bar{M}$, um difeomorfismo $f: M \rightarrow \bar{M}$ será isomorfismo se

$$
d f_{x}(\phi(x))=\bar{\phi}(f(x))
$$

A integrabilidade de um referencial está relacionada com as funções estruturais: quando calculamos os colchetes de Lie entre os elementos do referencial $\phi$, obtemos

$$
\left[\phi_{i}, \phi_{j}\right]=\sum_{k=1}^{n} c_{i k}^{k} \phi_{k}, \quad c_{i j}^{k} \in C^{\infty}(M)
$$

em que os coeficientes $c_{i j}^{k}$ são as funções estruturais.

Seja $\theta=\left(\theta^{1}, \ldots, \theta_{n}\right)$ uma base de $T^{*} M$ que é dual de $\phi: \theta^{j}\left(\phi_{i}\right)=\delta_{i j}$. Ela é também chamada de correferencial e podemos analisar o problemas da integrabilidade do referencial olhando para o correferencial.

Proposição 3.1.1.

$$
d \theta=\sum_{i<j}-c_{i j}^{k} \theta^{i} \wedge \theta^{j}
$$

Demonstração.

$$
\begin{aligned}
d \theta^{k}\left(\phi_{i}, \phi_{j}\right) & =\phi_{i}\left(\theta^{k}\left(\phi_{j}\right)\right)-\phi_{j}\left(\theta^{k}\left(\phi_{i}\right)\right)-\theta^{k}\left[\phi_{i}, \phi_{j}\right] \\
& =-\theta^{k} \sum_{l=1}^{n} c_{i k}^{l} \phi_{l} \\
& =-c_{i k}^{k}
\end{aligned}
$$


Por outro lado,

$$
\begin{aligned}
d \theta^{k} & =\sum_{p<q} b_{p q}^{k} \theta^{p} \wedge \theta^{q} \\
\Rightarrow d \theta^{k}\left(\phi_{i}, \phi_{j}\right) & =\sum_{p<q} b_{p q}^{k} \theta^{p} \wedge \theta^{q}\left(\phi_{i}, \phi_{j}\right) \\
& =b_{i j}^{k}
\end{aligned}
$$

Logo, $b_{i j}^{\bar{E}}-c_{i j}^{k}$

Teorema 3.1.1. Um referencial é integrável se, e somente se, as funções estruturais são todas nulas.

Demonstração. Suponhamos que o referencial $\phi$ é integrável. Desse modo, $\left[\phi_{i}, \phi_{j}\right]=0$, pois é o que ocorre com o referencial padrão. Como $\left[\phi_{i}, \phi_{j}\right]=$ $c_{i j}^{k} \phi_{k}=0$, então $c_{i j}^{k}=0$, para todo $k$.

Agora assumamos que as funções estruturais são nulas. Desse modo, $\phi_{i}$ e $\phi_{j}$ comutam dois a dois. Seja

$$
\begin{gathered}
f: U \subseteq \mathbb{R}^{n} \rightarrow M \\
f\left(t_{1}, \ldots, t_{n}\right)=\Phi_{1}^{t_{1}} \circ \ldots \circ \Phi_{n}^{t_{n}}\left(x_{0}\right)
\end{gathered}
$$

onde $\Phi_{i}^{t_{i}}$ é o fluxo do campo vetorial $\phi_{i}$ e $0 \in U$. Temos que $f(0)=x_{0}$ e as derivadas parciais de $f$ em $x \in M$ nos dá $\phi_{i}(f(x))$. O teorema da função inversa garante que $f$ é difeomorfismo local definido numa vizinhança de 0 a qual pertence $x_{0}$, de modo que $\left(U, \phi_{i} \circ f\right)$ é uma carta adaptada.

Exemplo 3.1.2 (Métricas Riemennianas: $G=O(n)=\left\{A \in G L_{n}(\mathbb{R}): A A^{T}=I\right\}$ ).

Definição 3.1.5. Uma métrica riemanniana em $M$ é uma seção suave de $S^{2} T^{*} M$ que é positiva definida, ou seja, uma correspondência suave $x \mapsto$ $g_{x}$ tal que $g_{x}: T_{x} M \times T_{x} M \longrightarrow \mathbb{R}$ é um produto interno.

Uma variedade riemanniana é aquela que admite uma métrica riemanniana .

Uma métrica riemanniana em $M$ é equivalente a uma $O(n)$-estrutura. 
Seja $g^{\text {stand }}$ o produto interno usual em $\mathbb{R}^{n}: g^{\text {stand }}(u, v)=\langle u, v\rangle$. A estrutura padrão é dada por $\mathbb{R}^{n} \mathrm{com}$

$$
g^{\text {stand }}\left(\frac{\partial}{\partial x_{i}}, \frac{\partial}{\partial x_{j}}\right)=\delta_{i j}
$$

Dada um produto interno $g_{x}$ em $T_{x} M$, consideremos

$$
S_{g}=\left\{\phi \in F r(M): g\left(\phi_{i}, \phi_{j}\right)=g^{\text {stand }}\left(e_{i}, e_{j}\right)\right\}
$$

Sendo $\phi, \tilde{\phi} \in S_{g}$, consideremos $A=\tilde{\phi}^{-1} \circ \phi: \mathbb{R}^{n} \longrightarrow \mathbb{R}^{n}$, então

$$
g^{\text {stand }}\left(A\left(e_{i}\right), A\left(e_{j}\right)\right)=g\left(\phi_{i}, \phi_{j}\right)=g^{\text {stand }}\left(e_{i}, e_{j}\right)
$$

de modo que $A$ é uma transformação ortogonal e, portanto $A \in O(n)$.

Agora seja $\mathcal{S}$ uma $O(n)$-estrutura. Tomemos $\phi \in \mathcal{S}$ e definamos

$$
g_{x}(u, v)=g_{\phi(x)}^{\text {stand }}\left(\phi^{-1}(u), \phi^{-1}(v)\right)
$$

Se $\tilde{\phi} \in \mathcal{S}$, existe $A \in O(n)$ tal que: $\phi A=\tilde{\phi} \Rightarrow \phi^{-1}=A \tilde{\phi}^{-1}$. Assim

$$
\begin{aligned}
g_{\phi(x)}^{\text {stand }}\left(\phi^{-1}\left(\tilde{\phi}_{i}\right), \phi^{-1}\left(\tilde{\phi}_{j}\right)\right)= & g_{\phi(x)}^{\text {stand }}\left(G \tilde{\phi}^{-1}\left(\tilde{\phi}_{i}\right), G \tilde{\phi}^{-1}\left(\tilde{\phi}_{j}\right)\right) \\
= & g_{\phi(x)}^{\text {stand }}\left(G e_{i}, G e_{j}\right) \\
= & g_{\phi(x)}^{\text {stand }}\left(e_{i}, e_{j}\right) \\
& g_{\phi(x)}^{\text {stand }}\left(\tilde{\phi}^{-1}\left(\tilde{\phi}_{i}\right), \tilde{\phi}^{-1}\left(\tilde{\phi}_{j}\right)\right)
\end{aligned}
$$

Uma $O(n)$-estrutura é integrável se, e somente se, existe um carata local $(U, \varphi)$ em torno de cada $x \in M$ tal que

$$
g_{x}\left(\frac{\partial}{\partial \varphi_{i}}, \frac{\partial}{\partial \varphi_{j}}\right)=\delta_{i j}
$$

Observação 9. O tensor de curvatura $R(X, Y)=\nabla_{X} \circ \nabla_{Y}-\nabla_{Y} \circ \nabla_{X}-$ $\nabla_{[X, Y]}$ são ideticamente nulos em $\mathbb{R}^{n}$ ( $\nabla$ é a conexão de Levi-Civita). 
Se $\sum_{i=1}^{n} u_{i} \frac{\partial_{i}}{\partial x_{i}}$ um campo vetorial em $\mathbb{R}^{n}$, então

$$
\begin{gathered}
\nabla_{X} \nabla_{Y} \sum_{i=1}^{n} u_{i} \frac{\partial_{i}}{\partial x_{i}}=X Y \sum_{i=1}^{n} u_{i} \frac{\partial_{i}}{\partial x_{i}} \\
\sum_{i=1}^{n} u_{i} \frac{\partial_{i}}{\partial x_{i}}=Y X \sum_{i=1}^{n} u_{i} \frac{\partial_{i}}{\partial x_{i}}
\end{gathered}
$$

Portanto

$$
\left(\nabla_{X} \nabla_{Y}-\nabla_{Y} \nabla_{X}\right) \sum_{i=1}^{n} u_{i} \frac{\partial_{i}}{\partial x_{i}}=(X Y-Y X) \sum_{i=1}^{n} u_{i} \frac{\partial_{i}}{\partial x_{i}}=\nabla_{[X, Y]} u_{i} \frac{\partial_{i}}{\partial x_{i}}
$$

Assim, em $\mathbb{R}^{n}, \nabla_{X} \circ \nabla_{Y}-\nabla_{Y} \circ \nabla_{X}=\nabla_{[X, Y]}$.

Proposição 3.1.2. Uma variedade riemanniana $(M, g)$ é integrável se, e somente se, o tensor curvatura $R$ relativo ã conexão de Levi-Civita é identicamente nulo.

Demonstração. (Baseada em [Lee97])

Se a variedade é integrável, então ela é localmente equivalente a $\mathbb{R}^{n}$, de modo que o tensor de curvatura é, localmente, nulo.

Suponhamos que $R$ é identicamente nulo. Vamos mostrar, primeiramente, que $g$ admite referencial ortonormal paralelo em torno de cada ponto de $M$.

Sendo $x \in M$, podemos escolher $\left(X_{p}^{1}, \ldots, X_{p}^{n}\right)$ uma base ortonormal de $T_{x} M$. Consideremos $\left(x^{1}, \ldots, x^{n}\right)$ um sistema de coordenadas centrado em $x$ de maneira que $X_{p}^{i}=\partial / \partial x_{1}$. Podemos tomar vizinhanças de $x^{i}$ de modo que $p \in C, C$ um cubo centrado em $p$ de lado medindo $2 \varepsilon$.

Por meio do transporte paralelo, podemos deslocar cada $X_{p}^{k}$ ao longo do eixo $x^{1}$. A partir de cada ponto desse eixo, de novo por meio de do transporte paralelo, desloco agora $X_{p}^{k}$ através do eixo $x^{3}$, e assim por diante. O resultado será campos vetoriais $\left(X^{1}, \ldots, X^{n}\right)$ definidas em $C$.

O transporte paralelo preserva produto interno de modo que $\left(X^{1}, \ldots, X^{n}\right)$ formam referenciais ortonormais. Agora vamos mostrar que eles são parale- 
los. Por construção,

$$
\begin{aligned}
\nabla_{\partial / \partial x^{1}} X^{j}= & 0 \text { em }\left\{\left(x^{1}, \ldots, x^{n}\right): x^{l}=0, l \geq 2\right\} \subseteq C, \\
\nabla_{\partial / \partial x^{2} X^{j}=} & 0 \text { em }\left\{\left(x^{1}, \ldots, x^{n}\right): x^{l}=0, l \geq 3\right\} \subseteq C, \\
\nabla_{\partial / \partial x^{3}} X^{j}= & 0 \text { em }\left\{\left(x^{1}, \ldots, x^{n}\right): x^{l}=0, l \geq 4\right\} \subseteq C \\
& \quad \ldots \\
\nabla_{\partial / \partial x^{k}} X^{j}= & 0 \text { em }\left\{\left(x^{1}, \ldots, x^{n}\right): x^{l}=0, l \geq k+1\right\} \subseteq C
\end{aligned}
$$

Vamos provar por indução em $k$ que em

$$
\nabla_{\partial / \partial x^{1}} X^{j}=\ldots=\nabla_{\partial / \partial x^{k}} X^{j}=0, \operatorname{em}\left\{\left(x^{1}, \ldots, x^{n}\right): x^{l}=0, l \geq k+1\right\}(*)
$$

Quando $k=1$, isso vale por construção. Supondo que vale $(*)$, em $\left\{\left(x^{1}, \ldots, x^{n}\right)\right.$ :

$\left.x^{l}=0, l \geq k+2\right\}, \nabla_{\partial / \partial x^{k+1}} X^{j}=0$ por construção e $\nabla_{\partial / \partial x^{1}} X^{j}=\ldots=$ $\nabla_{\partial / \partial x^{k}} X^{j}=0$ em $\left\{\left(x^{1}, \ldots, x^{n}\right): x^{k+1}=0\right\}$ por hipótese. Como $\left[\partial / \partial x^{k+1}, \partial / \partial x^{i}\right]=$ $0 \mathrm{e}$

$$
\nabla_{\partial / \partial x^{k+1}}\left(\nabla_{\partial / \partial x^{i}} X^{j}\right)=\nabla_{\partial / \partial x^{i}}\left(\nabla_{\partial / \partial x^{k+1}} X^{j}\right)=0
$$

de maneira que $X^{j}$ é paralelo. Pela simetria da conexão, então $\left[X^{i}, X^{j}\right]=$ $\nabla_{\partial / \partial x^{i}} X^{j}-\nabla_{\partial / \partial x^{j}} X^{i}=0$.

Portanto, $\left(X^{1}, \ldots, X^{n}\right)$ é um referencial ortonormal para o qual existem coordenadas $\left(x^{1}, \ldots, x^{n}\right)$ para os quais $X^{j}=\partial / \partial x^{j}$ e, portanto

$$
g\left(\partial / \partial x^{i}, \partial / \partial x^{j}\right)=g\left(X^{i}, X^{j}\right)=\delta_{i j}
$$

de modo a ser $(M, g)$ integrável.

Exemplo 3.1.3 (Orientações: $G=G L_{n}^{+}(\mathbb{R})=\left\{A \in G L_{n}(\mathbb{R}): \operatorname{det}(A)>0\right\}$ ). Dois referenciais $\phi$ e $\phi^{\prime}$ de $T_{x} M$ induzem a mesma orientação se a matriz de mudança de base de $\phi^{\prime}$ para $\phi$ tem determinante positiva. Isso define uma relação de equivalência e uma orientação em $T_{x} M$ é uma classe de equivalência dessa relação.

Definição 3.1.6. Uma orientação em $M$ é uma coleção de orientações $\mathscr{O}=\left\{\mathscr{O}_{x}: x \in M\right\}$ tal que: em torno de qualquer $x_{0} \in M$ existe um aberto $U \subseteq M$ tal que se $x \in U$ e uma seção $\phi \in \Gamma_{U}(F r(M))$ tal que $\phi_{x}$ induz a orientação $\mathscr{O}_{x}$,

Uma orientação em $M$ é, desse modo, uma $G L_{n}^{+}(\mathbb{R})$-estrutura em $M$. 
$O$ modelo padrão é a orientação em $\mathbb{R}^{n}$ determinada pelo referencial canônico

$$
\left(\frac{\partial}{\partial x_{1}}, \ldots, \frac{\partial}{\partial x_{n}}\right)
$$

Nesse caso, um isomorfismo é difeomorfismo $f: M \rightarrow \bar{M}$ em que a diferencial leva referenciais orientados de $M$ em referenciais orientados de $\bar{M}$.

Uma $G L_{n}^{+}(\mathbb{R})$ ser integrável significa que em torno de cada $x \in M$ existe uma carta local $(U, \varphi)$ de maneira que a orientação que a orientação $\mathscr{O}_{x}$ do espaço tangente $T_{x} M$ é induzido por

$$
\left(\frac{\partial}{\partial \varphi_{1}}(x), \ldots, \frac{\partial}{\partial \varphi_{n}}(x)\right) .
$$

Proposição 3.1.3. Toda orientação $\mathscr{O}$ em $M$ como $G L_{n}^{+}(\mathbb{R})$-estrutura é integrável.

Demonstração. Dada uma orientação $\mathscr{O}$ em $M$ e $x_{0} \in M$, podemos escolher um referencial $\phi$ definido num aberto $U \subseteq M$ em torno de $x_{0}$ que contém o domínio de uma carta local $(V, \varphi)$, de modo que $\phi(x)$ induz a orientação $\mathscr{O}_{x}$. Podemos assumir $V$ conexo. Vamos considerar a matriz $B(x)$ da mudança de base de $\phi(x)$ para $\left(\frac{\partial}{\partial \varphi_{1}}(x), \ldots, \frac{\partial}{\partial \varphi_{n}}(x)\right)$. As entradas dessa matriz são funções suaves com respeito a $x \in V$, de modo que $\operatorname{det}(B(x))$ também é suave. Podemos adotar a carta local de modo que $\operatorname{det}\left(B\left(x_{0}\right)\right)>0$. Pela conexidade de $V$ e continuidade do determinante, $\operatorname{det}(B(x))>0$ para todo $x \in V$ de modo que $\left(\frac{\partial}{\partial \varphi_{1}}(x), \ldots, \frac{\partial}{\partial \varphi_{n}}(x)\right)$ induz a a orientação $\mathscr{O}_{x}$.

Exemplo 3.1.4 (Formas volume: $\left.G=S L_{n}(\mathbb{R})=\left\{A \in G L_{n}(\mathbb{R}): \operatorname{det}(A)=1\right\}\right)$.

Definição 3.1.7. Seja $\mu \in \Omega(M)$ uma forma que não anula-se em nenhum $x \in M$. Esta forma é uma forma volume em $M$.

Uma forma volume é $S L_{n}(\mathbb{R})$-estrutura. A $S L_{n}(\mathbb{R})$-estrutura padrão é $\mathbb{R}^{n}$ com $\mu^{\text {stand }}=d x_{1} \wedge \ldots \wedge d x_{n}$

Seja $\mathcal{S}_{\mu}=\left\{\phi \in \operatorname{Fr}(M): \phi^{*} \mu=\mu_{\text {stand }}\right\}$. Então

$$
\mu\left(\phi_{1}\left(e_{1}\right), \ldots, \phi_{n}\left(e_{n}\right)\right)=1=\mu^{\text {stand }}\left(e_{1}, \ldots, e_{n}\right)
$$


Tomemos $\phi, \tilde{\phi} \in \mathcal{S}_{\mu}$. Vamos provar que a composta $\tilde{\phi}_{x}^{-1} \circ \phi_{x}: \mathbb{R}^{n} \rightarrow \mathbb{R}^{n}$ é um elemento de $S L_{n}(\mathbb{R})$ :

$$
\begin{aligned}
\mu^{\text {stand }}\left(\tilde{\phi}_{x}^{-1} \circ \phi_{x}\left(e_{1}\right), \ldots, \tilde{\phi}_{x}^{-1} \phi_{x}\left(e_{n}\right)\right) & =\operatorname{det}\left(\tilde{\phi}_{x}^{-1} \circ \phi_{x}\right) \mu^{s t a n d}\left(e 1, \ldots, e_{n}\right) \\
& =\operatorname{det}\left(\tilde{\phi}_{x}^{-1} \circ \phi_{x}\right) \cdot 1 \\
& =\operatorname{det}\left(\tilde{\phi}_{x}^{-1} \circ \phi_{x}\right)
\end{aligned}
$$

Mas

$$
\begin{aligned}
\phi^{*} \mu=\mu_{\text {satnd }} & \Rightarrow \mu \circ \phi=\mu^{\text {stand }}, \mu \circ \tilde{\phi}=\mu_{\text {stand }} \\
& \Rightarrow \mu \circ \phi=\mu^{\text {stand }}, \mu=\mu_{\text {stand }} \circ \tilde{\phi}^{-1} \\
& \Rightarrow \mu^{\text {stand }} \circ\left(\tilde{\phi}^{-1} \circ \phi\right)=\mu_{\text {stand }}
\end{aligned}
$$

Portanto

$\operatorname{det}\left(\tilde{\phi}_{x}^{-1} \circ \phi_{x}\right)=\mu^{\text {stand }}\left(\tilde{\phi}_{x}^{-1} \circ \phi_{x}\left(e_{1}\right), \ldots, \tilde{\phi}_{x}^{-1} \phi_{x}\left(e_{n}\right)\right)=\mu^{\text {stand }}\left(e_{1}, \ldots, e_{n}\right)=1$

Agora, dada $\mathcal{S}$ uma $S L_{n}(\mathbb{R})$-estrutura, para $\phi \in \mathcal{S}$, definamos

$$
\mu_{x}\left(x_{1}, \ldots, x_{n}\right)=\mu_{\phi^{-1}(x)}^{\text {stand }}\left(\phi^{-1}\left(x_{1}\right), \ldots, \phi^{-1}\left(x_{n}\right)\right)
$$

Dado $\tilde{\phi} \in \mathcal{S}$, existe $G \in S L_{n}(\mathbb{R})$ tal que: $\phi G=\tilde{\phi}$. Dessa forma,

$$
\phi G=\tilde{\phi} \Rightarrow \phi^{-1}=G \tilde{\phi}^{-1} .
$$

Assim

$$
\begin{aligned}
\mu_{\phi^{-1}(x)}^{\text {stand }}\left(\phi^{-1}\left(x_{1}\right), \ldots, \phi^{-1}\left(x_{n}\right)\right) & =\mu_{\tilde{\phi}^{-1}(x)}^{\text {stand }}\left(G \tilde{\phi}^{-1}\left(x_{1}\right), \ldots, G \tilde{\phi}^{-1}\left(x_{n}\right)\right) \\
& =\operatorname{det}(G) \mu_{\tilde{\phi}^{-1}(x)}^{\text {stand }}\left(\tilde{\phi}^{-1}\left(x_{1}\right), \ldots, \tilde{\phi}^{-1}\left(x_{n}\right)\right) \\
& =\mu_{\tilde{\phi}^{-1}(x)}^{\text {stand }}\left(\tilde{\phi}^{-1}\left(x_{1}\right), \ldots, \tilde{\phi}^{-1}\left(x_{n}\right)\right)
\end{aligned}
$$

Um isomorfismo aqui será um difeomorfismo $f: M \rightarrow \bar{M}$ em que $f^{*}(\mu)=\bar{\mu}$.

Uma $S L_{n}(\mathbb{R})$-estrutura é integrável se em torno de cada $x \in M$ existe 
uma carta local $(U, \varphi)$ de modo que

$$
\mu=d \varphi_{1} \wedge \ldots \wedge \varphi_{n}
$$

Proposição 3.1.4. Toda forma volume $\mu$ em $M$ é integrável.

Demonstração. Seja $(U, \varphi)$ uma carta local contendo $x \in M$. Então:

$$
\mu=f d \varphi_{1} \wedge \ldots \wedge \varphi_{n}
$$

onde $f \in C^{\infty}(U)$. Definamos numa vizinhança $U^{\prime} \subseteq U$ de $x$ uma função $\eta: U \rightarrow \mathbb{R}$ tal que $\frac{\partial \eta}{\partial \varphi_{1}}=f$ e $\frac{\partial \eta}{\partial \varphi_{i}}=0, j>1$. Dessa forma $d \eta=f d \varphi_{1}$, de modo que usando as coordenadas $\left(\eta, \varphi_{2}, \ldots, \varphi_{n}\right)$ teremos

$$
\mu=d \eta \wedge \ldots \wedge \varphi_{n}
$$

Exemplo 3.1.5 (Folheações: $G=G L(p, n-p)=$

$\left.\left\{A \in G L_{n}(\mathbb{R}): A=\left[\begin{array}{cc}M & N \\ 0 & P\end{array}\right], M \in G L_{p}(\mathbb{R}), P \in G L_{n-p}(\mathbb{R})\right\}\right)$.

Definição 3.1.8. Um subfibrado vetorial $F \subseteq T M$ de posto $p$ é uma distribuição p-dimensional da variedade $M$.

$A$ escolha de uma p-distribuição é o mesmo que escolher uma $G=$ $G L(p, n-p)$-estrutura.

Definição 3.1.9. Uma distribuição $F \subseteq T M$ é involutiva se

$$
[X, Y] \in \Gamma(F), X, Y \in \Gamma(F)
$$

Definição 3.1.10. Uma folheação p-dimensional é uma distribuição $p$ dimensional que é involutiva.

O modelo padrão é $\mathbb{R}^{n}$ com a folheação $F^{\text {stand }}=\operatorname{span}\left\{\frac{\partial}{\partial x_{i}}\right\}_{i=1}^{p}$

Uma $G=G L(p, n-p)$-estrutura é integrável se em torno de todo $x \in M$ existe uma carta local $(U, \varphi)$ de modo que

$$
F_{x}=\operatorname{span}\left\{\frac{\partial}{\partial \varphi_{1}}(x), \ldots, \frac{\partial}{\partial \varphi_{p}}(x)\right\}
$$


Teorema 3.1.2 (Frobenius). Seja Fum distribuição k-dimensional em $M$. São equivalentes:

1. F é involutiva.

2. $F$ como $G=G L(p, n-p)$-estrutura é integrável.

Demonstração. (Baseda em [Lee13]) 0 .

Se a distribuição $F$ é integrável, então ela será involutiva pois $\left[\frac{\partial}{\partial \varphi_{i}}, \frac{\partial}{\partial \varphi_{j}}\right]=$

Agora suponhamos que $F$ é involutiva. Seja $x \in M$ e $(U, \varphi)$ uma carta local em torno de $x$. Se necessário, podemos reordenar as coordenada de modo que $F_{x}$ seja o espaço complementar de $T_{x} M$ gerado por $\frac{\partial}{\partial \varphi_{p+1}}(x), \ldots, \frac{\partial}{\partial \varphi_{n}}(x)$.

Consideremos $\pi: U \rightarrow \mathbb{R}^{n}$ a projeção nas primeiras $p$ coordenadas. A diferencial, quando restrita a $F_{x}$, induz um isomorfismo

$$
(d \pi)_{x}: F_{x} \rightarrow T_{\pi(x)} \mathbb{R}^{p}
$$

Sejam $V_{i, x}=\left(\left.(d \pi)_{x}\right|_{F_{x}}\right)^{-1}\left(\frac{\partial}{\partial x_{i}}\right)(\pi(x)), i=1, \ldots, p$. Dessa forma,

$$
d \pi_{x}\left(\left[V_{i}, V_{j}\right]_{x}\right)=\left[\frac{\partial}{\partial x_{i}}, \frac{\partial}{\partial x_{i}}\right]_{x}=0
$$

Portanto, existe um referencial de $F_{x}$ difeomorfo ao referencial padrão.

Exemplo 3.1.6 (Estruturas complexas: $G=G L_{n}(\mathbb{C})$ ). Aqui faremos a identificação $\mathbb{C}^{k} \simeq \mathbb{R}^{2 n}$ através de

$$
\left(x_{1}+i y_{1}, \ldots, x_{n}+i y_{n}\right) \mapsto\left(x_{1}, \ldots, x_{n}, y_{1}, \ldots, y_{n}\right)
$$

Definição 3.1.11. Uma estrutura quase complexa em uma variedade $M$ é uma aplicação $J: T M \rightarrow T M$ que é um morfismo de fibrados vetoriais com a seguinte característica:

$$
J^{2}=-I d
$$

Estruturas quase complexas são $G L_{n}(\mathbb{C})$-estruturas. 
O modelo padrão é desta vez $\mathbb{C}^{n} \simeq \mathbb{R}^{2 n}$, coma a estrutura quase complexa

$$
\begin{gathered}
J^{\text {stand }}\left(\frac{\partial}{\partial x_{n}}\right)=\frac{\partial}{\partial y_{n}} \\
J^{\text {stand }}\left(\frac{\partial}{\partial y_{n}}\right)=-\frac{\partial}{\partial x_{n}} .
\end{gathered}
$$

A integrabilidade de uma $G L_{n}(\mathbb{C})$-estrutura significa encontrar em trono de cada $x \in M$ uma carta coordena $(U, \varphi), \varphi=\left(x_{1}, \ldots, x_{n}, y_{1}, \ldots, y_{n}\right)$ e

$$
\begin{gathered}
J\left(\frac{\partial}{\partial x_{n}}\right)=\frac{\partial}{\partial y_{n}} \\
J\left(\frac{\partial}{\partial y_{n}}\right)=-\frac{\partial}{\partial x_{n}} .
\end{gathered}
$$

Definição 3.1.12. A aplicação

$$
\begin{gathered}
N_{J}: \mathfrak{X}(M) \times \mathfrak{X}(M) \rightarrow \mathfrak{X}(M) \\
N_{J}(X, Y)=[X, Y]+J([J X, Y]+[X, J Y])-[J X, J Y]
\end{gathered}
$$

é o tensor de Nijenhuis da estrutura quase complexa J.

\section{Proposição 3.1.5.}

$$
N_{J} \in \Gamma\left(\Lambda^{2} T^{*} M \otimes T M\right)
$$

Demonstração. $N_{J}$ é antissimétrica:

$$
\begin{aligned}
N_{J}(X, Y) & =[X, Y]+J([J X, Y]+[X, J Y])-[J X, J Y] \\
& =-[Y, X]-J([Y, J X]-[J Y, X])+[J Y, J X] \\
& =-([Y, X]+J([Y, J X]+[J Y, X])-[J Y, J X]) \\
& =-N_{J}(Y, X)
\end{aligned}
$$


Vamos provar que $N_{J}$ é tensorial. Sejam $X, Y \in \mathfrak{X}(M)$ e $f \in C^{\infty}(M)$. Então

$$
\begin{aligned}
N_{J}(f X, Y)= & {[f X, Y]+J[J(f X), Y]+J[f X, J Y]-[J f X, J Y] } \\
= & f[X, Y]-Y(f X)+J[f J X, Y]+J f[X, J Y]-J^{2}(Y f) X-f[J X, J Y]+J(Y f) J X \\
= & f[X, Y]-Y(f X)+J f[J X, Y]-J(Y f) X \\
& +J f[X, J Y]+(Y f) X-f[J X, J Y]+J(Y f) J X \\
= & f[X, Y]+f J[X, J Y]+f J[X, J Y]-f[J X, J Y] \\
= & f N_{J}
\end{aligned}
$$

A seguir vem um resultado muito profundo sobre a integrabilidade de estruturas que é o Teorema de Newlander-Niremberg. Trata-se de um teorema não trivial cuja demonstração é muito trabalhosa e não será feita, sendo indicada uma referência.

Teorema 3.1.3 (Newlander-Niremberg). Se J é uma estrutura quase complexa em $M$, então são equivalentes:

1. $N_{J} \equiv 0$

2. A $G L_{k}(\mathbb{C})$-estrutura $J$ é integrável.

Demonstração. Ver em[NN57] ou em [Dem12], página 399.

Exemplo 3.1.7 (Estruturas simpléticas: $G=S p_{2 n}(\mathbb{R})=\left\{A \in G L_{2 n}(\mathbb{R}): A^{T} J^{\text {stand }} A=J^{\text {stand }}\right\}$, $\left.J^{\text {stand }}=\left[\begin{array}{cc}0 & -I_{n} \\ I_{n} & 0\end{array}\right]\right)$.

Definição 3.1.13. Uma estrutura quase simplética $\omega$ em $M$ é

$$
\omega \in \Omega^{2}(M)
$$

em que $\omega_{x}$, para todo $x \in M$ é não degenerada:

$$
\omega_{x}(u, v)=0, \forall v \Longleftrightarrow u=0
$$

Definição 3.1.14. Uma estrutura quase simplética wserá dita simplética se $\omega$ é fechada $(d \omega=0)$ 
Um isomorfismo, nesse caso, é um difeomorfismo $f: M \rightarrow \bar{M}$ tal que $f^{*} \bar{\omega}=\omega$, em que $\omega$ e $\bar{\omega}$ são estruturas quase simpléticas em $M$ e $\bar{M}$, respectivamente.

Uma estrutura quase simplética é uma $S p_{2 n}(\mathbb{R})$-estrutura em $M$ e o modelo padrão é $\mathbb{R}^{2 n}$ com seus elementos denotados por $\left(x_{1}, \ldots, x_{n}, y_{1}, \ldots, y_{n}\right)$ e

$$
\omega^{\text {stand }}=d x_{1} \wedge d y_{1}+\ldots+d x_{n} \wedge d y_{n}
$$

Uma $S p_{2 n}(\mathbb{R})$-estrutura é integrável se , para cada $x \in M$, existe uma carta local $\left(U, \varphi=\left(x_{1}, \ldots, x_{n}, y_{1}, \ldots, y_{n}\right)\right)$ contendo $x$ tal que

$$
\omega=d x_{1} \wedge d y_{1}+\ldots+d x_{n} \wedge d y_{n}
$$

Lema 3.1.1 (Truque de Moser Local). Seja $\left\{\omega_{t}\right\}_{0 \leq t \leq 1}$ uma família de formas simpléticas diferenciáveis em $t$. Então existe uma vizinhança $U$ de $p \in M$ tal que existe uma função $g_{t}: U \rightarrow U$ de maneira que $g_{t}^{*} \omega_{t}=\omega_{0} e$ $g_{0}^{*}=i d$

Demonstração. Apêndice, Teorema A.5.1.

Teorema 3.1.4 (Darboux). Se $\omega$ é uma estrutura quase simplética em $M$. Então, são equivalentes

1. $\omega$ é simplética

2. $\omega$ é integrável.

Demonstração. (Retirada de [Les14])

Vamos provar que se $\omega$ é uma forma simplética em $M$, então existe uma vizinhança em torno de cada ponto de $M$ de modo que existem $\left(x_{1}, \ldots, x_{n}, y_{1}, \ldots, y_{n}\right)$ coordenadas locais para as quais $\omega=d x_{1} \wedge d y_{1}+$ $\ldots+d x_{n} \wedge d y_{n}$.

Sejam $\left(x_{1}, \ldots, x_{n}, y_{1}, \ldots, y_{n}\right)$ coordenadas locais em $M$ e $\omega_{t}=\omega_{0}+t(\omega-$ $\left.\omega_{0}\right), t \in[0,1]$ uma família de 2 -formas com $\omega_{0}=d x_{1} \wedge d y_{1}+\ldots+d x_{n} \wedge d y_{n}$. Cada $\omega_{t}$ é fechada: $\operatorname{Em} x \in M, \omega_{t}(x)=\omega_{0}(x)=\omega(x)$

Por continuidade pode-se determinar uma vizinhança $V$ de p de modo que $\omega_{t}(p)$ não é degenerada. Pelo lema de Moser, existe uma vizinhança 
$U \subseteq V$ de modo que há uma função $g_{t}: U \rightarrow U$ como no lema. Desse modo:

$$
\begin{aligned}
g_{t}^{*} \omega_{t}=\omega_{0} & \Rightarrow \frac{d}{d t} g_{t}^{*} \omega_{t}=0 \\
& \Rightarrow g_{t}^{*}\left(\frac{d}{d t} \omega_{t}+\mathcal{L}_{X_{t}} \omega_{t}\right)=0 \\
& \Rightarrow g_{t}^{*}\left(\frac{d}{d t} \omega_{t}+d i_{X_{t}} \omega_{t}\right)=0 \\
& \Rightarrow d i_{X_{t}} \omega_{t}=-\frac{d}{d t} \omega_{t}
\end{aligned}
$$

Além disso

$$
\frac{d}{d t} \omega_{t} \text { exata } \Rightarrow d i_{X_{t}} \omega_{t}=d \eta_{t}, \text { numa vizinhança de } \mathrm{p}, \eta_{t} \in \Omega^{1}(M)
$$

Portanto, $i_{X_{t}} \omega_{t}=\eta_{t}$ já que $\omega_{t}$ é não-degenerada. esta equação tem solução, que determina um único campo vetorial $X_{t}$. Desse modo, existe $g_{1}$ tal que $g_{1}^{*} \omega=\omega_{0}$.

O campo vetorial $X_{t}$ gera famílias de um-parâmetros de difeomorfismos $\left\{g_{t}\right\}_{0 \leq t \leq 1}$ de modo que se pode mudar as coordenadas e obter $\omega=d x_{1} \wedge$ $d y_{1}+\ldots+d x_{n} \wedge d y_{n}$.

\subsection{Conexões compatíveis}

\subsubsection{Compatibilidade entre conexões e $G$-estruturas}

Definição 3.2.1. Uma conexão $\nabla: \mathfrak{X}(M) \times \mathfrak{X}(M) \rightarrow \mathfrak{X}(M)$ é compatível com a G-estrutura $\mathcal{S}$ se, para toda curva $\gamma:[0,1] \rightarrow M$, o transporte paralelo ao longo de $\gamma$ com respeito $a \nabla$

$$
P_{\gamma}^{t_{0}, t_{1}}: T_{\gamma\left(t_{0}\right)} M \longrightarrow T_{\gamma\left(t_{1}\right)} M
$$

leva $G$-referenciais em G-referenciais.

Proposição 3.2.1. Seja $\nabla$ uma conexão e $\mathcal{S}$ um G-estrutura. Então são equivalentes:

1. $\nabla$ é compativel com $\mathcal{S}$ 
2. Se $\phi$ é um referencial local em $U \subseteq M$ tal que $\phi \in \mathcal{S}$ então a matriz da conexão $\mu(\nabla, \phi) \in \Omega^{1}(U, \mathfrak{g})$ (ver observação 8)

3. A conexão de fibrados principais em $\operatorname{Fr}(M)$ representada pela 1-forma de conexão $\mu \in \Omega\left(F r(M), g l_{r}\right)$ é tal que $\left.\mu\right|_{\mathcal{S}} \in \Omega^{1}(\mathcal{S}, \mathfrak{g})$.

Demonstração. Vamos provar a equivalência entre (i) e (ii).

Primeiro provaremos a implicação direta: o cálculo do valor de $\mu$ aplicado a um vetor tangente $u^{\prime}(0)$ equivale a levar $u(t)$ a $T_{\gamma\left(t_{0}\right)} M$ através do transporte paralelo e depois tomar a dua derivada com relação a $t$ quando $t=0$. Assim, se o transporte paralelo preserva $\mathcal{S}$ e $u^{\prime}(0)$ é tangente a $\mathcal{S}$ e, logo, o processo resulta num elemento de $\mathcal{S}$, originando um elemento tangente a $\mathcal{S}$, ou seja, pertencente a $\mathfrak{g}$.

Agora, provemos a recíproca: $\left.\mu\right|_{\mathcal{S}}$ é uma conexão em $\mathcal{S}$ e para curvas em $\mathcal{S} \subseteq \operatorname{Fr}(M)$, a horizontalidade de $\mu$ e $\left.\mu\right|_{\mathcal{S}}$ coincidem. Portanto, o transporte paralelo relativo a $\mu$ aplicado a elementos de $\mathcal{S}$ coincide com o transporte paralelo relativo a $\left.\mu\right|_{\mathcal{S}}$ de modo a preservar elementos de $\mathcal{S}$.

Vamos provar a equivalência entre (ii) e (iii): $\mu(\nabla, \phi)$ aplicada a $u \in T_{x} M$ coincide $\operatorname{com} \mu\left(h_{\phi_{x}}(u)\right)$.

\subsection{2 espaço das conexões compatíveis}

O espaço das conexões $\nabla$ em $T M$ é um espaço afim modelado por $\Omega^{2}(M, \operatorname{End}(T M))$. Isso se explica pelo fato de o espaço das conexões não é um um espaço vetorial, mas duas conexões $\nabla^{1}, \nabla^{2}$ originam o elemento

$$
\begin{gathered}
\lambda\left(\nabla^{1}, \nabla^{2}\right)=\nabla^{1}-\nabla^{2} \in \Omega^{1}(E, \operatorname{End}(T M)), \\
\nabla^{1}-\nabla^{2}: \Gamma(T M) \times \Gamma(T M) \longrightarrow \Gamma(T M)
\end{gathered}
$$

e pela seguinte proposição:

Proposição 3.2.2. Dada $\nabla$ uma conexão compativel com a $G$-estrutura $\mathcal{S}$, então é possível construir uma conexão $\nabla^{\lambda}$ ainda compatível com $\mathcal{S}$, para toda $\lambda \in \Omega^{1}\left(M, \mathfrak{g}_{\mathcal{S}}\right)$.

Demonstração. Dada uma conexão $\nabla$ e $\lambda \in \Omega^{1}(M, E n d(T M))$, podemos obter uma nova conexão

$$
\nabla_{\lambda}: \Gamma(T M) \times \Gamma(T M) \longrightarrow \Gamma(T M)
$$




$$
\nabla_{X}^{\lambda} Y=\nabla_{X} Y+\lambda(X)(Y) .
$$

A escolha de $\nabla$ da origem a uma bijeção $\lambda \mapsto \nabla^{\lambda}$ entre $\Omega^{1}(M, \operatorname{End}(T M))$ e o espaço das conexões.

Lema 3.2.1. Seja $\nabla$ uma conexão compativel com a G-estrutura $\mathcal{S}$ e $\xi \in$ $\Omega^{1}\left(M, \mathfrak{g}_{\mathcal{S}}\right)$, então

$$
T_{\nabla \xi}=T_{\nabla}+\mathcal{F}(\xi)
$$

onde $T_{\nabla \xi}$ é a torção relativa à conexão $\nabla^{\xi}$

Demonstração.

$$
\begin{aligned}
T_{\nabla^{\xi}}(X, Y) & =\nabla_{X}^{\xi} Y-\nabla_{Y}^{\xi} X-[X, Y] \\
& =\nabla_{X} Y+\xi(X)(Y)-\nabla_{Y} X-\xi(Y)(X)-[X, Y] \\
& =T_{\nabla}(X, Y)+\xi(X)(Y)-\xi(Y)(X) \\
& =T_{\nabla}(X, Y)+\mathcal{F}(\xi)(X, Y)
\end{aligned}
$$

Observação 10. A torção $T_{\nabla}$ pode ser vista como uma aplicação

$$
\bar{T}_{\nabla} \mathcal{S} \longrightarrow \operatorname{Hom}\left(\Lambda^{2} V, V\right)
$$

Se $\phi \in \mathcal{S}$ então

$$
\bar{T}_{\nabla}(\phi)(u, v)=\phi^{-1} T \nabla(\phi(u), \phi(v))
$$

\subsection{Curvatura e torção de G-estruturas}

\subsubsection{Torção intrínseca}

Vamos considerar $V=\mathbb{R}^{n}$ e $\mathfrak{g} \subseteq g l(V)$. Consideremos a seguinte aplicação linear:

$$
\begin{gathered}
\mathcal{F}: \operatorname{Hom}(V, \mathfrak{g}) \rightarrow \operatorname{Hom}\left(\Lambda^{2} V, V\right) \\
\mathcal{F}(f)(u, v)=f(u)(v)-f(v)(u)
\end{gathered}
$$

Definição 3.3.1. O primeiro prolongamento de $\mathfrak{g}$

$$
\mathfrak{g}^{(1)}:=\operatorname{ker}(\mathcal{F})=\{f: V \rightarrow \mathfrak{g} \mid f(u)(v)=f(v)(u)\}
$$


Definição 3.3.2. O espaço de torção de $\mathfrak{g}$ é o quociente.

$$
\mathcal{T}(\mathfrak{g}):=\frac{\operatorname{Hom}\left(\Lambda^{2} V, V\right)}{\mathcal{F}(\operatorname{Hom}(V, \mathfrak{g}))}
$$

Proposição 3.3.1. Seja $\nabla$ uma conexão compatível com $\mathcal{S}$. Se sua torção é $T_{\nabla}$, a composição com a projeção sobre $\mathcal{T}(\mathfrak{g})$, o resultado

$$
\mathfrak{T}: \mathcal{S} \longrightarrow \mathcal{T}(\mathfrak{g})
$$

não depende da escolha da conexão.

Demonstração. Sejam $\nabla^{1}$ e $\nabla^{2}$ duas conexões compatíveis com $\mathcal{S}$. Assim:

$$
\begin{aligned}
T_{\nabla^{1}}-T_{\nabla^{2}} & =\nabla_{X}^{1} Y-\nabla_{Y}^{1} X-[X, Y]-\nabla_{X}^{2} Y+\nabla_{Y}^{2} X+[X, Y] \\
& =\nabla_{X}^{1} Y-\nabla_{X}^{2} Y-\left(\nabla_{Y}^{1} X-\nabla_{Y}^{2} X\right) \\
& =\left(\nabla^{1}-\nabla^{2}\right)_{X} Y-\left(\nabla^{1}-\nabla^{2}\right)_{Y} X .
\end{aligned}
$$

Assim, $T_{\nabla^{1}}-T_{\nabla^{2}} \in \mathcal{F}(\operatorname{Hom}(V, \mathfrak{g}))$ e assim $\left[T_{\nabla^{1}}\right]=\left[T_{\nabla^{2}}\right]$, de modo que o resultado da composição não depende da escolha da conexão.

Definição 3.3.3. A aplicação T da proposição anterior é chamada de torção intrínseca de $\mathcal{S}$.

Teorema 3.3.1. Se a G-estrutura $\mathcal{S}$ é integrável, então a torção intrínseca Té nula.

Demonstração. Se a $G$-estrutura $\mathcal{S}$ é integrável, então ela é, localmente, equivalente a $G$-estrutura canônica flat. Portanto, a torção com respeito à conexão plana canônica é nula de modo que o resultado da composição acima será nulo.

Proposição 3.3.2. Seja $G \subseteq G L_{n}(\mathbb{R})$ e $\mathcal{S}$ uma $G$-estrutura arbitrária em M. Então

1. Se $\mathfrak{g}^{(1)}=0$ então $M$ admite no máximo uma conexão livre de torção compativel com $\mathcal{S}$.

2. $S e \mathcal{T}(\mathfrak{g})=0$ então $M$ admite, ao menos, uma conexão livre de torção compativel com $\mathcal{S}$. 
Demonstração. $\quad$ 1. Suponhamos que $\mathfrak{g}^{(1)}=\operatorname{ker} \mathcal{F}=\{0\}$, e $T_{\nabla}=0$. Seja $\nabla^{\prime}$ outra conexão livre de torção.

Então

$$
\left(T_{\nabla}-T_{\nabla^{\prime}}\right)(X, Y)=0
$$

Logo,

$$
\begin{aligned}
\left(\nabla-\nabla^{\prime}\right)_{X} Y-\left(\nabla-\nabla^{\prime}\right)_{Y} X=0 & \Rightarrow \nabla-\nabla^{\prime} \in k e r \mathcal{F} \\
& \Rightarrow \nabla-\nabla^{\prime}=0 \\
& \Rightarrow \nabla=\nabla^{\prime}
\end{aligned}
$$

2. Seja $\nabla$ uma conexão compatível com $\mathcal{S}$. Como $\mathcal{T}(\mathfrak{g})=0$ então $T_{\nabla} \in$ $\operatorname{Im}(\mathcal{F})$, ou seja, $T_{\nabla}=\mathcal{F}(\lambda)$. Então, $T_{\nabla}-\mathcal{F}(\lambda)$ é a torção desejada.

\subsection{Equações estruturais, curvatura e torção}

\subsubsection{Forma tautológica}

Definição 3.4.1. Seja $G$ um subgrupo de Lie de $G L_{n}(\mathbb{R})$ e $\mathcal{S}$ uma $G$ estrutura em uma variedade $M$. A forma tautológica é a 1-forma $\theta_{\mathcal{S}} \in$ $\Omega\left(\mathcal{S}, \mathbb{R}^{n}\right)$, em que, sendo $\phi \in \mathcal{S}$ e $X_{\phi} \in T_{\phi} \mathcal{S}$, então

$$
\theta_{\mathcal{S}}\left(X_{\phi}\right):=\phi^{-1}\left(\left(d_{\phi} \pi\right)\left(X_{\phi}\right)\right)
$$

Definição 3.4.2. Seja $V$ um espaço vetorial e $P$ um G-fibrado principal. A forma $\omega \in \Omega(P, V)$ é estritamente horizontal se $\operatorname{ker}\left(\omega_{p}\right)=\operatorname{ker}\left(d_{p} \pi\right)$.

Proposição 3.4.1. A forma tautológica é estritamente horizontal.

Demonstração. Seja $X_{\phi} \in T_{\phi} \mathcal{S}$. Se $X_{\phi} \in \operatorname{ker}\left(\theta_{\mathcal{S}}\right)$, então:

$$
\phi^{-1}\left(\left(d_{\phi} \pi\right)\left(X_{\phi}\right)\right)=0
$$

Como $\phi$ é um isomorfismo então

$$
\left(d_{\phi} \pi\right)\left(X_{\phi}\right)=0
$$


Dessa forma, $X_{\phi}$ é um vetor vertical. $\operatorname{Logo}, \operatorname{ker}\left(\theta_{\mathcal{S}}\right) \subseteq \operatorname{ker}\left(d_{\phi} \pi\right)$.

Se $X_{\phi} \in \operatorname{ker}\left(d_{\phi} \pi\right)$, então $\left(d_{\phi} \pi\right)\left(X_{\phi}\right)=0$. Portanto, $\phi^{-1}\left(\left(d_{\phi} \pi\right)\left(X_{\phi}\right)\right)=0$ e $X_{\phi} \in \operatorname{ker}\left(\theta_{\mathcal{S}}\right)$. Desse modo $\operatorname{ker}\left(d_{\phi} \pi\right) \subseteq \operatorname{ker}\left(\theta_{\mathcal{S}}\right)$

Proposição 3.4.2. Seja $G \subseteq G L_{n}(\mathbb{R})$ um subgrupo de Lie fechado, $\pi: P \rightarrow$ $M$ é um $G$-fibrado principal com $M$ uma variedade de dimensão n, e $\tau \in$ $\Omega\left(P, \mathbb{R}^{n}\right)$ uma 1-forma $G$-equivariante e estritamente horizontal. Então, se $\theta$ é a forma tautológica em $\operatorname{Fr}(M)$, existe uma único mergulho G-equivariante $F: P \rightarrow F r(M)$ de modo que $F^{*} \theta=\tau$.

Demonstração. Consideremos $\tau: T_{p} P \rightarrow \mathbb{R}^{n}$ uma 1-forma $G$-invariante e estritamente horizontal. Seja $x=\pi(p) \in M$. Definamos a seguinte função

$$
\begin{gathered}
F(p): \mathbb{R}^{n} \rightarrow T_{x} M \\
F(p)(v)=d_{p} \pi\left(\left.\tau\right|_{H_{p}} ^{-1}(v)\right)
\end{gathered}
$$

em que $H$ é uma conexão em $P$.

Vamos provar que:

1. a função $F$ não depende da escola da conexão: sejam $H$ e $H^{\prime}$ duas conexões em $P$ e $v \in \mathbb{R}^{n}$. Então

$$
\left.\tau\right|_{H_{p}} ^{-1}(v)-\left.\tau\right|_{H_{p}^{\prime}} ^{-1}(v)=w
$$

que é um vetor vertical, pois

$$
\tau\left(\left.\tau^{-1}\right|_{H_{p}}(v)-\left.\tau^{-1}\right|_{H_{p}^{\prime}}(v)\right)=v-v=0
$$

e $\tau$ é estritamente horizontal. Logo,

$$
F(p)(v)-F^{\prime}(p)(v)=d_{p} \pi(w)=0 \Rightarrow F(p)=F(p)
$$


2. F é G-equivariante:

$$
\begin{aligned}
F(p g)(v) & =d_{p g} \pi\left(\left.\tau^{-1}\right|_{H_{p g}}(v)\right) \\
& =d_{p g} \pi\left(d_{p} R_{g}\left(\left.\tau^{-1}\right|_{H_{p}}(g v)\right)\right) \\
& =d_{p g} \pi\left(\left.\tau^{-1}\right|_{H_{p}}(g v)\right) \\
& =F(p)(g v) \\
& =(F(p) g)(v)
\end{aligned}
$$

3. F é um mergulho: $F$ é um morfismo de fibrados cobrindo a identidade, com respeito à inclusão $G \hookrightarrow G L_{n}$. Como $G$ é um subgrupo fechado, então segue que $F$ é um mergulho.

Agora, vamos provar que $F^{*} \theta=\tau$, onde $\theta$ é a forma tautológica. Seja $X \in T_{p} P$. Então

$$
\begin{aligned}
\left(F^{*} \theta\right)_{p}(X) & =\theta_{p}\left(d_{p} F(X)\right) \\
& =F(p)^{-1}\left(d_{F(p)} \pi d_{p} F(X)\right) \\
& =F(p)^{-1}\left(d_{p}(\pi \circ F)(X)\right) \\
& =F(p)^{-1}\left(d_{p} \pi(X)\right) \\
& =\left(\left(\left.d_{p} \pi \circ \tau^{-1}\right|_{H_{p}}\right)\right)^{-1}\left(d_{p} \pi(X)\right) \\
& =\tau_{p}(X)
\end{aligned}
$$

\subsubsection{Torção e equações estruturais.}

Definição 3.4.3. Seja $\omega$ uma conexão no $G$-fibrado principal $\mathcal{S}$, advindo de uma G-estrutura. A torção de $\omega$ é a 2-forma $T_{\omega} \in \Omega^{2}\left(\mathcal{S}, \mathbb{R}^{n}\right)$ tal que

$$
T_{\omega}(h(X), h(Y))=d \theta(h(X), h(Y))
$$

onde $h$ é o levantamento horizontal associado a $\omega$.

Agora, vamos fixar $\mathcal{S}$ uma $G$-estrutura e uma conexão com ela compatível. Considere a correspondente forma de conexão $\omega \in \Omega^{1}(\mathcal{S}, \mathfrak{g})$ e a curvatura $K_{\omega}$ e a torção $T_{\omega}$ com respeito a $\omega$. 
Primeiramente, vamos estabelecer o significado de algumas notações. Seja $V=\mathbb{R}^{n}$ e $P=\mathcal{S}$.

1. Seja $\theta \in \Omega^{1}(P, V)$ a forma tautológica. Então, $\theta \wedge \theta \in \Omega^{2}(P, V \wedge V)$ com

$$
\theta \wedge \theta(X, Y)=\theta(X) \wedge \theta(Y)
$$

2. Temos que $T_{\omega} \in C^{\infty}\left(S, \Lambda^{2} V, V\right)$. Então $T_{\omega}(\theta \wedge \theta) \in \Omega(P, V)$, com

$$
T_{\omega}(\theta \wedge \theta)_{p}\left(X_{p}, Y_{p}\right)=T_{\omega}\left(\theta_{p}\left(X_{p}\right) \wedge \theta_{p}\left(Y_{p}\right)\right)
$$

3. Sabemos que $K \in C^{\infty}\left(\operatorname{Hom}\left(\Lambda^{2}(V), \mathfrak{g}\right)\right)$. Então, $K(\theta \wedge \theta) \in \Omega(P, \mathfrak{g})$, com

$$
K(\theta \wedge \theta)_{p}\left(X_{p}, Y_{p}\right)=K\left(\theta_{p}\left(X_{p}\right) \wedge \theta_{p}\left(Y_{p}\right)\right)
$$

4. $\omega \in \Omega^{1}(P, \mathfrak{g})$. O colchete $[\omega, \omega] \in \Omega(P, \mathfrak{g})$, definindo-se

$$
[\omega, \omega](X, Y)=[\omega(X), \omega(Y)]-[\omega(Y), \omega(X)]=2[\omega(X), \omega(Y)]
$$

5. Sejam $\theta \in \Omega^{1}(P, V)$ e $\omega \in \Omega^{1}(P, \mathfrak{g})$. Então, $\omega \wedge \theta \in \Omega^{2}(P, V)$, em que $\omega \wedge \theta(X, Y)=\omega(X) \theta(Y)-\omega(Y) \theta(X)$ Lembrado que $\omega(X) \theta(Y)$ é o produto de uma matriz por um vetor em $\mathbb{R}^{n}$, de modo a estar bem definido.

Sendo $\theta$ a forma tautológica e $\omega$ a 1-forma de conexão, a função

$$
(\theta, \omega)_{p}: T_{p} P \longrightarrow \mathbb{R}^{n} \oplus \mathfrak{g}
$$

é um isomorfismo.

Assim, se

$$
\theta=\theta^{1} e_{1}+\ldots+\theta^{n} e_{n}
$$

onde $e_{1}, \ldots, e_{n}$ é a base canônica de $\mathbb{R}^{n}, \theta^{1}, \ldots, \theta^{n} \in \Omega(\mathcal{S})$ e $\omega=\omega^{1} A_{1}+\ldots+$ $\omega^{m} A_{m}$ onde $A_{1}, \ldots, A_{m}$ é uma base de $\mathfrak{g}$ e $\omega^{1}, \ldots, \omega_{m} \in \Omega(\mathcal{S})$ então formam um correferencial de $\mathcal{S}$. Dessa forma, o produto wedge destas 1-formas são uma base das duas formas em $\Omega(\mathcal{S})$.

Dessa forma, como $d \theta \in \Omega^{2}\left(\mathcal{S}, \mathbb{R}^{n}\right), d \omega \in \Omega^{2}(\mathcal{S}, \mathfrak{g})$, então podemos escre- 
ver

$$
\begin{aligned}
& d \theta=A \theta \wedge \theta+B \omega \wedge \theta+C \omega \wedge \omega \\
& d \omega=a \theta \wedge \theta+b \omega \wedge \theta+c \omega \wedge \omega
\end{aligned}
$$

Proposição 3.4.3. Temos as seguintes igualdades

$$
\begin{aligned}
& d \theta=T(\theta \wedge \theta)+\omega \wedge \theta \\
& d \omega=K(\theta \wedge \theta)-\frac{1}{2}[\omega, \omega]
\end{aligned}
$$

chamadas de equações estruturais.

Demonstração. Vamos determinar os coeficientes $A, B$ e $C$.

Se $X, Y \in T \mathcal{S}$ e $p \in M$, então $X_{p}=X_{p}^{H}+X_{p}^{V}$ e $Y_{p}=Y_{p}^{H}+Y_{p}^{V}$. Desse modo

$$
d \theta(X, Y)=d \theta\left(X^{H}, Y^{H}\right)+d \theta\left(X^{H}, Y^{V}\right)+d \theta\left(X^{V}, Y^{H}\right)+d \theta\left(X^{V}, Y^{V}\right)
$$

e assim, podemos resumir os cálculos em três casos:

1. dois vetores horizontais: seja $H$ o correspondente espaço horizontal correspondente à conexão $\omega$. Então $X_{p}^{H}=\left.\theta\right|_{H_{p}} ^{-1}(u), u \in \mathbb{R}^{n}$ e $Y_{p}^{H}=$ $\left.\theta\right|_{H_{p}} ^{-1}(v), v \in \mathbb{R}^{n}$ e assim

$$
\begin{aligned}
d \theta\left(X_{p}, X_{p}\right) & =d \theta\left(\left.\theta\right|_{H_{p}} ^{-1}(u),\left.\theta\right|_{H_{p}} ^{-1}(v)\right) \\
& =T_{\omega}(u, v) \\
& =T_{p}(\theta(X), \theta(Y))
\end{aligned}
$$

2. um vetor horizontal e outro vertical: sabemos que $R_{g}^{*} \theta=g^{-1} \theta$. Derivando, temos

$$
\begin{aligned}
\mathcal{L}_{\tilde{\alpha}} \theta & =-\alpha \cdot \theta \Rightarrow \\
d i_{\tilde{\alpha} \theta}+i_{\tilde{\alpha} \theta} d \theta & =-\alpha \cdot \theta
\end{aligned}
$$


Portanto

$$
d \theta(\tilde{\alpha}, Y)=-\tilde{\alpha} \cdot \theta(Y)
$$

e assim, se $X$ é vertical e $Y$ é horizontal,

$$
d \theta(X, Y)=-X \cdot \theta(Y)
$$

3. ambos os vetores verticais: estendendo-se $X, Y$ a respectivos campos $\tilde{A}, \tilde{B}$, obtemos

$$
\begin{aligned}
& d \theta(\tilde{A}, \tilde{B})=\underbrace{\mathcal{L}_{\tilde{A}}(\theta(\tilde{B}))}_{=0, \text { pois } \tilde{B} \text { é vertical }}-\underbrace{\mathcal{L}_{\tilde{B}}(\theta(\tilde{A}))}_{=0, \text { pois } \tilde{A} \text { é vertical }}-0 \text {, pois }[\tilde{A}, \tilde{B}] \text { é vertical } \\
& =0
\end{aligned}
$$

de modo que $\mathrm{C}=0$.

Agora vamos determinar $a, b$ e $c$, da mesma maneira que anteriormente:

1. ambos os vetores são horizontais: novamente, se $X_{p}=\left.\theta\right|_{H_{p}} ^{-1}(u), u \in \mathbb{R}^{n}$ e $Y_{p}=\left.\theta\right|_{H_{p}} ^{-1}(v), v \in \mathbb{R}^{n}$, então

$$
\begin{aligned}
d \omega_{p}\left(X_{p}, Y_{p}\right) & =d \omega_{p}\left(\left.\theta\right|_{H_{p}} ^{-1}(u),\left.\theta\right|_{H_{p}} ^{-1}(v)\right) \\
& =K_{p}(\theta(X), \theta(Y)) .
\end{aligned}
$$

(definição de curvatura em fibrados principais).

2. um vetor é horizontal e o outro vertical: sejam $X$ horizontal e $Y=$ $\psi(\beta), \beta \in \mathfrak{g}$ vertical.

Assim:

$$
\begin{aligned}
d \omega(X, Y) & =\mathcal{L}_{X}(\omega(Y))-\mathcal{L}_{Y}(\omega(X))-\omega([X, Y]) \\
& =-\omega([X, Y])
\end{aligned}
$$

$\left(\mathcal{L}_{X}\left(\omega(Y)=0\right.\right.$ pois $\omega(Y)$ é constante e $\mathcal{L}_{Y}(\omega(X))=0$ pois $\left.\omega(X)=0\right)$

X é G-invariante: $d p R_{g}(X)=X, \forall g \Rightarrow[X, Y]=0$. Portanto,

$$
d \omega(X, Y)=0
$$


3. ambos os vetores são verticais: dessa forma $X=\psi(\alpha), Y=\psi(\beta), \alpha, \beta \in$ g. Então

$$
\begin{aligned}
d \omega(X, Y) & =\mathcal{L}_{X}(\omega(Y))-\mathcal{L}_{Y}(\omega(X))-\omega([X, Y]) \\
& =-\omega([X, Y]) \\
& =-\omega([\psi(\alpha), \psi(\alpha)]) \\
& =-\omega(\psi[\alpha, \beta]) \\
& =-[\alpha, \beta]
\end{aligned}
$$

$\left(\mathcal{L}_{X}\left(\omega(Y)=0\right.\right.$ pois $\omega(X)$ é constante e $\mathcal{L}_{Y}(\omega(X))=0$ pois $\omega(X)$ é constante).

Definição 3.4.4. Seja $\omega_{G} \in \Omega(G, \mathfrak{g})$ definida por

$$
\omega_{G}\left(X_{g}\right)=\left(d_{g} L_{g^{-1}}\right)\left(X_{g}\right), X_{g} \in T_{g} G .
$$

$\omega_{G}$ é a chamada forma de Maurer-Cartan.

Definição 3.4.5. Seja $\tau \in \Omega(I)$. O desenvolvimento de $\tau$ em $G$ ao longo de $\boldsymbol{I}=[\boldsymbol{a}, \boldsymbol{b}]$ começando em e é a única função suave $f: I \longrightarrow G$ com $f(a)=$ e tal que $\tau=f^{*}\left(\omega_{G}\right)$.

Definição 3.4.6. Sejam $I=[a, b], N$ uma variedade e $\tau \in \Omega(N, \mathfrak{g})$. Dado uma caminho $\gamma: I \longrightarrow N$, seja $\tilde{\gamma}: I \longrightarrow G$, com $\tilde{\gamma}(a)=e$ o desenvolvimento de $\gamma^{*}(\tau)$, ou seja, $\tilde{\gamma}\left(\omega_{G}\right)=\gamma^{*}(\tau)$. A função $\tilde{\gamma}$ é denominada desenvolvimento de $\tau$ ao longo de $\gamma$ começando em e.

Lema 3.4.1. Seja $G$ um grupo de Lie cuja respectiva álgebra de Lie é g. Se $N$ é uma variedade conexa e $\tau \in \Omega^{1}(N, \mathfrak{g})$ satisfazendo

$$
d \tau+\frac{1}{2}[\tau, \tau]=0
$$

então existe uma função suave $f: N \longrightarrow G$ tal que $\tau=f^{*} \omega_{G}$

Demonstração. (Baseada em [Sha97])

Definamos $f: N \longrightarrow G$, com $f(b)=e$, declarando $f(x)$ como final do desenvolvimento de $\tau$ ao longo de qualquer caminho que vai de $b$ até $x$. 
Vamos obter o valor de $f(x)$. Se $x_{0} \in N$, podemos escolher um caminho de $b$ para $x_{0}$ e outro de $x_{0}$ até $x$. Então, se o desenvolvimento do primeiro caminho começa em $e$ e termina em $g_{0}$, o segundo começa em $e$ e termina em $g$. Então o desenvolvimento da composição dos caminhos começa em $e$ e termina em $g_{0} g$.

A existência loca garante que em torno de cada $x_{0} \in N$, existe um aberto conexo $U$ e $f_{U}: U \longrightarrow G$ satisfazendo $f_{U}^{*}\left(\omega_{G}\right)=\tau$. Após uma translação de $f_{U}$ por algum elemento de $g$, teremos que $f_{U}\left(x_{0}\right)=f(x)$. Dessa forma, o desenvolvimento de $\tau$, começando em $f\left(x_{0}\right)$, ao longo de $\gamma: I \longrightarrow U$ termina em $f_{U}(x)$. Dessa forma,, também termina em $f(x)$, então $f_{U}(x)=f(x)$, para todo $x \in U$. Portanto, $f^{*}\left(\omega_{G}\right)=f_{U}^{*}\left(\omega_{G}\right)$ em $U$.

Teorema 3.4.1. Uma G-estrutura é integrável, se e somente, $M$ admite, localmente, conexões flat livre de torção.

Demonstração. Podemos tratar localmente o problema, de modo assumir a variedade $M$ como sendo $\mathbb{R}^{n}$, e analisar em torno de 0 . Se uma $G$-estrutura é integrável, todo referencial local é isomorfo ao referencial canônico, de modo a ser flat a conexão e livre de torção.

Agora vamos provar a volta. Se $M$ admite uma conexão flat livre de torção, então as equações estruturais tornam-se

$$
\begin{gathered}
d \theta=\omega \wedge \theta, \\
d \omega=-\frac{1}{2}[\omega, \omega] .
\end{gathered}
$$

Vamos considerar uma nova álgebra de Lie

$$
\overline{\mathfrak{g}}=\mathfrak{g} \oplus V
$$

chamado de produto semi-direto de $\mathfrak{g}$ com sua representação em $V$, cujo colchete é definido como

$$
[(A, u),(B, v)]=([A, B], A u-B v) .
$$

Essa álgebra de Lie advém do grupo $\bar{G}=G \times V$ tal que $(g, u)(h, v)=$ $\left(g h, h^{-1}(u) v\right)$

Podemos, juntando $\omega$ e $\theta$, formando a 1-forma $\Theta=(\omega, \theta) \in \Omega(\mathcal{S}, \overline{\mathfrak{g}})$. 
Dessa forma, as equações estruturais, são reescritas como

$$
d \Theta+\frac{1}{2}[\Theta, \Theta]=0
$$

Pelo lema, existe uma função $F: \mathcal{S} \longrightarrow \bar{G}$ de modo que $F^{*}\left(\omega_{\bar{G}}\right)=\Theta$. Como a questão é local, podemos adotar $V=\mathbb{R}^{n}$ e vamos considerar o seguinte diagrama:

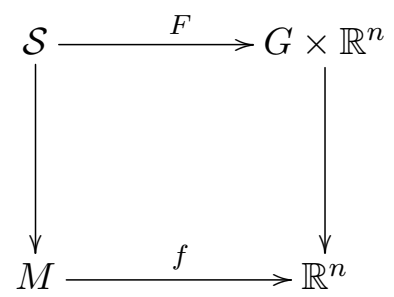

que obtemos ao passar ao quociente módulo $G$. F é o levantamento, ao menos localmente, de um difeomorfismo $f: U \subseteq M \longrightarrow \mathbb{R}^{n}$, onde $U$ é o domínio de uma carta local. A correspondente carta será uma carta adaptada, provando que $M$ é localmente integrável. 


\section{Capítulo 4}

\section{Integração de G-estruturas}

Este capítulo baseia-se em [Gui65] e nas notas [dA17] do colega Pedro Mendes de Araújo.

Neste capítulo discutiremos com mais atenção o problema da integrabilidade de G-estruturas através da cohomologia de Spencer, cujos grupos de cohomologia representam os obstáculos para se determinar a integrabilidade.

\subsection{Prolongamentos e Cohomologia de Spencer}

Sejam $V$ um espaço vetorial real de dimensão $n, G \subseteq G L_{n}(V)$ um grupo de Lie e $\mathfrak{g} \subseteq g l_{n}(V)=\operatorname{End}(V)$ sua respectiva álgebra de Lie.

Definição 4.1.1. O k-ésimo prolongamento de $\mathfrak{g}$, denotado por $\mathfrak{g}^{(k)}$ é definido indutivamente da seguinte maneira: $\mathfrak{g}^{(1)}$ é o conjunto das transformações lineares $T: V \longrightarrow \mathfrak{g}$ tal que $T(u)(v)=T(v)(u)$, para $u, v \in V$ (esta definição coincide com a dada no capítulo anterior). Da mesma forma, $\mathfrak{g}^{(k)}$ é o conjunto das transformações lineares $T: V \longrightarrow \mathfrak{g}^{(k-1)}$ tal que $T(u)(v)=T(v)(u), u, v \in V$.

Assim, $g^{(k)} \subseteq \operatorname{Hom}\left(V, \mathfrak{g}^{(k-1)}\right)$ pode ser descrito diretamente por

$$
\mathfrak{g}^{(k)}=\left(\mathfrak{g} \otimes S^{k}\left(V^{*}\right)\right) \cap\left(V \otimes S^{k+1}\left(V^{*}\right)\right)
$$

Temos $\mathfrak{g}^{(0)}=\mathfrak{g}$ e vamos definir $\mathfrak{g}^{(-1)}=V$.

Considerando $v \in V$, este pode ser visto como um elemento de $\left(V^{*}\right)^{*}$. Podemos estender $v$ a uma derivação $D_{v}$ em $S\left(V^{*}\right)$ : se $\lambda_{1}, \ldots, \lambda_{n}$ é uma base 
de $V^{*}$, um elemento de $S\left(V^{*}\right)$ pode ser escrito como

$$
\sum_{i_{1}, \ldots, i_{k}=1}^{n} a_{i_{1} \ldots i_{k}} \lambda_{i_{1}} \otimes \ldots \otimes \lambda_{i_{k}}
$$

Definindo-se

$$
D_{v}\left(\lambda_{i}\right)=\lambda_{i}(v)
$$

temos

$$
D_{v}\left(\lambda_{i} \otimes \lambda_{j}\right)=\lambda_{i}(v) \lambda_{j}+\lambda_{j}(v) \lambda_{i}
$$

de modo a termos a aplicação

$$
\begin{gathered}
D_{v}: S\left(V^{*}\right) \longrightarrow S\left(V^{*}\right) \\
D_{v}(p q)=D_{v}(p) q+p D_{v}(q)
\end{gathered}
$$

Assim,

$$
D_{v}\left(\sum_{i_{1}, \ldots, i_{k}=1}^{n} a_{i_{1} \ldots i_{k}} \lambda_{i_{1}} \otimes \ldots \otimes \lambda_{i_{k}}\right)=\sum_{1 \leq l \leq k} \sum_{i_{1}, \ldots, i_{k}=1}^{n} a_{i_{1} \ldots i_{k}} \lambda_{i_{l}}(v) \lambda_{i_{1}} \ldots \lambda_{i_{k}}
$$

Sejam $u \otimes s \in V \otimes S^{r+1}\left(V^{*}\right)$ e $v \otimes t \in V \otimes S^{s+1}\left(V^{*}\right)$. Então, definido um colchete entre esses elementos por

$$
[u \otimes s, v \otimes t]=v \otimes D_{u} t \circ s-u \otimes D_{v} s \circ t \in V \otimes S^{r+s+1}\left(V^{*}\right)
$$

pois $s \in S^{r+1}\left(V^{*}\right), t \in S^{s+1}\left(V^{*}\right)$, de modo que

$D_{v} t \in S^{s+1}\left(V^{*}\right) \Rightarrow D_{v} t \circ s \in S^{r+s+1}\left(V^{*}\right)$ e $D_{u} s \in S^{r+1}\left(V^{*}\right) \Rightarrow D_{u} s \circ t \in S^{r+s+1}\left(V^{*}\right)$

Como $\mathfrak{g}^{(r)} \subseteq V \otimes S^{r+1}\left(V^{*}\right)$ e $\mathfrak{g}^{(s)} \subseteq V \otimes S^{s+1}\left(V^{*}\right)$, o colchete acima induz uma aplicação bilinear $\mathfrak{g}^{(r)} \otimes \mathfrak{g}^{(s)} \longrightarrow \mathfrak{g}^{(r+s)} \subseteq V \otimes S^{r+s+1}\left(V^{*}\right)$ : se $\alpha \in \mathfrak{g}^{(r)}$ e $\beta \in \mathfrak{g}^{(s)}$ então $[\alpha, \beta] \in \mathfrak{g}^{(r+s)}$.

O colchete apresentado anteriormente faz de $\bigoplus_{k=-1}^{+\infty} \mathfrak{g}^{(k)}$ uma álgebra de Lie.

Definição 4.1.2. Sejam $p, q \geq 0$ números naturais. Então, definimos o 
seguinte complexo duplo

$$
C^{p, q}=\mathfrak{g}^{(p-1)} \otimes \Lambda^{q}\left(V^{*}\right)
$$

Se $v \in V$ e $\alpha \in \mathfrak{g}^{(k)}$, temos

$$
[v, \alpha]=\alpha(v) \in \mathfrak{g}^{(k-1)}
$$

Dado $s \in C^{p, q}$, definamos

$$
\delta(s)(u, v)=D_{u}(s \circ v)-D_{v}(s \circ u), \delta(s) \in C^{p-1, q+1}
$$

uma derivação de bigrau $(-1,1)$. Se calcularmos $\delta^{2}$ veremos que $\delta^{2}=0$, ou seja , é uma derivação

Definição 4.1.3. Os grupos de cohomologia de Spencer são definidos como

$$
H^{p, q}(\mathfrak{g})=\frac{\operatorname{ker} \delta^{p, q}}{\operatorname{Im}\left(\delta^{p+1, q-1}\right)}
$$

Os grupos de cohomologia de Spencer contém informações sobre a obstrução da integrabilidade de uma G-estrutura, através de tensores com valores nestes grupos.

\subsection{Contato de k-ésima ordem e jatos.}

Sejam $M$ e $N$ duas variedades diferenciáveis tais que $f: M \longrightarrow N$ é um difeomorfismo, de modo que $f$ seja uma equivalência de $G$-estruturas em $M$ e $N$, respectivamente.

Definição 4.2.1. Sejam $M$ uma variedade suave e $M_{1}$ e $M_{2}$ duas subvariedades de mesma dimensão $n$ que possuem um ponto comum $x$. Dizemos que $M_{1}$ e $M_{2}$ têm contato de $\boldsymbol{k}$-ésima ordem em $p$ se é possivel encontrar um sistema de coordenadas $\left(y_{1}, \ldots, y_{n}\right)$ de $M$ em torno de $x$, de modo que para $M_{1}, y_{j}=0, m+1 \leq j \leq n$ e para $M_{2} y_{j}=f_{j}\left(y_{1}, \ldots, y_{n}\right), m+1 \leq j \leq n$, com todas as derivadas de ordem menor ou iguais a $k$ de $f_{j}$ anulando-se.

Definição 4.2.2. Sejam $M_{1}$ e $M_{2}$ duas variedades suaves e $f, f: U \longrightarrow V$ funções suaves de uma vizinhança $U$ de $x \in M_{1}$ em $V$ uma vizinhança de $y=f(x)=f^{\prime}(x) \in M_{2}$. Estas funções concordam até ordem $k$ em $x$ 
se têm a mesma expansão de Taylor até a k-ésima ordem $(k \in[0, \infty])$ em alguma carta coordenada em torno de $x$ e y. O $k$-jato em $x$ é a classe de equivalência de todas as funções que concordam até a ordem $k$ em $x$ e denota-se por $j_{x}^{k}(f)$. O ponto $x$ é chamado de origem do jato e $f(x)$ o alvo do jato.

Definição 4.2.3. Sejam $\mathcal{S}_{1}$ e $\mathcal{S}_{2} G$-estruturas em $M_{1}$ e $M_{2}$, respectivamente e $f: M_{1} \longrightarrow M_{2}$ é um difeomorfismo e $x \in M_{1} \cap M_{2}$. A função $f$ preserva $G$-estruturas até $k$-ésima ordem se se algum referencial de $\mathcal{S}_{x}^{1}$ é levado por $d f_{x}$ em outro referencial de $\mathcal{S}_{f(x)}^{2}$ e $f\left(\mathcal{S}^{1}\right)$ e $\mathcal{S}^{2}$ têm contato de k-ésima ordem.

Vamos denotar $J_{x}^{k}(M, N)$ o conjunto dos jatos de ordem $k$ das funções $f$ : $M \longrightarrow N$ com origem em $x$. Se $l<k$, existe uma projeção $\pi_{l}^{k}: J_{x}^{k}(M, N) \longrightarrow$ $J_{x}^{l}(M, N), j_{x}^{k}(f) \mapsto j_{x}^{l}(f)$

Observação 11. Se tomarmos uma outra função g que tem o mesmo $(k+1)$ jato de $f$ em $x$, as condições da definição são igualmente satisfeitas. Assim, a definição anterior não depende de $f$ em si mas do $(k+1)$-jato de $f$ em $x$. Portanto, podemos dizer que um $j_{x}^{k+1} f$ preserva a estrutura até ordem $k$.

\subsection{Fibrados referenciais e forma tautológica}

Definição 4.3.1. O k-ésimo fibrado referencial é o conjunto

$$
F^{k}(M)=\left\{j_{0}^{k+1} f \mid f \text { é um difemorfismo local de } M \text { em } V\right\}
$$

Quando $k=0$, obtemos o fibrado referencial do capítulo anterior: $\mathrm{Fr}^{0}(M)=$ $\left\{\phi: V \longrightarrow T_{x} M: \phi\right.$ é isomorfismo linear $\}$.

$$
\begin{gathered}
G L_{n}^{k}=\left\{j_{0}^{k+1} g \mid g: V \longrightarrow V \text { é difeomorfismo que leva } 0 \text { em } 0\right\} \\
E^{k}=\left\{j_{0}^{k+1} f \mid f: V \rightarrow M \text { preserva estrutura até } k \text {-ésima ordem em } 0\right\} . \\
E_{0}^{k}=\left\{j_{0}^{k+1} f \in F r^{k}(M) \mid f: V \rightarrow V \text { preserva estrutura até k-ésima ordem em } 0\right\} . \\
G^{k}=\left\{j_{0}^{k+1} g \in G L_{n}^{k} \mid g \text { preserva estrutura standart até } k \text {-ésima ordem em } 0\right\}
\end{gathered}
$$

Observação 12. $E=E^{1}$ é a G-estrutura cuja integrabilidade queremos investigar e $E_{0}$ a G-estrutura padrão e $E^{k} \rightarrow M$ é um $G^{k}$-fibrado principal. 
Pela decomposição de um jato em suas derivadas, obtemos um isomorfismo

$$
J_{0}^{k} T V \simeq V \oplus \mathfrak{g l} \oplus \mathfrak{g l}^{(1)} \oplus \mathfrak{g l}^{(2)} \oplus \ldots \oplus \mathfrak{g l}^{(k-1)}
$$

Vamos definir o seguinte colchete

$$
\begin{gathered}
{[,]: J_{0}^{k} T V \times J_{0}^{k} T V \longrightarrow J_{0}^{k-1} T V} \\
{\left[j_{0}^{k} X, j_{0}^{k} Y\right]=j_{0}^{k-1}[X, Y]}
\end{gathered}
$$

Este colchete está bem definido pois o resultado depende dos $k$ - jatos em 0 de $X$ e $Y$, que não dependem de $X$ e $Y$.

Consideremos a aplicação

$$
\begin{gathered}
\Phi: J_{0}^{k+1} T V \longrightarrow T_{j_{0}^{k+1} f} F r^{k}(M) \\
\Phi\left(j_{0}^{k+1} X\right)=\left.\frac{d}{d t}\right|_{t=0} j_{0}^{k+1}(f \circ \exp (t X))
\end{gathered}
$$

Definição 4.3.2. A forma tautológica de ordem $\boldsymbol{k} \theta^{k} \in \Omega^{1}\left(F r^{k}(M), J_{0}^{k} T V\right)$, é definida como

$$
\theta_{j_{0}^{k+1} f}^{k}=\Phi^{-1} \circ d_{j_{0}^{k+1} f} \pi_{k-1}^{k}
$$

onde $\pi_{k-1}^{k}: F r^{k}(M) \rightarrow F r^{k-1}(M)$ é a projeção.

Seja $\rho: G L^{k} \longrightarrow A u t\left(J_{0}^{k} T V\right)$,

$$
\rho\left(j_{0}^{k+1} h\right)\left(j_{0}^{k} X\right)=\left.\frac{d}{d t}\right|_{t=0} j_{0}^{k}\left(h \circ \exp (t X) \circ h^{-1}\right)
$$

uma representação.

\section{Lema 4.3.1.}

$$
R_{g}^{*} \theta^{k}=\rho\left(j_{0}^{k+1} h\right) \theta^{k}
$$


Demonstração. Seja $\tilde{X}=\left.\frac{d}{d t}\right|_{t=0} j_{0}^{k+1}(f \circ \exp (t X))$ em $j_{0}^{k+1} f \in F r^{k}$. Então

$$
\begin{aligned}
R_{g}^{*} \theta^{k}(\tilde{X}) & =\theta_{j_{0}^{k+1}(f \circ h)}^{k}\left(d_{j_{0}^{k+1} f} R_{g}(\tilde{X})\right) \\
& =\theta_{j_{0}^{k+1}(f \circ h)}^{k}\left(\left.\frac{d}{d t}\right|_{t=0} j_{0}^{k+1}\left(h \circ \exp (t X) \circ h^{-1}\right)\right) \\
& =\left.\frac{d}{d t}\right|_{t=0} j_{0}^{k}\left(h \circ \exp (t X) \circ h^{-1}\right) \\
& =\rho\left(j_{0}^{k+1} h\right) \theta^{k}
\end{aligned}
$$

Proposição 4.3.1. Seja $\tau: J_{0}^{k} T V \longrightarrow J_{0}^{k-1} T V$ projeção. Então

$$
d \tau \theta^{k}+\frac{1}{2}\left[\theta^{k}, \theta^{k}\right]=0
$$

Demonstração. Sejam

$$
\bar{X}=\left.\frac{d}{d t}\right|_{t=0} j_{0}^{k}(f \circ \exp (t X)), \bar{Y}=\left.\frac{d}{d t}\right|_{t=0} j_{0}^{k}(f \circ \exp (t Y))
$$

vetores $\operatorname{em} T_{j_{0}^{k+1} f} F r^{k}(M), \operatorname{com} X, Y \in \Gamma(T V)$.

Assim,

$$
\begin{aligned}
d \theta^{k}(\bar{X}, \bar{Y}) & =\underbrace{\bar{X}\left(\theta^{k}(\bar{Y})\right)}_{\left.=0\left(\theta^{k}(\bar{Y})\right) c t e .\right)}-\underbrace{\bar{Y}\left(\theta^{k}(\bar{Y})\right)}_{\left.=0\left(\theta^{k}(\bar{X})\right) c t e .\right)}-\theta^{k}([\bar{X}, \bar{Y}]) \\
& =\theta^{k}([\bar{X}, \bar{Y}])
\end{aligned}
$$

Mas

$$
\begin{aligned}
\theta^{k}([\bar{X}, \bar{Y}]) & =\left[\theta^{k}(\bar{X}), \theta^{k}(\bar{Y})\right] \\
& =\frac{1}{2}\left[\theta^{k} \theta^{k}\right](\bar{X}, \bar{Y})
\end{aligned}
$$

Temos

$$
\left.\tau \theta^{[} \bar{X}, \bar{Y}\right]=\left[\theta^{k}(\bar{X}), \theta^{k}(\bar{Y})\right]
$$




\subsection{Funções estruturais}

Podemos escrever a forma tautológica como

$$
\theta^{k}=\omega \oplus \Omega^{0} \oplus \ldots \oplus \Omega^{k-1}
$$

em que $\omega, \Omega^{0}, \ldots, \Omega^{k-1}$ são as partes homogêneas de $\theta^{k}: \omega \in \Omega^{1}\left(F r^{k}(M), V\right), \Omega^{i} \in$ $\left(\operatorname{Fr}^{k}(M), \mathfrak{g}^{(i)}\right)$

Sejam $E$ uma $G$-estrutura e $j_{0}^{k+1} f \in E^{k}$. Seja $H$ um espaço horizontal contido em $T_{\phi} E^{k}$, no qual as formas $\left.\Omega^{0}\right|_{H}=\ldots=\left.\Omega^{k-1}\right|_{H}=0$. Em geral, tal subespaço não é único.

Seja $\pi^{k}: E^{k} \rightarrow M$ a projeção e $\zeta:=\left(\left.d_{\phi} \pi^{k}\right|_{H}\right)^{-1}$, onde $\phi=j_{0}^{k+1} f \in E^{k}$. Então, definamos a função

$$
\begin{gathered}
\Pi_{H}: \Lambda^{2} V \longrightarrow V \oplus \mathfrak{g} \oplus \mathfrak{g}^{(1)} \oplus \mathfrak{g}^{(2)} \oplus \ldots \oplus \mathfrak{g}^{(k-1)} \\
\Pi_{H}(u, v)=d \theta^{k}\left(\zeta\left(f_{*} u\right), \zeta\left(f_{*} v\right)\right)
\end{gathered}
$$

Podemos escrever $\Pi_{H}$ como uma soma

$$
\Pi_{H}=\Pi_{H}^{(-1)} \oplus \Pi_{H}^{(0)} \oplus \ldots \oplus \Pi_{H}^{(k-1)}
$$

ou seja, $\Pi_{H}^{(j)}, j=-1, \ldots, k-1$ são suas partes homogêneas.

Proposição 4.4.1.

$$
\Pi_{H}^{(i)}=0, \text { para } i<k-1 .
$$

Demonstração. Seja $d \Omega^{i} \circ H$ a parte horizontal de $d \Omega^{i}$.

Provemos que vale a identidade

$$
d \Omega^{i} \circ H=d \Omega^{i}+\frac{1}{2}\left(\left[\omega, \Omega^{i+1}\right]+\left[\Omega^{0}, \Omega^{i}\right], \ldots,\left[\Omega^{i+1}, \omega\right]\right) .
$$

Cada lado desta igualdade é horizontal, de modo que, se aplicarmo-os a vetores verticais, ambos anular-se-ão.

Mas as partes homogêneas anulam-se em vetores horizontais, de modo que ao aplicar o lado direito em $\mathrm{m}$ vetores horizontais $u, v$,obtemos $d \Omega^{i}(u, v)$. Mas obtemos a mesma coisa do lado direito, de modo a valer a identidade.

O lado direto é identicamente nulo, para $i, k-1$; basta usar a proposição 4.3 .1 
Ao diferenciar ambos os lado direito (1.1), quando $i=k-2$, obtemos

$$
\left[d \Omega^{k-1} \circ H, \omega\right]=0
$$

Ou seja, $\delta \Pi_{H}^{(k-1)}=0$, de modo que a induzir uma classe de cohomologia $\left[\Pi_{H}^{(k-1)}\right] \in H^{k, 2}(\mathfrak{g})$.

Proposição 4.4.2. A classe $\left[\Pi_{H}^{(k-1)}\right]$ não depende da escolha do espaço horizontal $H$.

Demonstração. Sejam $H$ e $H^{\prime}$ duas escolhas de espaço horizontal. Definamos $s_{H, H^{\prime}}: V \longrightarrow \mathfrak{g}^{(k)}$

$$
a\left(s_{H, H^{\prime}}(u)\right)_{\phi}=\zeta\left(f_{*} u\right)-\zeta^{\prime}\left(f_{u}\right)
$$

onde $a: V \rightarrow V \oplus \mathfrak{g} \oplus \mathfrak{g}^{(1)} \oplus \mathfrak{g}^{(2)} \oplus \ldots \oplus \mathfrak{g}^{(k-1)}$ é o função de geradores infinitesimais e $\zeta^{\prime}$ o levantamento horizontal em relação a $H$. Como $H$ e $H^{\prime}$ são aniquilados por $\Omega^{0}, \ldots, \Omega^{k-1}$, então $s_{H H^{\prime}}$ assume, de fato, valores em $\mathfrak{g}^{(k)}$.

Vamos mostrar que $\Pi_{H^{\prime}}^{(k-1)}-\Pi_{H}^{(k-1)}=\delta s_{H H^{\prime}}$.

$$
\begin{aligned}
\Pi_{H^{\prime}}^{(k-1)}(u, v)-\Pi_{H}^{(k-1)}(u, v) & =d \theta^{k+1}\left(\zeta^{\prime}\left(f_{*} u\right), \zeta^{\prime}\left(f_{*} v\right)\right)-d \theta^{k+1}\left(\zeta\left(f_{*} u\right), \zeta\left(f_{*} v\right)\right) \\
& =d \theta^{k+1}\left(a\left(s_{H, H^{\prime}}(v)\right), \zeta^{\prime}\left(f_{*} u\right)\right)-d \theta^{k+1}\left(a\left(s_{H, H^{\prime}}(v)\right) \zeta^{\prime}\left(f_{*} u\right)\right) \\
& \left.\left.=\left(a\left(s_{H H^{\prime}}(v)\right)\right\lrcorner d \theta^{k+1}\right)\left(\zeta\left(f_{*} u\right)\right)-\left(a\left(s_{H H^{\prime}}(u)\right)\right\lrcorner d \theta^{k+1}\right)\left(\zeta\left(f_{*} v\right)\right)
\end{aligned}
$$

Ao diferenciarmos a condição $R_{g}^{*} \theta^{k+1}=g^{-1} \theta^{k+1}$, obtemos

$$
\begin{aligned}
\left.\left(a\left(s_{H H^{\prime}}(v)\right)\right\lrcorner d \theta^{k+1}\right)\left(\zeta\left(f_{*} u\right)\right) & =\left(\mathcal{L}_{a\left(s_{H H^{\prime}}(v)\right)} \theta^{k+1}\right)\left(\zeta\left(f_{*} u\right)\right) \\
& =-s_{H H^{\prime}}(v) u .
\end{aligned}
$$

Desse modo,

$$
\Pi_{H^{\prime}}^{(k-1)}(u, v)-\Pi_{H}^{(k-1)}(u, v)=s_{H H^{\prime}}(u) v-s_{H H^{\prime}}(v) u
$$

Seja $c^{k}: E^{k} \longrightarrow H^{k, 2}(\mathfrak{g})$ a função que associa a cada ponto de $E^{k}$ a uma classe de cohomologia de $H^{k, 2}(\mathfrak{g})$. Essa função é chamada de k-ésimo 
tensor de estrutura de $\mathbf{E}$.

\subsection{G-estruturas uniformemente $k$-integráveis}

Definição 4.5.1. Uma G-estrutura é uniformemente k-integrável se, em cada ponto $x \in M$, existe um $(k+1)$-jato de origem em $x$ e alvo em $0 \in V$ que preserva estrutura até $k$-ésima ordem.

A integrabilidade G-estruturas depende de $H^{i, j}$, que anula-se para $i$ suficientemente grande de modo que a integração de uma G-estrutura é determinada por um número finito de passos.

Teorema 4.5.1. Suponhamos que $E \longrightarrow M$ é uniformemente $k$-integrável e seja $x \in M$. Se o k-ésimo tensor de estrutura anula-se em $x$, existe um $(k+2)$-jato com origem em $0 \in V$ e alvo em $x$ que preserva estrutura até $(k+1)$-ésima ordem.

Demonstração. Seja $\phi \in E^{k}$ na fibra sobre $x$. O fato de $c^{k}$ anular-se implica na existência de um espaço horizontal $H$ para o qual $d \Omega^{k-1} \circ H=0$, ou seja,

$$
d \Omega^{k-1}+\frac{1}{2}\left(\left[\omega, \Omega^{k}\right]+\left[\Omega^{0}, \Omega^{k-1}\right]+\ldots+\left[\Omega^{k}, \omega\right]\right)=0
$$

e se $X \in \mathfrak{g}^{k}$ onde o correspondente vetor $\tilde{X} \in T_{\phi} E^{k}, \Omega^{k} \tilde{X}$ é a componente de $X$ em $\mathfrak{g}^{(k)}$.

A 1-forma $\omega \oplus \Omega^{0} \oplus \ldots \oplus \Omega^{k-1}$ estabelece uma função $\gamma: T_{\phi} E^{k} \longrightarrow T_{i_{k}} E_{0}^{k}$. Podemos estendê-la a uma função $\bar{\gamma}: T_{\phi} F^{k} \longrightarrow T_{i_{k}} F_{0}^{k}$. Vamos provar que esta função é induzida por algum $(k+2)$-jato de alguma função $M \longrightarrow V$, com fonte $x$ e alvo 0 .

Considerando $\left(f_{1}, \ldots, f_{k}\right)$ as funções que definem $\bar{\gamma}$, e a proposição 4.4.1, cada $(k+2)$-jato induz 1-jatos satisfazendo equações análogas a 4.3 e quantidade desses $(k+2)$-jatos que estendem $(k+1)$-jatos é igual ao número de soluções de tais equações.

Sendo $j_{x}^{k+2} f$ o jato que origina $\bar{\gamma}$, a função $f$ preserva até $k+1$-ésima ordem pois é uma função $T_{\phi} E^{k} \longrightarrow T_{i_{k}} E_{0}^{k}$. 


\subsection{G-estruturas de tipo finita}

Definição 4.6.1. Um subgrupo $G \subseteq G L_{n}$ é de tipo finito se existe $k \geq 0$ tal que $\mathfrak{g}^{(k)}=0$. Se $k$ é tal que $\mathfrak{g}^{(k-1)} \neq 0$ e $\mathfrak{g}^{(k)}=0$ então a $G$ é dito de tipo finito do tipo $k$.

Teorema 4.6.1. Seja $G$ um grupo do tipo $k$. Então toda $G$-estrutura $(k+1)$ uniformemente integrável será integrável.

Demonstração. Como $H^{k+1,2}(\mathfrak{g})=0$, a função $c^{k+1}$ será nula. Desse modo, pelo teorema 4.5.1, a $G$-estrutura será $(k+2)$-integrável.

Além disso, já que $\mathfrak{g}^{(k)}=\mathfrak{g}^{(k+1)}=0$, então $E^{k+1}$ e $E^{k+2}$ projetam-se isomorficamente sobre $E^{k}$. Dessa maneira, também são idênticas as formas $\theta^{k+1}$ e $\theta^{k+2}$.

Como provamos, vale a relação

$$
d \tau \theta^{k+1}+\frac{1}{2}\left[\theta^{k+1}, \theta^{k+1}\right]=0
$$

e, pelo Teorema A.5.1, existe um difeomorfismo $f: U \subseteq E^{k} \longrightarrow E_{0}^{k}$, com $f^{*} \theta_{0}^{k}=\theta^{k}$, onde $E_{0}^{k}=V \rtimes G^{k}$ é a G-estrutura standart de $V=\mathbb{R}^{n}$, que é identificada com o grupo dado pelo produto semi-direto e $\theta_{0}^{k}$ é a froma de Maurer-Cartan deste grupo.

Assim, f induz uma aplicação $\gamma: W \subseteq M \longrightarrow V$ com levantamento $\gamma^{\prime}: E \longrightarrow E_{0}$. 


\section{Apêndice A}

\section{Apêndice}

\section{A.1 Variedades: quocientes}

Definição A.1.1. Se $M$ é uma variedade e $M \times G \longrightarrow M$ é uma ação á direita de um grupo de Lie, então a ação é dita

1. livre se $x g=g$ então $x=e$

2. própria se a aplicação $M \times G \longrightarrow M \times M,(x, g) \mapsto(x, x g)$ é própria: a imagem inversa de todo compacto contido no contra domínio por essa aplicação é uma compacto contido no domínio.

Teorema A.1.1. Seja $M$ uma variedade e $\sim$ uma relação de equivalência M. São equivalentes:

1. Existe uma estrutura diferenciável em $M / \sim$ tal que $\pi: M \longrightarrow M / \sim$ é uma submersão.

2. O gráfico de $R=\{(x, y) \in M \times M: p \sim q\}$ é uma subvariedade própria de $M \times M$ e a projeção $p_{1}: M \times M \longrightarrow M$ restrita a $R$ é uma submersão.

Demonstração. Ver [Fer03], página 43.

Teorema A.1.2. Sejam $G$ um grupo de Lie e $M$ uma variedade diferenciável e a: $M \times G \longrightarrow M$ uma ação á direita diferenciável, livre e própria. Então $M / G$ possui estrutura de variedade diferenciável tal que a projeção $\pi: M \longrightarrow M / G$ é uma submersão. Em particular, $\operatorname{dim} M / G=$ $\operatorname{dim} M-\operatorname{dim} G$. 
Demonstração. (Retirado de [Fer03])

Relembrando que a relação de equivalência é dada por:

$$
x \sim y, x, y \in M \Longleftrightarrow \exists g \in G \text { tal que } x g=y \text {. }
$$

Vamos aplicar o teorema anterior a esta relação, verificando que

$$
\Gamma=\{(x, x g): x \in M, g \in G\} \subseteq M \times M
$$

é uma subvariedade própria e a projeção $\left.p r_{1}\right|_{\Gamma}: \Gamma \longrightarrow M$ é uma submersão.

Vamos considerara a função

$$
\rho: M \times G \longrightarrow M \times M, \rho(x g)=(x, x g) .
$$

A imagem de $\rho$ é exatamente $\Gamma$. A ação é livre, de modo que $\rho$ é injetora. Seja sua diferencial em $(x, g)$ a função

$$
d_{(x, g)} \rho: T_{x} M \times T_{g} G \longrightarrow T_{x} M \times T_{x g} M
$$

dada por

$$
d_{(x, g)} \rho(u, v)=\left(u, u d_{x} a+v d_{g} a\right) .
$$

A ação $a_{x}$ é uma imersão, portanto injetora. Assim, $\rho$ é uma imersão injetora cuja imagem é $\Gamma$. Por hipótese, $\rho$ é própria, de modo que $\Gamma$ é uma subvariedade própria de $M \times M$.

A composição $p r_{1} \circ \rho: M \times G \longrightarrow M$ é definida por $\rho(p, g)=p$ é uma submersão. Logo, $\left.p r_{1}\right|_{\Gamma}$ é uma submersão.

\section{A.2 Cálculos de Cartan}

(Baseado em [Lee97] e [Tu10])

\section{A.2.1 Derivada de Lie de um campo vetorial}

Sejam $M$ uma variedade diferenciável, $p \in M, X, Y \in \mathfrak{X}(M)$ e $\varphi$ : ]$\varepsilon, \varepsilon[\times U \longrightarrow M$ um fluxo local de $X$ em $U$ uma vizinhança de $p$.

Definição A.2.1. A derivada de Lie de $Y$ com respeito a $X$ em $P$ é dado 
por

$$
\left(\mathcal{L}_{X} Y\right)_{p}=\lim _{t \rightarrow 0} \frac{\varphi_{t}^{*}\left(Y_{\varphi_{y}(p)}\right)-Y_{p}}{t}=\lim _{t \rightarrow 0} \frac{\left(\varphi_{t}^{*} Y\right)_{p}-Y_{p}}{t}=\left.\frac{d}{d t}\right|_{t=0}\left(\varphi_{t}^{*} Y\right)_{p}
$$

Teorema A.2.1. Se $X$ e $Y$ são campos suaves em $M$, então $\mathcal{L}_{X} Y=[X, Y]$ Demonstração. Ver [Tu10], página 225.

\section{A.2.2 Contração ou produto interior}

Seja $V$ um espaço vetorial real de dimensão finita $n$. Considere $v \in V$ e $\omega \in \Lambda^{k}(V)$.

Definição A.2.2. Chamamos de produto interior ou contração de $\omega$ por $v$ à função linear $i_{v}: \Lambda^{k}\left(V^{*}\right) \longrightarrow \Lambda^{k-1}\left(V^{*}\right)$ tal que

$$
i_{v} \omega\left(w_{1}, \ldots, w_{k-1}\right)=\omega\left(v, w_{1}, \ldots, w_{k-1}\right)
$$

Denota-se $i_{v} \omega$ também por $\left.v\right\lrcorner \omega$

Proposição A.2.1. Sejam $V$ um espaço vetorial real de dimensão finita $n$ $e v \in V$. Então:

1. $i_{v} \circ i_{v}=0$

2. se $\omega \in \Lambda^{k}\left(V^{*}\right)$ e $\tau \in \Lambda^{l}\left(V^{*}\right)$, então

$$
i_{v}(\omega \wedge \tau)=i_{v} \omega \wedge \tau+(-1)^{k} \omega\left(i_{v} \tau\right)
$$

Demonstração. 1. Se $\omega \in \Lambda^{k}\left(V^{*}\right)$, então

$$
\begin{gathered}
i_{v}\left(i_{v}(\omega)\right)=i_{v}\left(\omega\left(v, w_{1}, \ldots, w_{k-1}\right)\right) \\
\omega\left(v, v, w_{1}, \ldots, w_{k-1}\right) \\
=0
\end{gathered}
$$

2. Basta considerar o caso em que as formas decomponíveis já que todo tensor de posto positivo é combinação linear de outro decomponíveis. É suficiente provar 


$$
i_{v}\left(\omega \wedge \ldots \wedge \omega^{k}\right)=\sum_{i=1}^{i-1}(-1)^{i-1} \omega^{i}(v) \omega \wedge \ldots \wedge \hat{\omega}^{i} \ldots \wedge \omega^{k}
$$

Adotando $v_{1}=v$, temos:

$$
\left(\omega \wedge \ldots \wedge \omega^{k}\right)\left(v_{1}, \ldots, v_{k}\right)=\operatorname{det}\left[\omega^{i}\left(v_{j}\right)\right]
$$

Por outro lado

$$
\sum_{i=1}^{i-1}(-1)^{i-1} \omega^{i}\left(v_{1}\right)\left(\omega \wedge \ldots \wedge \hat{\omega^{i}} \ldots \wedge \omega^{k}\right)\left(v_{2}, \ldots, v_{k}\right)=\sum_{i=1}^{i-1}(-1)^{i-1} \omega^{i}\left(v_{1}\right) \operatorname{det}\left[M_{i j}\right]
$$

em que $\left[M_{i j}\right]$ é a matriz obtida de $\left[\omega^{i}\left(v_{j}\right)\right]$ ao eliminar-se a linha $i$ e a colune $j$.

\section{A.2.3 Derivadas de Lie em formas diferenciais}

Definição A.2.3. Seja $X \in \mathfrak{X}(M)$ suave, $\omega \in \Omega^{k}(M)$. A derivada $\mathcal{L}_{X} \omega$ em $x \in M$ é

$$
\left(\mathcal{L}_{X} \omega\right)_{x}=\lim _{t \rightarrow 0} \frac{\varphi_{t}^{*}\left(\omega_{\varphi_{t}(x)}\right)-\omega_{x}}{t}=\lim _{t \rightarrow 0} \frac{\left(\varphi_{t}^{*} \omega\right)_{x}-\omega_{x}}{t}=\left.\frac{d}{d t}\right|_{t=0}\left(\varphi_{t}^{*} \omega\right)_{x}
$$

Seja $X \in \mathfrak{X}(M)$ um campo vetorial suave e $\mathcal{L}_{X}: \Omega^{*}(M) \longrightarrow \Omega^{*}(M)$

Proposição A.2.2. Se $f$ é uma função suave em $M$ e $X$ um campo vetroial suave em $M$ então $\mathcal{L}_{X} f=X f$.

Demonstração. Se $\varphi$ é o fluxo de $X$, então

$$
\begin{aligned}
\mathcal{L}_{X} d \omega & =\left.\frac{d}{d t}\right|_{t=0}\left(\varphi_{t}^{*} f\right)_{x} \\
& =\left.\frac{d}{d t}\right|_{t=0}\left(f \circ \varphi_{t}\right)(x) \\
& =X_{x} f
\end{aligned}
$$


Proposição A.2.3. Seja $M$ uma variedade suave e $X \in \mathfrak{X}(M)$ e $\omega, \tau \in$ $\Omega^{*}(M)$. Então

$$
\mathcal{L}_{X}(\omega \wedge \tau)=\left(\mathcal{L}_{X} \omega\right) \wedge \tau+\omega \wedge\left(\mathcal{L}_{X} \tau\right)
$$

Demonstração. Seja $x \in M$.

$$
\begin{aligned}
\left(\mathcal{L}_{X}(\omega \wedge \tau)\right)_{x} & =\left.\frac{d}{d t}\right|_{t=0}\left(\varphi_{t}^{*}(\omega \wedge \tau)\right) \\
& =\left.\frac{d}{d t}\right|_{t=0}\left(\varphi_{t}^{*} \omega\right)_{x} \wedge\left(\varphi_{t}^{*} \tau\right)_{x} \\
& =\left.\frac{d}{d t}\right|_{t=0}\left(\varphi_{t}^{*} \omega\right)_{x} \wedge \tau_{x}+\left.\omega_{x} \wedge \frac{d}{d t}\right|_{t=0}\left(\varphi_{t}^{*} \tau\right)_{x} \\
& =\left(\mathcal{L}_{X} \omega\right)_{p} \wedge \tau_{x}+\omega_{p} \wedge\left(\mathcal{L}_{X} \tau\right)_{x}
\end{aligned}
$$

Proposição A.2.4. A derivada de Lie comuta com d: se $X$ é um campo vetorial suave e $\omega$ uma diferencial suave, então

$$
\mathcal{L}_{X}(d \omega)=d\left(\mathcal{L}_{X} \omega\right)
$$

Demonstração.

$$
\begin{aligned}
\mathcal{L}_{X} d \omega & =\left.\frac{d}{d t}\right|_{t=0} \varphi_{t}^{*} d \omega \\
& =\left.\frac{d}{d t}\right|_{t=0} d \varphi_{t}^{*} \omega \\
& =d\left(\left.\frac{d}{d t}\right|_{t=0} \varphi_{t}^{*} \omega\right) \\
& =d \mathcal{L}_{X} \omega
\end{aligned}
$$

Proposição A.2.5 (Fórmula mágica de Cartan).

$$
\mathcal{L}_{X}=d i_{X}+i_{X} d
$$

onde d é a derivada exterior.

Demonstração. A verificação do resultado é local, de modo a assumir que há uma carta em torno de certo ponto $x \in M$ para a qual as coordenadas 
são $x^{1}, \ldots, x^{n}$. Assim, podemos assumir $\omega=f d x^{i_{1}} \wedge \ldots \wedge d x^{i_{k}}$.

Ambos os membros das fórmulas são derivações que comutam com $d$, de modo que a validade dela para duas forma $\omega$ e $\tau$ garante a validade para $d \omega$ e $\omega \wedge \tau$. Isso implica que a verificação daquela fórmula faz-se checando que $\mathcal{L}_{X} f=\left(d i_{X}+i_{X} d\right) f$, para $f$ uma função suave no domínio da carta. Mas

$$
\begin{aligned}
\left(d i_{X}+i_{X} d\right) f & =d i_{X} f+i_{X} d f \\
& =d\left(i_{X} f\right)+i_{X} d f \\
& =d(0)+d f(X) \\
& =X f \\
& =\mathcal{L}_{X} f
\end{aligned}
$$

\section{A.3 Conexão de Levi-Civita}

(Baseado em [Tu17])

Definição A.3.1. Uma conexão $\nabla$ em uma variedade riemanniana $(M, g)$ é livre de torção se sua torção é nula, ou seja,

$$
\nabla_{X} Y-\nabla_{Y} X=[X, Y]
$$

para $X, Y \in \mathfrak{X}(M)$.

Definição A.3.2. Uma conexão é compatível com a métrica se

$$
Z g(X, Y)=g\left(\nabla_{Z} X, Y\right)+g\left(X, \nabla_{Z} Y\right)
$$

para qualquer $X, Y, Z \in \mathfrak{X}(M)$

Lema A.3.1. Um campo vetorial suave $X$ em uma variedade riemanniana $(M, g)$ é unicamente determinado pelos valores $g(X, Z)$, para todo $Z$ campo vetorial em $M$.

Demonstração. Devemos mostrar que se $g(X, Z)=g\left(X^{\prime}, Z\right)$ para todo $Z \in$ $\mathfrak{X}(M)$ então $X=X^{\prime}$. Isso equivale a provar que se $g(X, Z)=0$ para todo $Z \in \mathfrak{X}(M)$ então $X=0$. 
Vamos tomar $Z=X$. Então

$$
g(X, X)=0 \Rightarrow g_{x}\left(X_{x}, X_{x}\right)=0, \forall x \in M \Rightarrow X_{x}=0, \forall x \in M \Rightarrow X=0
$$

Teorema A.3.1. Se $(M, g)$ uma variedade riemanniana, então existe uma única conexão $\nabla: \mathfrak{X}(M) \times \mathfrak{X}(M) \longrightarrow \mathfrak{X}(M)$ livre de torção e compativel com a métrica $g$.

Demonstração. Pela compatibilidade temos

$$
\begin{aligned}
& X g(Y, Z)=g\left(\nabla_{X} Y, Z\right)+g\left(Y, \nabla_{X} Z\right) \\
& Y g(Z, X)=g\left(\nabla_{Y} Z, X\right)+g\left(Z, \nabla_{Y} X\right) \\
& Z g(X, Y)=g\left(\nabla_{Z} X, Y\right)+g\left(X, \nabla_{Z} Y\right)
\end{aligned}
$$

Das equações anteriores, vamos somar as duas primeiras e subtrair a terceira. Vamos combinar os termos usando também a condição de livre torção:

$$
\begin{aligned}
\nabla_{X} T \nabla_{Y} X & =[X, Y] \\
\nabla_{Y} Z \nabla_{Z} Y & =[Y, Z] \\
\nabla_{Z} X \nabla_{X} Z & =[Z, X]
\end{aligned}
$$

Obtemos assim

$$
\begin{aligned}
2 g\left(\nabla_{X} Y, Z\right) & =X g(Y, Z)+Y g(Z, X)-Z g(X, Y) \\
& +g(Z,[X, Y])+g(Y,[Z, X])+g(X,[Z, Y])
\end{aligned}
$$

Pela a fórmula acima para definir a conexão, pelo lema anterior, temos a unicidade os valores de $\nabla_{X} Y$ e, portanto, da conexão.

Vamos provar a existência mostrando que a conexão definida pela mesma forma tem as propriedade requeridas. Vamos chamar o lado direita dela de $\zeta(X, Y, Z)$. 
A função $\zeta$ é $C^{\infty}$-linear em $X$ e $Y$ :

$$
\begin{aligned}
\zeta(f X, Y, h Z) & =f X g(Y, Z)+Y f h g(Z, X)-h Z g(X, Y) \\
& +h g(Z,[f X, Y])+g(Y,[h Z, f X])+f g(X,[h Z, Y]) \\
& =f h X g(Y, Z)+f h Y g(Z, X)-f h Z g(X, Y) \\
& +f(X h) g(X, Y)+f Y(h) g(Z, X)+h Y(f) g(Z, X)-h Z(f) g(X, Y) \\
& +h f g(Z,[X, Y])+h f g(Y,[Z, X])+f h g(X,[Z, Y]) \\
& -h Y(f) g(Z, X)-f X(h) g(Y, Z)+h Z(f) g(Y, X)-f Y(h) g(X, Z) \\
& =f h \zeta(X, Y, Z)
\end{aligned}
$$

Além disso,

$$
\begin{aligned}
g\left(\nabla_{X}(f Y), Z\right) & =f g\left(\nabla_{X} Y, Z\right)+X(f) g(X, Y) \\
& =g\left(f \nabla_{X}+X(f) Y, Z\right)
\end{aligned}
$$

de maneira que $\nabla_{X}(f Y)=f \nabla_{X}+X(f) Y$

Definição A.3.3. A conexcõ acima definida no teorema anterior é chamada de conexão riemanniana ou conexão de Levi-Civita .

\section{A.4 Lema ou Truque de Moser Local}

Lema A.4.1 (Lema ou Truque de Moser Local). Seja $\left(\omega_{t}\right)_{t \in[0,1]}$ uma família de formas simpléticas diferenciáveis em $t$. Então, para $x \in M$, existe $U$ um aberto em torno de $x$ e $g_{t}: U \longrightarrow U$ tal que $g_{0}^{*}=i d$ e $g_{t}^{*} \omega_{t}=\omega_{0}$.

Demonstração. (Baseado em [Les14])

Vamos procurar uma família $X_{t}$ de campos vetoriais em $U$ de modo que

$$
\frac{d}{d t} g_{t}(p)=X_{t}\left(g_{t}(p)\right), g_{0}(p)=p
$$


Usando a fórmula mágica de Cartan na derivação de $g_{t}^{*} \omega_{t}=\omega_{0}$, obtemos

$$
\begin{aligned}
\frac{d}{d t} g_{t}^{*} \omega_{t} & =g_{t}^{*}\left(\frac{d}{d t} \omega_{t}+\mathcal{L}_{X_{t}} \omega_{t}\right) \\
& =g_{t}^{*}\left(\frac{d}{d t} \omega_{t}+d i_{X_{t}} \omega_{t}\right)
\end{aligned}
$$

O lema de Poincaré nos diz que, localmente, toda forma fechada é exata. A forma $\omega_{t}$ é fechada: $d \frac{d}{d t} \omega_{t}=\frac{d}{d t} d \omega_{t}=0$. Assim Em torno de $x$ há um aberto para o qual existe $\eta_{t}$ tal que $\frac{d}{d t} \omega_{t}=\eta_{t}$. Portanto,

$$
\begin{aligned}
\frac{d}{d t} g_{t}^{*} \omega_{t} & =g_{t}^{*}\left(d \eta_{t}+d i_{X_{t}} \omega_{t}\right) \\
& =g_{t}^{*} d\left(\eta_{t}+i_{X_{t}} \omega_{t}\right)
\end{aligned}
$$

Devemos encontrar $g_{t}$ como no enunciado, de maneira que $\frac{d}{d t} g_{t}^{*} \omega_{t}=0$, ou seja

$$
\eta_{t}+i_{X_{t}} \omega_{t}
$$

Como $\omega_{t}$ é não degenerada, a equação anterior tem solução com respeito a $X_{t}$ e define $\left(g_{t}\right)_{t \in[0,1]}$. Se $\left(x_{1}, \ldots, x_{2 m}\right)$ são coordenadas locais para $M$ então

$$
\begin{gathered}
\eta_{t}=\sum_{k=1}^{2 m} \eta_{k}(t, x) d x_{k}, X_{t}=\sum_{k=1}^{2 m} X_{k}(t, x) \frac{\partial}{\partial x_{k}}, \\
i_{X_{t}} \omega_{t}=\sum_{l, k=1}^{2 m}\left(2 \omega_{k}^{l} X_{k}\right) d x_{l}, \omega_{t}=\sum_{l, k=1}^{2 m} \omega_{k}^{l}(t, x) d x_{k} \wedge d x_{l}
\end{gathered}
$$

e assim, o sistema de equações a ser resolvida é

$$
\eta_{t}(t, x)+2 \sum_{k=1}^{2 m} \omega_{k}^{l} X_{k}(t, x)=0 .
$$

$\omega_{t}$ é não degenerada. Logo, $\left[\omega_{k}(t, x)\right]$ é matriz não-degenerada e, portanto, o sistema anterior tem solução única que determina os campos $X_{t} \mathrm{e}$, consequentemente, $\left(g_{t}\right)$ como desejada. 


\section{A.5 Propriedade Universal de Maurer-Cartan}

Teorema A.5.1. Seja $\eta \in \Omega^{1}(E, \mathfrak{h})$, tal que $d \eta+\frac{1}{2}[\eta, \eta]=0$. Então existe $U \subseteq E$ vizinhança de $x$ tal que há uma única $f: U \longrightarrow H$ de modo que $f(x)=e_{H}$ e $f^{*} \omega_{M C}=\eta$, sendo $\omega_{M C}$ a forma de Maurer-Cartan.

Além disso, se dimE $=\operatorname{dim} H$ então $f$ é um difeomorfismo.

Demonstração. Definamos em $E \times H$ a forma $\Omega=p r_{E}^{*} \eta-p r_{E}^{*} \omega_{M C}$.

Seja $D \subseteq T(E \times H)$ onde

$D_{(x, g)}=\left\{(u, \alpha) \in T_{x} E \times T_{h} H: p r_{M}^{*} \eta(u)-p r_{M}^{*} \omega_{M C}(\alpha)=\left(p r_{M}^{*} \eta-p r_{M}^{*} \omega_{M C}\right)(u, \alpha)=0\right\}$.

Vamos provar que o posto de $D_{(x, h)}$ é constante. Consideremos a função $D_{(x, h)} \rightarrow T_{x} E,(u, \alpha) \mapsto u$. Esta é um isomorfismo cuja inversa é

$$
T_{x} E \longrightarrow D_{(x, h)}, u \mapsto\left(u, d_{e} R_{h}\left(\eta_{x}(u)\right)\right)
$$

Assim, posto $D_{(x, h)}=$ postoE

Vamos provar agora que $k e r \Omega$ é involutivo: sejam $\xi_{1}, \xi_{2} \in k e r \Omega$. Primeiro, observemos que

$$
\Omega=p r_{E}^{*} \eta-p r_{E}^{*} \omega_{M C} \Rightarrow p r_{E}^{*} \eta=\Omega+p r_{E}^{*} \omega_{M C}
$$

Então

$$
\begin{aligned}
d \Omega & =d \omega_{M C}-d \eta \\
& =\frac{1}{2}\left(\left[\omega_{M C}, \omega_{M C}\right]-[\eta, \eta]\right) \\
& =\frac{1}{2}\left(\left[\omega_{M C}, \omega_{M C}\right]-\left[\Omega+\omega_{M C}, \Omega+\omega_{M C}\right]\right) \\
& =-\frac{1}{2}\left([\Omega, \Omega]+\left[\Omega, \omega_{M C}\right]+\left[\omega_{M C}, \Omega\right]\right)
\end{aligned}
$$

Consequentemente, se $\Omega\left(\xi_{1}\right)=\Omega\left(\xi_{2}\right)=0$ então $d \Omega\left(\xi_{1}, \xi_{2}\right)=0$.

Vamos provar que se $\xi_{1}, \xi_{2} \in \Gamma(k e r \Omega)$ então $\left[\xi_{1}, \xi_{2}\right] \in \Gamma(k e r \Omega)$. Temos

$$
d \Omega\left(\xi_{1}, \xi_{2}\right)=\xi_{1} \Omega\left(\xi_{2}\right)-\xi_{2} \Omega\left(\xi_{1}\right)-\Omega\left(\left[\xi_{1}, \xi_{2}\right]\right)
$$

Assim

$$
\Omega\left(\left[\xi_{1}, \xi_{2}\right]\right)=-d \Omega\left(\xi_{1}, \xi_{2}\right)=0 .
$$


Próximo passo será mostrar que $D$ é transversal às fibras de $\pi_{E}$. Sabemos que $\pi_{E} \subseteq T_{x} E \times T_{h} H$ e $\operatorname{ker} d_{(x, h)} \pi_{M}=\left\{(0, \alpha): \alpha \in T_{g} H\right\}$ e

$$
D_{(x, h)}+\operatorname{ker} d_{(x, h)} \pi_{E}=T_{x} E \times T_{h} H
$$

A respeito das dimensões

$$
\operatorname{dim} D_{(x, h)}+\operatorname{dim} \operatorname{ker} d_{(x, h)} \pi_{E}-\operatorname{dim}\left(D_{(x, h)} \cap \operatorname{ker} d_{(x, h)} \pi_{E}\right) .
$$

Mas $D_{(x, h)} \cap \operatorname{ker} d_{(x, h)} \pi_{E}=\{0\}$ pois $(u, \alpha) \in D_{(x, h)} \cap \operatorname{ker} d_{(x, h)} \pi_{E}=\{0\}$ implica $u=0$ e daí, $\eta(u)=0=\omega_{M C}(\alpha)$ o que implica $\alpha=0$.

Desse modo, foi provada a transversalidade de $D$.

Seja $N \subseteq E \times H$. a folha de $D$ que passa por $(x, e)$. Como $N$ e transversal a $\pi_{E}^{-1}(x)$, então $N$ é localmente o gráfico de uma função $f: U \subseteq E \longrightarrow H$.

Finalmente, $f^{*} \omega_{M C}(u)=\omega_{M C}(d f(u))$. além disso, $(u, d f(u)) \in T N$ de modo que

$$
\Omega(u, d f(u))=0 \Rightarrow \eta(u)-\omega_{M C}=0 \Rightarrow \eta(u)=\omega_{M C}(d f(u)) .
$$

de modo que,

$$
f^{*} \omega_{M C}=\eta
$$

\section{A.6 Exemplo não-integrável: variedades nearly Kähler}

(Detalhes em [Gra70]).

Uma variedade nearly Kähler é uma estrutura quase Hermitiana (análogo da quase-Riemanniana em $\mathbb{C}$ ) dotada de uma estrutura quase complexa $J$, em que $\nabla_{X} J(X)=0$, para todo $X \in \mathfrak{X}(M)$, sendo $\nabla$ não necessariamente de Levi-Civita.

Uma variedade Kähler é uma variedade Riemanniana, simplética e complexa. Toda variedade desse tipo é nearly Kähler mas não vale a recíproca.

Exemplo mais famoso de uma variedade nearly Kähler é a esfera $S^{6}$ que não é Kähler. 


\section{A.7 $\quad G_{2}$-estruturas}

Sejam $\mathbb{O}$ a álgebra dos octônios, O grupo $\operatorname{Aut}(\mathbb{O})$ dos automorfismo dos octônios é denotado por $G_{2}$ e denominado um dos grupos de Lie excepcional (de menor dimensão). Seu posto ${ }^{1}$ é 2 e tem dimensão 14.

Se $M$ é uma variedade tal que $\operatorname{dim} M=7$, então uma $G_{2}$-estrutura é a redução do grupo estrutural do fibrado principal $M$ ao grupo $G_{2}$.

\footnotetext{
${ }^{1}$ menor cardinalidade de um gerador do grupo
} 


\section{Referências Bibliográficas}

[Bel] Florin Belgun. Guage thoery. https://www.math.unihamburg.de/home/belgun/Gauge4.pdf. 38

[Cra15] Marius Crainic. Mastermath course differential geometry 2015/2016. Utrecht University, Setembro 2015. 3, 41

[dA17] Pedro Mendes de Araújo. Notes on the integrability problem for g-structures. Personal Notes, 2017. 69

[Dem12] Jean-Pierre Demailly. Complex Analytic and Differential Geometry. Universitéde Grenoble I, 2012. 53

[Dup03] John Dupont. Fibre bundles and chern-weyl theory. Aarhus Universitet, August 2003. 21, 22

[Fer03] Rui Lojas Fernades. Lições de geometria dierencial. https://www.math.tecnico.ulisboa.pt/ rfern/GD/notas.pdf, 2003. 79,80

[Gra70] Alfred Gray. Nearly kähler manifolds. Journal of Differential Geometry, Volume 4(Número 3):283-309, 1970. 89

[Gui65] Victor Guillemin. The problem of integrability for g-structures. Transactions of The American Mathematical Society, 116:544-560, 1965. 2,69

[KN63] Soshichi Kobayashi e Katsumi Nomizu. Foundations of Differential Geometry, volume I. Interscience Publishers, 1963. 24

[Lee97] John M. Lee. Riemannian Manifolds: An Introductin to Curvature. Springer, 1997. 46, 80

[Lee13] John M. Lee. Introduction to Smooth Manifolds. Springer, segunda edição, 2013. 51

[Les14] A. Lesfari. Morse's lemma and teh darboux theorem. Universal Journal of Applied Mathematics, 2014. 54, 86 
[NN57] A. Newlander e L. Niremberg. Complex analytic coordinates in a almost complex manifolds. Annals of Mathematics, 65(3), 1957. 53

[Sha97] R. W. Sharpe. Differential Geometry. Springer, 1997. 65

[Tu10] Loring W. Tu. An Introduction to Manifolds. Springer, segunda edição, 2010. 80, 81

[Tu17] Loring W. Tu. Differential Geometry. Springer, 2017. 84 


\section{Índice Remissivo}

$G_{2}$-estruturas, 90

1-forma de conexão, 21

ação

livre, 79

própria, 79

carta adaptada, 42

conexão

de Levi-Civita, 86

em fibrado principal, 20

riemanniana, 86

compatível com a métrica, 84

compatível com uma G-estrutura, 55

matrix de, 30

conexões

em fibrados vetoriais, 28

em fibrados vetroiais, 38

contato de k-ésima ordem , 71

curva horizontal, 24

curvatura

em fibrados principais, 26

em fibrados vetoriais, 33

derivada de Lie, 82

de campos vetorias, 80,82

derivada exteriror, 35

espaço horizontal, 20

espaço vertical, 18

estrutura

quase simplética, 53

simplética, 53

estrutura quase complexa, 51
Fórmula mágica de Cartan, 83

fibração de Hopf, 9

fibrado associado, 9

fibrado referencial, 18

fibrado vetorial, 15

pullback, 16

folheção, 50

forma de Maurer-Cartan, 65

forma tautológica, 59

forma tautológica de ordem k, 73

forma volume, 48

formas

em fibrados associados, 37

funcões estruturais, 43

G-estrtura

uniformemente k-integrável, 77

G-estrutura, 41

integrável, 42

G-fibrado principal, 3

fibra, 4

morfismo de, 6

trivial, 4

trivializável, 6

grupo do do tipo finito

do tipo k, 78

grupos de comologia de Spencer, 71

isomorfismo

de G-estruturas, 42

k-ésimo prolongamento , 69

k-ésimo tensor de estrutura de E, 77

k-jato, 72 
levantamento, 24

levantamento horizontal, 20

métrica riemanniana, 44

orientação, 47

prolongamento

primeiro, 57

Propriedade Universal de Maurer-Cartan,

88

redução do grupo estrutural, 39

referencial, 17

fibrado vetorial, 16

seção

de fibrado principal, 7

ao longo de uma curva, 31

de fibrado vetroial, 15

paralela, 31

subgrupo de tipo finito, 78

tensor de Nijenhuis, 52

torção

em fibrados vetoriais, 34

espaço de, 58

G-estrutras, 61

intrínseca, 58

transporte paralelo

em fibrados vetoriais, 32

transporte pararlelo

em fibrados principais, 25

Truque de Moser Local, 86

variedade nearly Kähler, 89

variedade riemanniana, 44

vetores

horizontais, 20

verticais, 20 